\title{
Art and Design Practices as a Driver for Deformable Controls, Textures and Screen Interactions
}

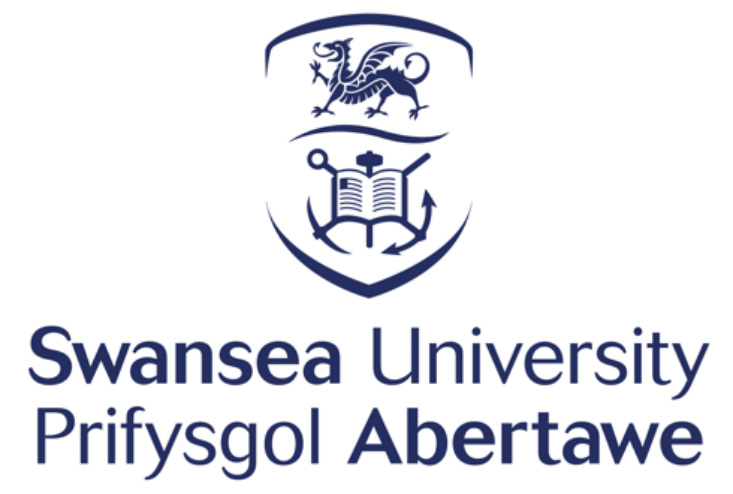

CAMERON STEER

Submitted in fulfilment of the requirements for the Degree of Doctor of Philosophy

Future Interaction Technology Laboratory

Department of Computer Science

College of Science

Swansea University 
To Mum \& Dad. My biggest supporters. 


\begin{abstract}
In this thesis, we demonstrate the innovative uses of deformable interfaces to help develop future digital art and design interactions. The great benefits of advancing digital art can often come at a cost of tactile feeling and physical expression, while traditional methods celebrate the diverse sets of physical tools and materials. We identified these sets of tools and materials to inform the development of new art and design interfaces that offer rich physical mediums for digital artist and designers. In order to bring forth these unique interactions, we draw on the latest advances in deformable interface technology. Therefore, our research contributes a set of understandings about how deformable interfaces can be harnessed for art and design interfaces. We identify and discuss the following contributions: insights into tangible and digital practices of artists and designers; prototypes to probe the benefits and possibilities of deformable displays and materials in support of digital-physical art and design, user-centred evaluations of these prototypes to inform future developments, and broader insights into the deformable interface research.

Each chapter of this thesis investigates a specific element of art and design, alongside an aspect of deformable interfaces resulting in a new prototype. We begin the thesis by studying the use of physical actuation to simulate artist tools in deformable surfaces. In this chapter, our evaluations highlight the merits of improved user experiences and insights into eyes-free interactions. We then turn to explore deformable textures. Driven by the tactile feeling of mixing paints, we present a gel-based interface that is capable of simulating the feeling of paints on the back of mobile devices. Our evaluations showed how artists endorsed the interactions and held potential for digital oil painting.

Our final chapter presents research conducted with digital designers. We explore their colour picking processes and developed a digital version of physical swatches using a modular screen system. This use of tangible proxies in digital-based processes brought a level of playfulness and held potential to support collaborative workflows across disciplines. To conclude, we share how our outcomes from these studies could help shape the broader space of art and design interactions and deformable interface research. We suggest future work and directions based on our findings.
\end{abstract}




\section{Acknowledgements}

Firstly, I would like to express my sincere gratitude to my advisor Matt Jones for the continuous support of my PhD study and related endeavours, and for his motivation, knowledge and support. Besides my advisor, I would like to thank the rest of my co-supervisors, Simon Robinson and Jen Pearson for their insightful comments and encouragement. Their guidance helped me in all my research and writing my thesis. In addition to my supervisors at Swansea University, thank you to Phil Stenson from BBC R\&D for extra support, access to BBC resources and unique perspectives from outside the university. Plus, thank you to Andrés Lucero at Alto University, who since meeting at the MoblieHCI DC, has been a great mentor whose expertise of working artists and designers helped guide my research.

I thank my fellow researchers and friends at Swansea University (past and present), during my $\mathrm{PhD}$ you helped make it an enjoyable experience, especially Tim Neate, Alix Goguey, Deepak Sahoo, Gavin Bailey, Stephen Linsey, Thomas Reitmaier, Tashi Gyaltsen, Chi Zang, Yogesh Meena, Joss Whittle, Richard Roberts, Jean Razafindrakoto and Dean Thomas. Thank you for the mix of professional support and recreational distractions.

In terms of financial support, my $\mathrm{PhD}$ work was fully funded by the College of Science at Swansea University, plus support from EPSRC grants and BBC R\&D, who provided additional funds, all of which I am very thankful for. I thank BBC $\mathrm{R} \& \mathrm{D}$ further for hosting me to run workshops and user studies. In particular, Rob Scott for his assistance in making this happen. Furthermore, I would like to thank all the participants that took part in my various research. In particular, Murton Art Class, Waters Creative and BBC UX\&D. Your time is greatly appreciated.

Behind the seances, thank you to my sibling Owain and their partner Rita for the fun down times from Bath to Helskini, and to my partner, Katie - you didn't know what you signed up for meeting me at the start of my $\mathrm{PhD}$, but you have always 
been there to support me. Finally, the biggest thank you to my parents, Mark and Tracey. The main source of all my support, emotionally (and financially). You both helped get me to this point and continue to be my biggest supporters, in all of my endeavours. 



\section{Contents}

Abstract

Acknowledgements $\quad$ ii

Declaration $\quad$ xvi

1 Introduction 1

1.1 Digital Art and Design . . . . . . . . . . . . . 2

1.2 Innovation using Deformable Interfaces . . . . . . . . . . 5

1.3 Methodology .................... 7

1.4 Contributions and Chapter Overviews . . . . . . . . . . . . 11

1.5 The Author's Contribution . . . . . . . . . . . . 15

2 Related Work 17

2.1 Tangible User Interfaces . . . . . . . . . . . . . . . . . . 19

2.1.1 Tangible Augmented Mobile Devices . . . . . . . . . . . . 19

2.2 Tangible Tools for Digital Art and Design . . . . . . . . . . 20

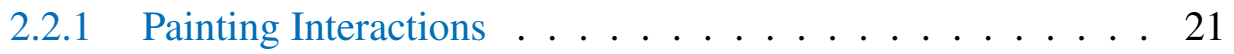

2.2.2 Stylus Interactions . . . . . . . . . . . . 21

2.2.3 Graphic Design Interfaces . . . . . . . . . . . . . 22

2.3 Working with Artists and Designers . . . . . . . . . . 23

2.4 Deformable Interfaces . . . . . . . . . . . . . . . . . 24

2.4.1 Displays and Controls . . . . . . . . . . . . . 25

2.4.2 Deformable Haptic Feedback . . . . . . . . . . . . . . 29 
2.4.3 Flexible Deformable Interfaces . . . . . . . . . . . . . 31

2.4.4 Dynamic Textures . . . . . . . . . . . . . 32

2.4.5 Modular Deformable Interfaces . . . . . . . . . . . . 33

2.4.6 Classifying Interactions and Actuation . . . . . . . . . . 34

2.4.7 Force-Based Interactions . . . . . . . . . . . . . . 34

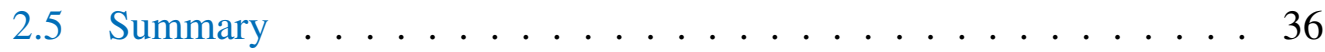

3 Actuated Art Tools 37

3.1 Introduction . . . . . . . . . . . . . . . . 38

3.2 Deformable Controls Driven By Art Scenarios . . . . . . . . . . . . 40

3.2.1 Prototype Design and Development . . . . . . . . . . . 42

3.2.2 User Study . . . . . . . . . . . . . . . . . . . . . 47

3.2 .3 Findings $\ldots \ldots \ldots \ldots \ldots \ldots$

3.2 .4 Discussion ..................... 55

3.3 Limitations . . . . . . . . . . . . . . . 57

$3.3 .1 \quad$ Next Steps . . . . . . . . . . . . . . . 58

3.4 Actuated Controls for Concurrent Shape-Change . . . . . . . . . 60

3.4.1 Shape-Changing Slider Cursor . . . . . . . . . . . . 61

3.4.2 Prototype Design and Development . . . . . . . . . . 62

3.4.3 Initial pilot study . . . . . . . . . . . . . . . 64

3.4 .4 Findings $\ldots \ldots \ldots \ldots \ldots 6 \ldots$

3.4.5 Discussion . . . . . . . . . . . . . . 69

3.4.6 Comparison to different output modalities . . . . . . . . 69

3.4 .7 Procedure . . . . . . . . . . . . . 73

3.4 .8 Findings . . . . . . . . . . . . . . 74

3.4 .9 Discussion .................. 80

3.5 Future Sensels for Art Tools . . . . . . . . . . . . . . . . 82

3.6 Summary ......................... 83

3.7 Authors Contributions . . . . . . . . . . . . . . . 84 
4 Dynamic Paint Textures $\quad \mathbf{8 5}$

4.1 Introduction . . . . . . . . . . . . . . . . 887

4.2 Observing Artists . . . . . . . . . . . . . 88

4.2 .1 Method .................... 88

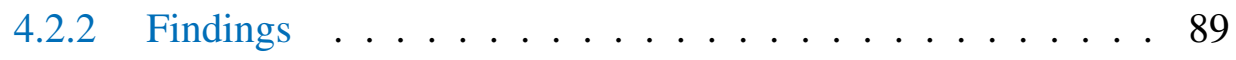

4.2 .3 Discussion ........................ 94

4.3 Prototype Design and Development . . . . . . . . . . . . 96

4.3.1 Hydrogel Paint . . . . . . . . . . . . . . . 97

4.3.2 Combining with a Mobile Phone . . . . . . . . . . 100

4.3 .3 Final Interaction . . . . . . . . . . . . . 100

4.4 Artist's Impressions of the Prototype . . . . . . . . . . . . 102

4.4 .1 Findings . . . . . . . . . . . . . 104

4.5 Evaluating the Gel States . . . . . . . . . . . . . . . 105

4.5.1 Method ..................... 106

4.5 .2 Results ....................... 108

4.5.3 Discussions and Limitations . . . . . . . . . . . . 112

4.6 Summary . . . . . . . . . . . . . . . . 115

4.7 Authors Contributions . . . . . . . . . . . 116

5 Modular Colour-Swatch Screens 117

5.1 Introduction . . . . . . . . . . . . . . 118

5.2 User Research Workshops . . . . . . . . . . . . . . . . . 120

5.2.1 Tangible Probes . . . . . . . . . . . . . . 121

5.2.2 Creative Design Agency . . . . . . . . . . . . 121

5.2.3 Broadcaster Designers . . . . . . . . . . . . . . 127

5.3 Workshops Discussion . . . . . . . . . . . . . . . . 129

5.4 Design Framework . . . . . . . . . . . . . . . 130

5.5 DigiSwatch Development . . . . . . . . . . . . . 132

5.5.1 Hardware and Architecture . . . . . . . . . . . 133 
5.5 .2 Software and Interactions $\ldots \ldots \ldots . \ldots . \ldots 135$

5.6 Feedback Sessions . . . . . . . . . . . . . . . . . . . 138

5.6.1 User Feedback . . . . . . . . . . . . . . . . . . 139

5.7 Discussion and Limitations . . . . . . . . . . . . . . . . . . . 142

5.8 Summary . . . . . . . . . . . . . . . . . 145

5.9 Authors Contributions . . . . . . . . . . . . . . 146

6 Discussions, Conclusion and Future Work 147

6.1 Summary of Contributions . . . . . . . . . . . . . . . . . 148

6.2 Discussion . . . . . . . . . . . . . . . 151

6.2.1 Chosen Context . . . . . . . . . . . . . . . . . 151

6.2.2 Future Art and Design Interfaces . . . . . . . . . . . . 152

6.2.3 Implications for Deformable Interfaces . . . . . . . . . . 155

6.3 Limitations and Future Work . . . . . . . . . . . . . . . 158

6.4 Concluding Remarks . . . . . . . . . . . . . . . . . 162

$\begin{array}{ll}\text { A Contributing Publications } & 165\end{array}$

$\begin{array}{ll}\text { Bibliography } & 168\end{array}$ 


\section{List of Tables}

3.1 Percentage of participants using physical and digital painting, and the frequency they use them on daily, weekly, monthly and yearly bases. ......................... 50

3.2 Average Time $($ Seconds $) \ldots \ldots . \ldots 51$

3.3 Average Error Ratings $(\%)$. . . . . . . . . . . . . . . 52

3.4 Median Likert ratings (1 low, 7 high) from matching task. Significant results marked with $*$ (Wilcoxon). . . . . . . . . . 52

3.5 Median Likert ratings (7 high) from the freeform painting task. Significant results marked with $*$ (Wilcoxon). . . . . . . . . . 53

3.6 Error Rating of Selections $(\%) \ldots \ldots 66$

3.7 Table shows the number of switches and time taken for the participants to select the boxes. . . . . . . . . . . . . . . 67

3.8 This table shows the error ratings, the number of switches and the time taken for the graphs to be drawn by the participants. . . . . . 67

3.9 This table shows the mean rating from the questionnaire given to the users. . . . . . . . . . . . . . . . . . 68

3.10 This table shows the average times and the number of switches for each output mode, including the Standard Deviation (SD) for each

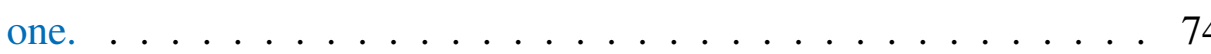

3.11 This table shows the percentage error averages and Standard Deviation (SD) of each mode for the overall sections and each small and large section . . . . . . . . . . . . . . . . 77 
3.12 It shows the statements the participants were asked to rate on a 7 point Likert scale (Low (1) up to High (7)). Groups with statistically significant effects are denoted with an asterix $(p<0.05(*)$ $p<0.005(* *))$. Shape Change (S.C.) . . . . . . . . . 78

3.13 This table shows the mean values of the users rating for the output mode (1-7) and the mean ranks positions $1^{\text {st }}$ through to $4^{\text {th }}$. Adjacent is each Standard Deviation (SD) value. . . . . . . . . . . 78

4.1 Frequency with which the participants used different painting tools . 107

4.2 Percentage of participants who labelled each material as either oil or acrylic . . . . . . . . . . . . . . . . 109

4.3 Median confidence rating participants labelling in task 1. We show the Median confidence of all the labelling together in under overall. Then the confidence of all correct labelling and incorrect labelling. $\mathrm{SD}=$ Standard deviation $\ldots \ldots \ldots \ldots \ldots \ldots$ 


\section{List of Figures}

2.1 This shows key areas of related work we consider relevant to the work presented in the thesis. The white circles highlight the areas in which the prototypes sit. Our focus is on the intersection of art interfaces and deformable interfaces. Some of the work also overlaps with mobile HCI due to the nature of the prototypes $\ldots \ldots 18$

3.1 Sketch illustrates a user with a deformable mobile and second screen canvas. Here we see the user approach the canvas screen with a deformable devise (Top-Left). The canvas activates (Top-Right). When the user needs to select a brush type and size the sensels emerge to form the brushes (Bottom-left). Then the user needs to select a colour and saturation. So the sensels submerge to form the paint pots (Bottom-Right) . . . . . . . . . . . . . 41

3.2 These images show the sensels built for the prototypes before they were attached the actuator. We positioned five force sensors on each face of the sensel (Left). Then we encased the sensel in foam (Right). . . . . . . . . . . . . . . 42

3.3 These diagram shows a cross section of the string and pulley system used the create the actuation. . . . . . . . . . . . . . . . 43

3.4 This diagram illustrates the colour picking state of the sensel along with force sensing directions. When the sensels submerge it forms a paint pot for paint selection. Here, the force acts down onto the foam and forms the push gesture. . . . . . . . . . . . . . 44 
3.5 This diagram illustrates the nib picking state of the sensel along with force sensing directions. When the sensel emerges it forms the nib for nib resizing. Here the force acts upon the sides of the foam and forms the squeeze gesture. . . . . . . . . . . . . . . 44

3.6 This figure shows prototype being used the custom built software. . 46

3.7 This screen shows the interface participants used on the touch screen. Its made up of three sliders positions and sized the same as the sensels on the deformable . . . . . . . . . . . . . .

3.8 Study-up: Participant using the GUI tablet (left) Participant using the deformable(right) . . . . . . . . . . . . . . . . . 48

3.9 Study Modes: Nib Matching (top-left) Paint Matching (top-right) Painting mode (bottom) . . . . . . . . . . . . . . . 49

3.10 Number of participants using physical and digital painting, and the frequency they use them on daily, weekly, monthly and yearly bases. 51

3.11 Deformable slider implementation. . . . . . . . . . . . 63

3.12 Diagrams illustrating three possible stages of deformation: Fully open indicates the largest value; in our software this was 10 (Top), Mid stage open; in our software this was 5 (centre), fully closed, indicating the smallest value; in our software this was 0 (Bottom) . .

3.13 Screenshot of the box selection task. Here, the slider is positioned

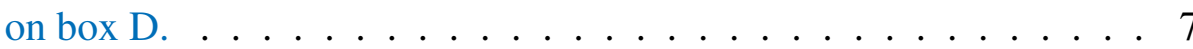

3.14 This figure shows a labelled diagram of the comparison study's setups from the perspective of how the user sits to the deck to use the

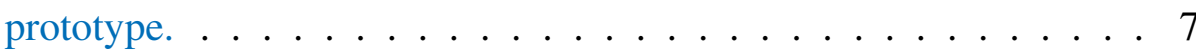

3.15 The percentage of incorrect guesses for each slider mode for the user selections. Top shows the overall percentages. Bottom left shows only guesses selecting the largest values. Bottom right shows only guesses selecting the smallest values. Error bars illustrate the standard error in each data set. . . . . . . . . . . . . . 
3.16 The graphs here show the average number of switches (Left) and the average time (seconds) taken to make selections (Right) for each output mode. . . . . . . . . . . . . . . . 76

3.17 Returning to the figure from Section 3.2.1 we illustrate how actuation as shown in the slider study could be added to sensels as seen in the first prototype. . . . . . . . . . . . .

4.1 Painting Tools: Artist knifes (left), Artist Pens with interchangeable nib sizes (centre), Brushes: these three different brushes were being used simultaneously by and artist (right) . . . . . . . . . . . . . 90

4.2 Artist using painting knifes to create raised areas in painting. They do this by scooping up areas or layering on thicker amounts of paint. The image here show an oil painting. . . . . . . . . . . . . 91

4.3 Artist using palette for paint mixing before taking the paint to canvas. Notice how the palette colour matches the blends of colour on the canvas. The palette gives a history of the colours used in their

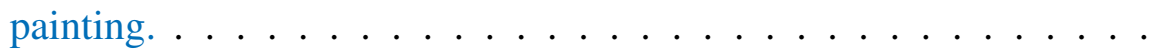

4.4 The mixed colours left on a palette after painting. This shows the history of the colours mixed for the painting . . . . . . . . . .

4.5 Left figure shows how digital artists will add an extra layer when they're painting in Photoshop for mixing paint. They will hide and reveal this layer as needed. Right figure shows the digital artist's work station. . . . . . . . . . . . . . .

4.6 Hydrogel states as temperature is changed, Left: The gel is at its hardest, feeling like an oil-based paint. Centre: The gel is softer and thinned out, feeling more like an acrylic-based paint. Right: The gel is now at its softest after being fully thinned out, and feels more like a water-based paint. 
4.7 This figure shows a transparent view of the prototype. Each Peltier module is set under the gel. The red Peltier module shows stiff gel in a fully white state and the blue Peltier shows soft gel in a transparent state. . . . . . . . . . . . . . . . 98

4.8 This figure illustrates the layers of the prototype used to activate the gel. Here, a Peltier module is set to cool the gel (blue side). . . . . . 99

4.9 The GUI displayed on the phone. On the left is the menu to select the initial colour. On the right, we see that colour is now in the centre pot, which activates the gel in that area on the back. . . . . 101

4.10 Left: Artist using palette and canvas. Right: Our digital prototype with tablet canvas application and mobile paint palette. . . . . . . 102

4.11 This figure shows the prototype as a whole from a side view, showing both the back gel screen and the mobile phone. . . . . . . . . 103

4.12 Number of participants that used different painting tools at daily, weekly, monthly, yearly and less frequency . . . . . . . . . . 108

4.13 Number of correct guesses for each paint, including the error bars. . 110

5.1 Siftio Cubes used in the workshops running the colour matching program. . . . . . . . . . . . . . . . 122

5.2 Images made for one of the interactive wireframes to compliment colours. Wireframes were made using Adobe XD . . . . . . . . . 123

5.3 Pantone®bridge kits used by designers with clients to determine colours for brands and resources. e.g. flyers, leaflets and posters. . 124

5.4 Workshop with the BBC UX\&D Designers . . . . . . . . . . 125

5.5 Diagram shows the full colour picking process for both groups of

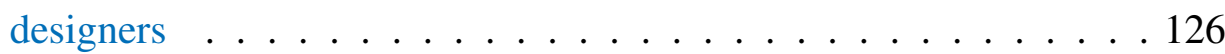

5.6 Overview of the steps a designer takes using existing tools based on

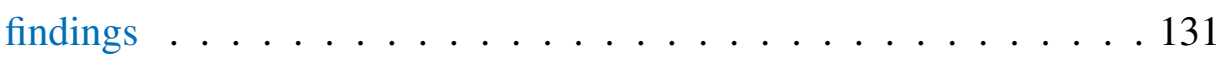


5.7 Our vision for how tangible tools can combine the elements for a more seamless colour picking process . . . . . . . . . . 133

5.8 DigiSwatch: Figure illustrates how the combining modular screens deliver colour discovery and manipulation in groups of connected

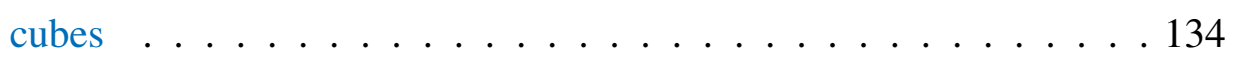

5.9 DigiSwatch (left) Software (right). The software shows the current configerations of the cubes. It also allows for sampling colours. . . . 135

5.10 (a) Switching between different cube modes (default, master and contrast) via long press interactions. (b) Illustration of a colour picking layout. Adding cubes horizontally changes the lightness and vertically changes the hue. Each change is based off the master cube. (c) Contrast checker cube in the centre of two coloured cubes. Output is displayed based on both left and right adjacent cubes. . . . 136

5.11 Contrast checker cube with colours on the left and right sides. In the centre, a contrast value with a red background for not compliant with colour accessibility standards. . . . . . . . . . . . . 138 


\section{Declaration}

This work has not been previously accepted in substance for any degree and is not being concurrently submitted in candidature for any degree. The work in this thesis has, however, undergone peer review and has been presented at major international venues. The publications noted below were prepared for submission during my candidature. Details of the papers and author's contributions are documented in Appendix A.

(P1) Cameron Steer, Jennifer Pearson, Simon Robinson and Matt Jones. Deformable Paint Palette: Actuated Force Controls for Digital Painting. CHI 2017 Works-in-Progress, Denver, May 2017

(P2) Cameron Steer Designing Mobile Deformable Controls for Creation of Digital Art. Mobile HCI 2017 Doctoral Consortium, Vienna, September, 2017

(P3) Cameron Steer, Simon Robinson, Jennifer Pearson, Deepak Sahoo, Ian Mabbett, Matt Jones. A Liquid Tangible Display for Mobile Colour Mixing. In Proceedings of Mobile HCI 2018, Barcelona, September 2018.

(P4) Alix Goguey, Cameron Steer, Andres Lucero, Laurence Nigay, Deepak Sahoo, Celine Coutrix, Anne Roudaut et al. PickCells: A Physically Reconfigurable Cell-composed Touchscreen. CHI 2019. ACM.

(P5) Cameron Steer, Alix Goguey, Andres Lucero, Simon Robinson, Jennifer Pearson, Matt Jones, and Phil Stenton. DigiSwatch: A Tangible Modular Tool for Designers to Interact with Digital Colours. Under Peer Review for ISS 2019. 
Statement 1 - A declaration that the work has not previously been accepted in substance for any degree and is not being concurrently submitted in candidature for any degree.

Statement 2 - A statement that the thesis is the result of your own investigations, except where otherwise stated and that other sources are acknowledged by footnotes giving explicit references and that a bibliography is appended.

Statement 3 - A statement that the student gives consent for the thesis, if accepted to be made available online in the University's Open Access Repository and for inter-library loan, and for the title and summary to be made available to outside organisations. 


\section{Chapter 1}

\section{Introduction}

Interfaces for digital art and design are continuously evolving with new and updated tools and materials being incorporated into the work of artists and designers. In this thesis, we considered a potential future for digital art interfaces by harnessing new deformable interaction technologies to develop richer physical experiences. During this century, we have seen the emergence of digital tools and how they provide benefits such as accuracy, recovery from mistakes, the use of algorithms and the easy duplication of work. Furthermore, it's increasingly accessible and portable, so an artist or designer may take a complete studio of digital tools, in a single device, wherever they go. This innovation offers creatives new possibilities for what they can make and has given digital art traction, making it a viable medium

alongside other creative tools. Given such benefits and traction, our vision for its future lies in enhancing the physical richness of these digital interfaces, informed by the understandings of traditional methods and the demonstration of deformable prototypes.

Like its traditional counterparts, the digital tool is the pathway between the artists and their work, and while digital art is advanced, it is still not equal in physical richness when compared to aspects of traditional methods. In tackling this contrast, in our research we explored key areas of physical art such as the brushes 
for painting, the paints used to mix colours, and the swatches ${ }^{1}$ and mood boards[67] used to support colour picking. From these understandings, we developed a set of prototypes that employ aspects of these physical processes and merge them with digital elements. These prototypes build upon deformable interface research such as the use of physical actuation to represent tools, dynamic textures that feel like paints, and modular devices for digital swatches. By harnessing these technologies and working with artists and designers, we demonstrate how to create new hands-on experiences where the tangibility makes for a more playful and creative experience. This thesis presents these prototypes with evaluations to demonstrate the potential future they hold for enriching interactions with digital art and design interfaces.

\subsection{Digital Art and Design}

As outlined the research in this thesis explores digital art interfaces or the purpose of this thesis when we refer to "Digital Art" as a broad concept we refer to the creation of digital drawing, illustration and graphic design in software such as Photoshop and Illustrator. This chosen area had an array of digital interaction to explore in our research we many of them simulating what would have traditionally been done with a variety of physical tools such as paints, pencils or pens. Early sections of the thesis are focused on the experiences using these tools considering the difference between the digital and physical while the remainder dives deeper into aspects of colouring mixing and picking within the digital drawing, illustration and graphic design.

We see in the history of digital art the theme of using physical practices to inform digital innovations. Innovations in digital art had been taking place since the early 1960s, where we first saw the advent of digital art tools as computer technology be-

\footnotetext{
${ }^{1}$ We refer to swatches as samples of colour whether physical (e.g. Pantone ${ }^{\circledR}$ books) or digital (e.g. colour squares in Photoshop ${ }^{\odot}$ ).
} 
came powerful enough to support its input and outputs. The earliest form of graphical manipulation came from Ivan Sutherland with the creation of Sketchpad[116]. This example was the first demonstration of computer graphics for both artistic and technical purposes, and for the first time, it gave users control over the graphics on the screen. It supported drawing with a light pen and had various side buttons to allow for erasing and moving objects. Compared to modern day devices, it had limited capabilities but it set the stage for others to innovate as new technologies advanced and took the field to new directions both academically and commercially.

Early research from Ivan Sutherland lead to the first commercial digital paint product, MacPaint ${ }^{2}$, which was released in 1984 . MacPaint was advanced because it had GUI-based tools, which were similar to real-world tools such as pens, shapes and swatches. It also used a mouse to bridge the gap between the artists and their art in these new digital worlds. Leading on from MacPaint, the image manipulation software Photoshop ${ }^{3}$, widely considered as the industry standard, was first conceived in 1988. Current versions of Photoshop provide digital artists with a complete art studio wherever they take their device, making digital creation portable and accessible. These tools come with primary features such as drawing and painting with a digital pen and brush tools, to more advanced examples, such as masking and gradient manipulations. All of these features emulate some existing art practice or new possibilities that only digital can provide.

Throughout these developments, we see the considerations for tangible experiences. Advanced stylus technologies have been researched and developed since 1968 with the RAND Tablet, and have continued with market-leaders Wacom; Apple and Microsoft. Tools like styluses, tablets and colour dials are the result of attempting to emulate real art and design techniques beyond the software layer. In fact, the vast majority of research that provides tangible elements for digital painting has focused on innovative stylus hardware for realistic paint and brush effects

\footnotetext{
${ }^{2}$ MacPaint: https://bit. Iy/2FDMVbn

${ }^{3}$ Photoshop: https://adobe. 1y/1JWYMCG
} 
in graphical software $[133,132]$. The most recent advancements include a pen that can be shaped by users [66]. These simulation attempts have led to advanced tools which are used by many digital artists. The endeavours also show a continuing search for richer tangible experiences for digital art and an interest in simulating the physical aspects of traditional methods.

As digital methods reach their maturity, the development of creative support tools has become a growing area of research. It includes the research into designing, building and testing tools that harness emerging technologies that deepen our understanding of creative practices with digital technologies [108]. Part of these advancements is the ongoing development of tangible tools that aim to provide hands-on experiences that emulate some of the richness of traditional methods. We saw this advancement as an opportunity to explore how artists and designers use digital and physical tools, and to then develop new technologies that demonstrate new directions that are not necessarily directly related to styluses.

When we consider traditional mediums, we see that they are tangibly rich experiences with a great diversity of tools and materials, each accompanied by physical sensations. These experiences are ones that are physical and rooted in creative expression developed over the history of humankind, and for many artists, they play a role in their enjoyment of the medium. Tools such as brushes come in many different shapes and sizes, and are key for the artist to create the right stroke. Materials like paints provide viscous sensations of mixing and stirring to obtain that perfect shade or saturation. These considerations focused our observations on the tangible elements of art and finding aspects considered challenging to emulate with existing GUIs and TUIs. In this thesis, we gather an understanding of art and design practices through observations and engagements with artists and designers. These studies allowed us to consider how to utilise novel technologies in order to develop new richer interfaces for their practices not yet explored in the literature. 
In each chapter we explored critical aspects of art and design. First, we considered the different tools used and switched between, such as brushes and paint pots. We studied how these physical forms don't match up to the limitations of touchscreens or static shapes of styluses. Further exploration into the materials used highlighted the importance of textures and how painting affords gestures such as stirring and mixing. Finally, to gain a deeper understanding of colour picking, we observed the physical benefits that colour swatches bring to the colour picking process and the advantages of using physical space for exploring and viewing the colour.

These areas of art and design do not yet have rich tangible analogues for digital forms, potentially limiting the richness of interactions, and for some artists cause a missed physical connection between them and their work. Therefore, we used these areas to explore the use of the latest interaction technologies to build prototypes addressing these digital-physical divides. Illustrating how digital interfaces with richer interaction capabilities can be more akin to their physical counterparts.

\subsection{Innovation using Deformable Interfaces}

In order to innovate and move towards our vision of richer tangible experiences with digital art, we considered deformable interfaces as the next advancements in tangible interaction technologies. We use this technology to drive the prototypes made for this thesis based on the areas of art and design we observed. This thesis takes a broad definition for Deformable defining it as physical interfaces used to interact with the digital world (much like a tangible interface) but also has some type of ability to dynamically change whether it be changing the physical for output and/or input. By taking this broad approach it kept our design options open for the array of possible study art and design offers and avoided narrowing the scope of prototypes we could have built. 
In the literature of tangible user interfaces (TUI) we see many researchers drawn to the field of deformable interfaces as the next evolution in tangible media [42]. These interfaces aim to combine the benefits of TUIs and the dynamic elements of graphical user interfaces (GUIs). They achieve this benefit by physically changing, based on users' interactions and desired outputs, aiming to bring richer interface possibilities to human-computer interaction. Deformable interfaces contain dynamic shape-changing elements that combine the benefits of the dynamic change in GUIs, with the physical interaction benefits of TUIs. This benefit is often achieved through the use of actuation in three-dimensional space [21]. This kind of interaction brings a physicality to interfaces while maintaining the aspects of versatility associated with GUIs.

So far, work within the literature has shown exploration into possible future interface development, design spaces, and a motivation to develop hands-on interactions for users. The areas of deformable interfaces deployed and explored in this thesis are: form changing controls, dynamic textures and modular devices. We report on how they can serve art processing and detail their implementations. We explore what is possible with digital art interaction and demonstrate the new possibility for deformable interfaces.

First, we consider form-changing interfaces, that use physical actuation to change shape, to present some new control or input possibility. It is often used to portray a change in affordance or constraint. Changing the form of a physical interface also allows it to represent a real-world object. We explore this use, by demonstrating its applications for representing tools for painting controls and extending possible input controls.

We also draw on key work in dynamic textures as a medium to enrich the feeling of certain materials. Dynamic textures are used in systems to provide users with some feeling of texture for haptic sensation. The use of dynamic textures means that the feel of an interface is not limited to a single sensation, but can change 
programmatically, and it's therefore able to provide an array of textures. We deploy this use of textures in new ways, testing their ability to act as new sensations and afford the use of gestures during the state change.

Finally, we also bring in elements of modular devices. Research into deformable interfaces shows how the device of the future will be able to be broken apart and reconfigured $[22,100]$. This ability will allow for users to change functions based on shapes, and share information via physical exchanges of parts of devices. We use this future scoping concept to study how it can support browsing data in creative scenarios and gather an understanding of how professional creatives would use such devices in a scenario like colour picking.

These new forms of interactions are yet to be explored in the context of art and design. So in this thesis, we have drawn upon these key types of deformable interactions to develop richer digital art and design interfaces. We do this by taking advantage of key advancements in form-changing, dynamic textures, and modular interfaces. We identified the use of each one in our studies of art and design methods, making them an essential element of our research process.

\subsection{Methodology}

Given the benefits of digital art and the advanced tangible tools developed for art, our vision in this thesis was to consider how we can take the next step from tangible art interfaces to deformable art interfaces. In realising this vision, we made digital art tools more realistic by adding new deformable elements which combine the benefits from both graphical and physical interfaces. We do this by taking a usercentred design approach that provides us with clear research steps to engage with art and design processes, then building prototypes based on the latest deformable technology, before finally returning to the users for evaluations. Ultimately, this bridges the research gap between tangible art and design tools and deformable interfaces. 
In following this approach, we engaged with artists and designers, practising both traditional and digital methods to understand their workflows and how they use existing tools and materials that might inform the development of new interfaces. From these understandings, we built prototypes with aspects of real-world art and design tools combined with digital elements. Findings from studying the art and design via direct observations and discussions were used to inform the development of our prototypes, driving what new form factors of deformable technology can take shape. We then evaluated the new prototypes with users to gather insight into what benefit they might hold and what might inform future work.

In realising these methods, we used a mix prototyping, lab-based user studies, observational studies and workshops to gather insights into the world of art and design. Different chapters in this thesis take different orders to how they approach these methods. In Chapter 3 we used bottom-up were the prototype is developed based on existing deformable literature and shaped to the context of art and design. Doing so allowed us to find gaps in the literature presented by the HCI research community but limited upfront learning from users in the prototypes end use-cases scenario. Based on these limitations we took a different order of approach in Chapter 4 . Here we shift to a more top down focus, first taking to the users and understanding their exiting processes before then building a prototype based on the finding to then return and test in user studies. This approach gave us a deeper understanding of the context before we started to build the prototype for evaluation.

Then the final chapter approaches with a mix of the two methods. We took a halfdesigned prototype based on the ideas collected in the previous observations into workshops with the designers. Here we were able to learn from the designer about their existing process and use those finding to further shape the prototype, as done in Chapter 4. This approach gave the workshop participants time to use the early version of the prototype offering us early insights to it viability enabling more back and forth learning between the participants and researcher. It also gave the user the 
opportunity to give feedback with a basic understanding of the possibilities shape changing interfaces. This benefit could achieve as the designers were more digitally native due to the context of their work allowing them to give more constructive feedback.

In cases were we went to user first we placed ourselves in design studios and traditional art classes. There we observed artists at work, giving us a primary source of observations for the tools and techniques. The environment allowed us to see common processes alongside conducting interviews to understand accounts of personal experiences. This process allowed us to gather understandings and requirement that served to inform the develop our prototypes and conceptualise take-aways for future interaction designers. Overall, these observations provided us with a deeper understanding of the artist's world.

In some cases we used the workshop methods to generate ideas for new technology and to gather feedback on early-stage ideas and prototypes. Workshops would open with further opportunities to learn from designers about how they work. This time, we asked more probing questions about what could be done to improve and which technologies we could develop to help. We also took along the early stage wireframes and low fidelity prototypes, which provided us with opportunities to gather feedback for improvements and direction in their further development. We conducted the workshops with groups at their place of work. We thoroughly document the process in Chapter 5.

To receive this feedback, we conducted user studies with all the developed prototypes to evaluate their benefits according to our original observations. We conducted user studies where people engaged in tasks using the prototypes, and we collected data using HCI research methods, such as questionnaires, interviews, and data logging. We measured our success based on the user's experience, highlighting qualitative descriptions of their time spent with the prototypes. We also wanted to understand how well these new prototypes facilitated digital manipulations through 
tasks that emulate specific aspects of the traditional interactions. It allowed us to gauge how well the prototypes currently function and highlight future works needed to support the artist better. In each chapter, we outline study-specific measures.

In experiments where we tested our developed prototypes, we used interviews together with qualitative data and carried out thematic analysis. This data gave us additional opinions on how to develop devices and identify themes across participant feedback. In addition to the interviews, when conducting experiments with the prototypes, we logged data on the devices. This allowed us to capture interactions not easily observed or measured by the researcher. In our evaluations studies, we employed established user HCI research practices [6, 61]. These included recording observations, conducting interviews and questionnaires and holding observations and user workshops. We also collected device log information when using prototypes. Using these types of data collection methods, we gathered information about the work practices of artist and designers and identified areas for intervention. We used an ethnographic approach with interviews and observations. In our observations, we took notes and audio recordings, video and photography. We transcribed all observations and collected them together for thematic analysis.

We also use questionnaires in some experiments. These questions include Likerttype scales, rankings and ratings. Both the log data and questionnaires give us quantifiable data from the experiments. This data allows us to conduct statistical analysis so to report how users interact with the system and the subjective ratings and rankings with the degree of confidence. We provide a detailed account of each statistical test used in each of the chapters, which we report in the results sections.

To develop the deformable interfaces, we undertook extensive prototyping where we explored a variety of existing scientific and engineering techniques. We have chosen a deformable interface as an approach to innovation in the art and design context. This approach will allow us to draw on the human centred approaches 
seen in the past related work when building such interesting. There are other approaches to innovation in this space such as material driven where we focus innovation of new materials first and see how art and design. Another approach may have been engineering driven where we focus solely on the mechanical novelty things we could build. These would probably bring about more polished prototypes and interesting physical innovation but may sacrifice study of human computer interaction and loose focus on the artists. As building deformable interfaces with a high-fidelity of control is a significant engineering challenge, so for the most part, the prototypes presented in this thesis do not innovate in the field of engineering or industrial design. Instead, our focus is interaction design, and therefore the prototypes are presented as possible mockups of their intended interactions in order to receive user feedback and evaluation.

\subsection{Contributions and Chapter Overviews}

The research presented in this thesis is aimed primarily at three key audiences. Firstly, we aim to provide HCI researchers with deformable interfaces with examples of new physical materials and interaction methods based on our chosen context. Secondly, this research will inform audiences of HCI researchers that study novel interactions for art and design of physical elements could be harnessed and what mediums arise from. Then finally, this thesis targets designers and developers of digital art and design tools and peripherals outside academia. Those designing and developing products used with existing software in-order to provide inspiration and a glimpse into future possibilities for their industry.

Each of the chapters outlines a specific study exploring a particular element of the artistic experiences and contribute a new prototype and evaluation. For each element, we will describe the contributions of the studies concerning what we outlined above. Here is an overview of what each chapter contributes: 
In Chapter 2, we document the essential related work in the space of TUIs for art and design, tangibly augmented mobiles and deformable interfaces to identify the gap for our research. We begin by outlining other research within the space of developing interfaces for digital art. This review includes a discussion of specific stylus and canvas technologies, alongside other unique forms of physical interactions. We also discuss the methods of this type of work, highlighting how other researchers have conducted studies with artists and designers to inform their research. We then consider the area of deformable interfaces and discuss the different types of physical input and output devices and technical methods developed. This will touch on the key areas of physical actuation, dynamic textures and modular devices. To summarise, we outline the gaps between tangible art interfaces and the use of deformable interaction techniques from the presented literature. This gap created the opportunity for our research to enrich interaction digital art creation with deformable interfaces.

In Chapter 3, we first look at how we could emulate physical objects from traditional art methods. This chapter will look at physical actuation to change the form of an interface that can present different controls which transform to make physical art tools. We do this in a device where areas can submerge to create paint pots and also emerge to create nibs. In each case, users could stick their hands into submerged elements, such as pots, and push down to select paint or grab emerged elements to squeeze and shape their nibs. In a user study, we compared this system to sliders on a touchscreen tablet. We found that the tablet was significantly more accurate and faster when making selections, yet despite this, people enjoyed using this device and found it to be quite an expressive way of painting. This first study began to highlight the benefits of more engaging forms of tangible interactions and how they could benefit existing digital art applications for more playful interactions. Despite this benefit, it was still limited in the accuracy and speed it provided compared to GUI controls. Therefore, we wanted next to improve the elements that attempted to copy textures and improve the controls by making it more like stirring 
or dipping in the paint.

In Chapter 4, we explore the use of deformable interfaces for digital painting. Going further down this colour picking route, we talked to different painters, both traditional and digital. We observed how a lot of them liked hands-on elements of the traditional methods. They were able to mix colour and understand precisely how much they were dragging in how much they were scooping, providing a context to the experience. After identifying this element of the painting experience, we saw an opportunity to emulate paint textures using chemically synthesised materials for digital painting. We built a system using hydrogel that could change texture based on the temperature providing gestures such as stirring. We put this on a mobile device and paired it with a colour palette application. This pairing meant that users were able to feel the sensations of mixing paints on the back of the screen that was paired with visuals of paint spreading and mixing on the front screen. The chapter contributes details on the building and testing of a hydrogel prototype to simulate textures or paints, followed by a feedback session with artists and a lab-based study identifying the similarity of the gels to oil-based paints.

In Chapter 5, we extend our exploration of colour interaction by working with digital designers to understand their process of colour picking and mixing. We specifically worked with designers in marketing or graphic design and studied how they picked and gathered colours for their designs. We observed how they work with colour swatches and how teams of designers come together to pick colours and build guidelines for brands and products. We outline findings from user research conducted with a creative design agency and the BBC design team. From these sessions, we map out scenarios informed by current design workflows and highlight the limitations and problems they face with existing tools. Drawing on the visions of modular deformable devices, we report a design process with feedback on low-fi prototypes and open idea generation. Ideas centred around digitising physical colour swatches via small modular screens that can be moved, attached and 
edited to find colours. We outline the technical implementation of the final modular system named DigiSwatch and describe the results from user studies conducted with the designers. We present the implications it has on the workflow of designers about making new tools and interfaces, along with the broader implication of mobile screens that can be broken apart and modularised. The research demonstrates an aim to create new expressive hands-on experiences for digital artists and designers, using tangibility to make for more playful and creative experiences.

Considering all the research across all three chapters, we distill our contributions and insights into the following key takeaways:

1. A set of understandings gathered from artists and designers about the tangible experiences in art and design and how each of the digital and traditional method differ. These findings come from the key areas of tools such as brushes and paint pots and what their physical role is to an artist compared to tools in digital; we then surface the role of the texture of physical paints and gestures associated with mixing to make colour, which are not present in digital systems; and finally, we identify the use and constraints of physical colour swatches in the designer's picking of colours and the importance of tangible objects for colour exploration. These understandings inform the contributing prototypes.

2. A set of novel prototypes that demonstrate different elements of art and design and merge digital and traditional worlds. These prototypes harness new uses of shape-changing displays for physical tool representation and switching in a single interface, dynamic texture interaction using hydrogel to simulate feelings and gestures associated with mixing paints; and a modular device that can be broken apart for each screen to be used as a swatch, capable of shape configurations for a dynamic exploration of colours.

3. A set of evaluations for each of the developed interfaces with the original observations showing the performance and viability of the art and design tasks 
explored, along with implications for the future of digital art tools and how the current state of the art can apply these novel techniques in real scenarios.

\subsection{The Author's Contribution}

The vast majority of this research was undertaken by the thesis author, who took the role of HCI researcher, interaction designer and hardware developer. All user research and requirement gathering was done solely by the author with the resulting design and concept of each prototype discussed with a supervisory team. The majority of the development for each prototype was then carried out by the author, who received support from Swansea Chemistry Department to source materials and demonstrate how to safely prepare hydrogel. The construction of DigiSwatch was supported by a fellow researcher, who used it for a concurrent project, of which, the author also shared a role [22]. All analysis of the results from studies conducted with the prototypes was done entirely by the author.

Some work presented in the thesis has been published as papers or presented in consortiums. The details of these contributing papers can be found in Appendix A. Finally, the topic of the thesis was selected to appear in the EPSRC Nation of Pioneers competition which celebrates transformative and pioneering research by UK-based doctoral students ${ }^{4}$. Here, the author reached the final in the category of creative computing for the digital economy.

Note, at the end of each research contributing chapter the author declares their contributions, and what support was given by fellow researchers and $\mathrm{PhD}$ advisors.

\footnotetext{
${ }^{4}$ EPSRC Nation of Pioneers: https://bit. Iy/2MoyVII
} 


\section{Chapter 2}

\section{Related Work}

In this chapter, we discuss the related research within the scope of the thesis as our goal is the design and development of new deformable devices for digital art user experiences. We can see in Figure 2.1 an illustration of the key areas of related work we consider relevant and where the overlaps lie. We identify an unexplored area of deformable interfaces for tangible art interfaces, which is demonstrated by the pink area of the diagram. We also consider that all the work discussed in the thesis has some form of mobile aspect. To incorporate this property we also considered papers that had elements of augmentation on mobiles devices, tangible or deformable. We categorised this as research where mobile devices have had some form of additional physical input or output modality added to them.

We begin by outlining the field of tangible user interfaces in order to show the progression towards deformable technologies, and we discuss augmented mobiles where additional TUI controls have been added to enrich interactions. After defining this work, we move to documenting the key research within the space of interfaces for digital art and design. This includes a discussion of stylus and canvas technologies and other unique forms of physical interactions. Here, we also include how other researchers have conducted their studies with artists and designers to highlight this informed method of user research with such groups.

We then expand our focus by considering areas of deformable interfaces that 


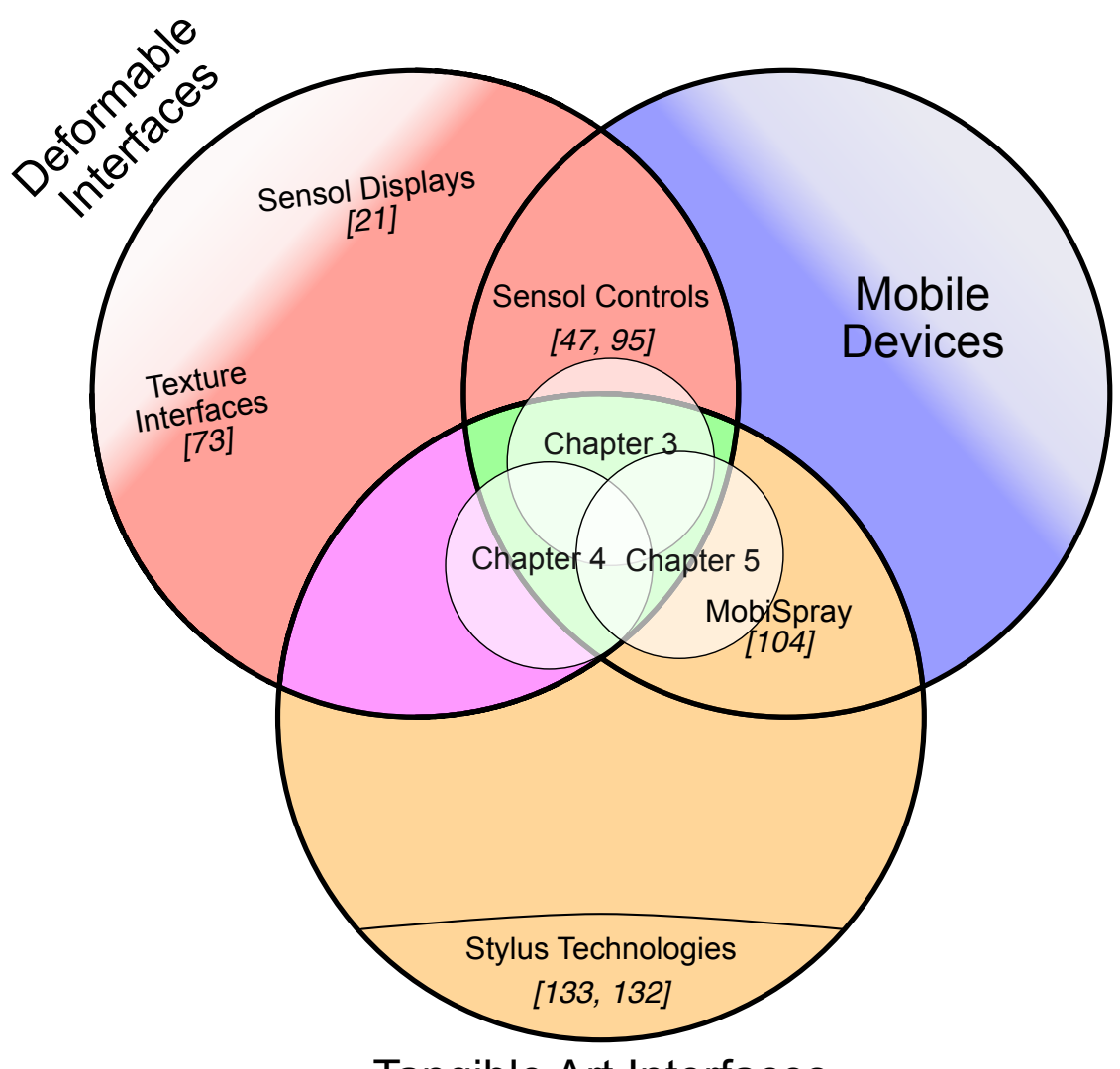

Tangible Art Interfaces

Figure 2.1: This shows key areas of related work we consider relevant to the work presented in the thesis. The white circles highlight the areas in which the prototypes sit. Our focus is on the intersection of art interfaces and deformable interfaces. Some of the work also overlaps with mobile HCI due to the nature of the prototypes

infuse the prototypes demonstrated in the thesis by discussing the different types of physical input and output devices developed for dynamic tangible interfaces. This includes a discussion of related actuated interfaces, dynamic texture interaction and modular devices. We conclude with an overview of the related work in this area, summarising the key issues and areas that we build upon in this thesis. 


\subsection{Tangible User Interfaces}

Before we outline the related work form the tangible art tools and deformable interfaces we acknowledge the key Tangible User Interfaces (TUI) literature that paved the way for this work and the key influences [107]. TUIs where first formally defined by Ishii et. al. in the Tangible Bits paper [45]. The paper outlines how a TUI is a "user interface in which a person interacts with digital information through the physical environment". Key research papers have studied how we interact with tangible interfaces offer a more stimulating user experiences when compared to their graphical counterparts $[18,38,131]$. The GUI version can sometimes provide a better performance [144] and Ishii et. al. continues this discussion in a succeeding paper about the evolution of TUIs [41]. This article first highlights how TUIs are shifting away from discrete objects to continuous tangible material, describing elements similar to those common in deformable interfaces. An extensive review of tangible user interfaces by Shaer et. al. outlines the past, present, and future directions of the field concussions. The review concludes that the future of tangible user interfaces lies in continuous tangible material highlighting actuation. These papers further support the shifting interest from TUIs for deformable interfaces in order to take advantage of continuous tangibility.

\subsubsection{Tangible Augmented Mobile Devices}

There is a body of work that demonstrates adding tangible input controls to mobile devices. (e.g. [10, 40, 60, 143]). This extensive work centres around providing tangible interaction on mobile phones via an attachment. Of these examples, we see phone surface attachable controls $[40,143]$ and side attached controls. These tangibles offer interactions such a squeezing/pressure, knobs/dial, sliders and switches [60]. Another example takes advantage of the camera of a smartphone so that physically objects can be placed over it as a tangible control input [136]. We also see 
work that adds input via bending the device, an attached screen or an external attachment $[77,94,111]$. Some devices are capable of actuation that changes the size of the device to deliver information $[17,31]$.

Other types of actuation come from deformation with shape-changing areas surrounding the device $[23,80,86,87]$. In the Morphies paper, the prototypes developed allowed for bending or deforming areas of the device itself [99]. In Haptic Edge, controls dynamically change along the edge of the device [47]. Work also supports the use of the back of mobile tangibility to support user interactions $[15,16]$. Yet, we see that no work has studied providing back of device actuated textures or looked at providing tangible interaction tailored to the digital art, and furthermore nothing at the interaction of mobile shape-changing for digital art.

\subsection{Tangible Tools for Digital Art and Design}

There is a large body of work that explores enhancing interactions for digital art and design. As we aim to explore how to bring about new deformable tools we focus this first section on what existing tangible tools have been developed and what collaborations the researchers had with the arts and designers to inform this research. We define a tangible user interface (TUI) as a "user interface in which a person interacts with digital information through the physical environment" [45]. Specifically, for us, this digital information relates to an artistic activity. This includes interactions based around digital painting, colour picking, desktop publishing and drawing to produce digital illustrations or graphic design work. 


\subsubsection{Painting Interactions}

Researchers before us have explored the possibilities of enhancing interactions with digital art interfaces. Like much of our own work we see elements borrowed from existing traditional painting methods. CavePainting project presents an interface to create 3D works of art in a virtual reality cave environment [52]. It uses physical props and gestures for an intuitive interface for artists, who may not be familiar with virtual reality. We also see an example of tangible painting in an exhibit for a museum [11]. This provided children with a TUI for interaction with paint pots. A similar tangible emulation is MobiSpary [104]. This is a mobile gesture-based control app that provides a natural pointing mechanism for a virtual spray can. Other works, such as Colourful Touch Palette, considers the possibilities of tactile sensations [34]. It introduces "Texture Canvas", a dual touchscreen system where one is the painter's palette and the other a canvas.

\subsubsection{Stylus Interactions}

Work also explores novel stylus technologies to offer finer, more realistic controls $[133,132,83,115]$. In the work of Otsuki et. al., we see the use of mixed reality to enable painting onto physical objects in the real world. This is afforded by its flexible nib. Another related project is FlexStroke, which is an example of a deformable stylus [66]. Its dynamic stiffness ability affords the feeling of different types of brushes. It provides this variability to use its jamming structure. Meanwhile, Baxter et. al. go as far as to imitate virtual brushes [4] with haptic feedback sensations. A further novel painting interface is EMS Painter [13]. This enables an audience to influence a painter's use of their paint brush through electrical muscle stimulation.

Tools such as I/O brush enable drawing with everyday objects as ink [101]. This works by putting the brush on an object to capture an image of the area, enabling 
the user to paint with this image. This demonstrates how digital tools can enable engagement with our physical surroundings. Similar work demonstrates using everyday physical objects as a paintbrush [58]. A related implementation is Mix Brush [85], which uses an innovative colour mixing interaction consisting of a stylus with RGB LEDs wired through its centre. The LEDs light up for each colour the user selects. The pilot study showed that prototype enhanced the user experience and holds potential in the area of child education

\subsubsection{Graphic Design Interfaces}

We also include work that has developed interfaces for designers due to its crossover with artists using colour picking and mixing. There is a large body of work that explores enhancing interactions with digital colour. Of these works many have invested how artists and designers pick and interact with colours. Jalal et. al. work maps out a design space around the perception of colour and uses these insights to develop novel GUI-based colour tools [46]. Another study has worked directly with illustrators [128]. This research demonstrated that illustrators need seamless switching between digital and physical art. We extend this work of exploration by adding a rich and diverse tangibility to digital colour picking, which offers an alternative to GUI systems. Furthermore, in some elements of this thesis, the work with designers surfaced ways in which tangible tools can improve picking a colour for accessibility. Tigwell et. al. looked at available GUI tools, whilst also working with web developers to understand how to build new tools [126]. We build these design considerations into tangible tools, which is demonstrated in Chapter 5.

We have seen examples of tools that help designers have a more engaging tangible experience when interacting with colour $[101,58]$. Related to this, we also see the work that studies the design process of designers to develop graphical tools $[46,68]$. Our work extends this by also exploring the process of designers to understand where we can specifically aid their creative experience in digital. We 
do this uniquely by involving them in the design and explorations of tangible tools for the digital colour picking. We contribute an understanding of their workflow, the constraints they face and a unique tangible interface that begins to address such constraints.

We have extended this work with painting emulations work by working alongside artists to develop further elements of realism in haptic sensations from gels (see Chapter 4). This contrasts with the VR work [52] of transporting the user to a virtual space by maintaining their physical connection to reality. This is similar to the exhibit with [11] MobiSpray [104], which aims to emulate art interactions that exist in the real world. To this we add the sensation of haptic paints adding a further layer of realism.

From what we see in this section, the predominant research into TUI for art interfaces has studied the advancing stylus technologies. Given this, we have taken an approach of looking at areas outside of the stylus, like paint selections and manipulations. We also see that, to the best of our knowledge, no tangible art interfaces have explored specific elements of deformable interfaces. In our aim of exploring deformable interfaces in this space, we extend the level of realism experienced by artists.

\subsection{Working with Artists and Designers}

This is key in grounding our findings into how artists work and how the new interfaces we develop can aid in supporting them in the future. Jalal et al. explored how artists and designers interact with colour [46], using these insights to develop novel GUI-based colour tools.

Other studies have worked directly with illustrators [128] and have identified them as users of both traditional and computer-assisted tools. They worked with 
professional illustrators to develop augmented paper to enable illustrators to zoom in and out of sections in their drawing. After giving it to the illustrators, they found that it improved productivity on paper while enhancing an illustrator's creativity. In other studies we see how designers discover inspirational materials for their work. Funky-Design-Spaces has specifically looked at how designers make and interact with mood boards [68].

Their work looks into the development of interactive environments. They directly routed the development in observations with designers. We take this approach to our work and study how tangibility can support colour tools. The work highlighted in this section paves the way towards thinking about colour mixing in an innovative way. The research [46] highlights that colour picking goes beyond sliders on a screen, while the work of Lucero et. al. [68] shows how highly thought out the design process is. Finally, we consider how the physicality of transitional colour interaction has influenced bringing tangible elements to digital colour tools. Overall, these works have claimed an enhanced user experience and pioneered novel interaction techniques. This is a trend we continued in the evaluations of our prototypes presented in this thesis. We also use this work to inform the ways we worked with the artist for initial research gathering.

\subsection{Deformable Interfaces}

In order to innovate and bring about new and rich interactions with digital art experiences, we look to work that enhances what is capable when pairing the digital and physical. Deformable interface research is an area that examines how tangible interface can not only be physical but can also dynamically change. This idea was introduced by Poupyrev et al. [92], and the work outlined the concept of dynamic 3D controls with a Lumen display [91] as an example. These papers outline the key concepts and example prototypes that demonstrate what it means for an interface 
to be deformable. After these initial papers, a defining vision of deformable interfaces was outlined by Ishii et. al. in the article Radical Atoms [42], which showed a world beyond the TUI and towards transformable materials [42]. Since this, the way in which interfaces can change shape has been explored in a wide range of ways.

We use the technical innovations from deformable interface research to further innovate and expand what is capable with digital art and design interfaces and realworld deformable use cases. In this section, we aim to explore the many different use cases and techniques researchers have employed to build and test deformable interfaces with the field of HCI. To support this investigation we used key review and design space papers to find and understand the field and what had been done before us $[3,93]$. Note there's an extensive amount of work published in the field of HCI, so for this section we focused on the most important papers with the most significant effect on the field and ones most relevant to the work in the chapters of the thesis.

Note there's an extensive amount of work published in the field of HCI, so for this section we focused on the most important papers with the most significant effect on the field and ones most relevant to the work in the chapters of the thesis.

\subsubsection{Displays and Controls}

Key forms of shape-changing displays can deform their entire surface via a grid of actuators $[21,27,64,76,95,135]$. This form of interfaces combines screen deformations and dynamic tangible controls. An early form of demonstrating these grid-based interfaces is Relief [65]. The researchers of Relief then further developed this method of actuation to present the tabletop interface inForm. It illustrates the possibilities for an array of visualisations and dynamic affordances alongside implications for adding physical presence for telecommunication [21, 64, 43]. The 
supporting papers outline frameworks for dynamic affordances and constraints [21]. We also see how inForm can be deployed for physical telepresence to enhance communication over the internet [64]. InForm type devices are also explored in the context of dynamic workstations [135]. Recently, the display has taken the form of Materiable, where the shape-changing display could imitate dynamic properties, such as flexibility, elasticity and viscosity [76]. This enables richer embodied interaction and perceptions of rendered materials.

An early example of using deformable displays on mobiles can been seen by tapering a two-dimensional screen onto the back of a mobile [31]. This was used to communicate ambient information, such as remaining battery life, by physically tapering out the back screen for the user to feel a deformable area. Further enhancement of deformable mobile displays use actuators similar to inFrom [21]. This type of mobile actuation is demonstrated in the work of Robinson et. al. [95]. The work further diversifies modalities of interactions by employing the ability for each extends the use of sensel to act as dials or sliders. The study compared the deformable prototype to a touchscreen GUI. The deformable was better for eyes-free control of continuous parameters [95]. Furthermore, the human factors involved in users reaching and using these discreetly placed sliders is explored by Rosso et. al. to show how size of the motor space impacts performance [97, 98]. Another further enhancement of the use of rods in grid-based displays is seen in TiltStacks [124]. In this work, the TiltStacks prototype can tilt and stack, which surpasses the rigidness of actuators seen in work before it.

We also see the prototyping tool Shape Clip, a tool for researchers and designers to rapid prototype shape changing displays [27]. ShapeClip uses a shape display shader language to determine the length of actuation [137]. This language makes it easier for a researcher or designer to prototype a 3D display without the need for advanced programming knowledge. This work shows how a screen's pixel can rise from the surface to present 3-D elements of a display. The use of 3-D displaying 
has also been harnessed for data visualisation, allowing users to view bar charts as a physical medium $[118,119]$ This work studies the challenges of how we might display information on deformable screens as these new displays are non-rectangular, unlike current popular smartphones, tables and display monitors [106].

Other examples of 3D displays have employed the use of levitating objects, seen in LeviPath [82] and BitDrones [24]. LeviPath uses an array of acoustic waves to levitate beads and create paths by moving them across the array of wave forms [82]. In BitDrones Nanocopter are employed as displays with examples showed of moving screens and individual pixels carried by the drones. The paths are again programmed based on the defined flight paths, meaning that the displays change shape depending on the position of the drones [24]. Deformable interfaces have even be deployed in large scale scenarios for furniture [26] and architectural spaces [44].

We also see work where shape change provides dynamic input controls. Harrison el. al. demonstrated the use of dynamic physical buttons on a visual display [28]. Here, different buttons bubble up from the screen based on the displayed graphics. Input control also extends to examples of flexible interfaces such as MudPad [49] and Force Form [130] in which users can push into the interface. Other types of dynamic controls take position on the edge of the screens [77, 47].

In Haptic Edge, controls dynamically change via translation of the pins positioned along the edge of the device [47]. The pins rise to enable dynamic physical affordances, shape display, non-dominant hand interactions and in-pocket haptic notifications. MimicTile [77], a stiffness deformable, has the ability to recognise several deformation-based gestures. This provides haptic feedback through its flexibility and variable stiffness. This example is a permanent fixture on the mobile device which constrains its ability to deform into a control based on the task. Other examples of side attachments for mobiles have used deformable elements for interpersonal mobile communication and tactile-visual phone conversations [86, 87] Another unique use of deformable elements is seen in HotFlex. This prototype 
demonstrates how 3D prints aid in modifying shapes after objects are printed, giving digital fabricators an opportunity to shape their prototypes without the need to re-print the object [25].

Many of the prototypes such as Morphees [99] and FoldMe [53] have focused on output, while here the whole device will change shape rather than in isolated areas. We intend to focus tangible interaction with the deformations. However, so far these deformable interfaces have provided users with input. Elements are either surface level or "pushed in", making the fidelity binary. There is also work surrounding mouldable tangible interfaces. Work such as Haptic Chameleon [71] and DO-IT [74] proposes that controls with dynamic shapes change during use. The application was a single purpose tangible widget for video editing.

In our work, we aim to further diversify interaction modalities driven by scenarios to explore the physical representations of tools need. These act both as input and output extending this area of work. In exploring these modalities, we want to combine the mechanical interactions of inputs such as sliders and dials with organic interactions styles. Our research interest lies in deforming the screen based on the task at hand, for example, switching between pot and nib or different viscosities of paints (see Chapters 3 and 4). Furthermore, the work outlined in this section shows examples of shape-changing displays that transform to present dynamic controls or some form of output to the user. So far, however, limited work has combined the elements that user deformations provide input and output. In examples that combine both input and output, we see limited output possibilities [21, 64, 76, 135] with output still being the focus.

In [95] we see a greater focus on providing the input with the implementation of dynamically appearing dials and sliders. However, the sides are not dynamic in the size where they appear or when being controlled. In a graphical display, controls can take many different sizes, as do future visions of deformable interfaces. Other control switching interfaces can be seen in $[54,71]$. Here, the deformation 
change functions and differentiate tasks. We wanted to expand this work by changing shapes during operations in order to communicate the state of selections within the functions.

In the design of deformable displays we see great work that aims to classify and map out possibilities for deformables at a defined field of interface. This work ranges from public ideation $[20,114]$ the characterisation of these actuation techniques [120], understanding and characterising of movement qualities [59, 121] and using design fiction [113]. Finally, we highlight the work of Kirk et. al. that raises the issues in designing hybrid interactive surfaces, exampling how it can be difficult to get the appropriate balance between the physical and digital parts of an interface [56].

A good example of deformable controls that provide a haptic output during tangible interactions is Haptic Wave [122]. This provides haptic actuation during interactions with a linear movement feedback on a slider's thumb. Yet there still remains research into concurrent actuating controls (explored in Chapter 3), concurrent texture interaction and haptic feedback (explored in Chapter 4) and dynamic screen changes based on shape (also explored in Chapter 4)

Finally, in a review of shape-changing interface literature, there was a call for more qualitative research and scenario-based research [93]. In this thesis, we have addressed these open calls by using deformable interface research to inform the building of our prototypes for an art and design scenario were the artists give qualitative opinions of what we developed, and provide realised examples of deformable interfaces applied in a use case of digital art.

\subsubsection{Deformable Haptic Feedback}

The nearest example of deformable controls that provide a haptic output during tangible interactions is Haptic Wave [122]. This provides haptic actuation during 
interactions with a linear movement feedback on a slider's thumb. Yet, there still remains research to explore these types of actuating controls and the possibility of using these in multiple scenarios outside of audio productions. Studies show the benefits of using tactile and audio feedback over visual output [8, 36, 35, 90, 140]. These studies highlight the load put on the visual sense when interacting with systems where visual feedback is the dominate output. Another related study explored the timing stimuli through auditory and tactile cues [14]. So far in these comparison studies, the main technology used for tactile output has been vibrations. Our study in Chapter 3 extends this by comparing visual, audio, vibration and shape-change. We note with interest that we see haptic feedback embedded in wearables: for kinetic clothing [5] for feedback gameplay [1] and in CADLens for navigation [29].

A number of works within this area have studied the forms that output shapechanging interfaces can provide [91, 92, 99]. Others have focused on the concept of presenting dynamic tangible controls [21, 95, 54], where interfaces can switch between different types of physical input controls, such as sliders, dials and buttons. In these types of interfaces, we see the flexibility of locations on the screen, but unlike their GUI counterparts, the individual controls do not change form when presented or during interactions. Shiftio shows how systems can provide reconfigurable tactile elements. This allows for dynamic affordances and mobile interaction to elements of the feedback, which are not isolated to a fixed areas of the device. A GUI interface element can take different forms depending on the task. They can also dynamically change during an interaction with mappings to a value or context, clearly seen by the user. The disadvantages of this are its requirement of users' visual attention and not providing any tangible feedback. Providing this type of dynamic change to shape-changing interfaces employs the advantage of tactile feedback when using the controller. This aids eye-free uses and the communication of system states. 


\subsubsection{Flexible Deformable Interfaces}

The idea of dynamic input control was also explored through the use of pneumatically controlled actuations [55], [134], [142]. Early examples include the Inflatable Mouse. This is a volume-adjustable mouse with air-pressure-sensitivity, which allows for input and haptic feedback [55]. The dynamic change afforded zoom functions and fast scrolling using pressure. There was also further discussion of its uses as an emotional communication tool. You can also manipulate pneumatics for tactile responses based on different levels of force from users [134]. These forms of pneumatics limit tangible control based on the force.

Other pneumatic examples include PneUI, where pneumatics were used in a variety of scenarios for shape-changing interfaces, providing a mechanism for deformable actuation [142]. Similarly, Follmer et. al show a mechanism for jamming materials to hold different shapes on demand, for example, changing from a flat surface to a chair that is able to hold the weight of its user. We also note the use of pneumatics for self-healing soft robots. These are used to offer softer, more organic robots to make shape change look more natural and pleasing [123].

Deformable interfaces have also come in the form of non-ridged deformable displays [105, 53, 127, 81, 138]. Gummi is an example of a bendable computer [105] and this is an early example of Organic User Interfaces (OUIs). These interfaces are related to user manipulations of deformable interfaces. They follow three principles: input equals output; function equals form; and form follows flow [37]. Other non-ridged deformable interfaces include examples of foldable displays [53], elastic displays [127], thin-film touch-displays, and stretchable on-body displays [138].

Within these flexible surfaces, some works have embedded actuators that can bend surfaces $[12,19,23,78,84]$. These added actuators allow output as a computer can dynamically change the form of the surface to provide some type of output. In this case, the interface can provide feedback and a new formation, making the 
physical surface dynamic but not quite a soft texture. One the example of this is the ferrofluids or Hydrogels that provided dynamic texture simulation.

\subsubsection{Dynamic Textures}

There have been many technical approaches to dynamic textures for broader applications. We separate these from the flexible interfaces as they are able to drastically change how they feel via actuation. This idea of dynamic texture changing has been explored through pneumatics [28], [134], [142]. Harrison et al. provided dynamic physical buttons on a visual display, changing the feeling of the screen surfaces in targeted areas [28].

Another approach uses microfluidics, as seen in Tactus [141]. We also see manipulated pneumatics for tactile response related to levels of force input [134]. These forms of pneumatics limit tangible control based on the force. We also see examples of work that use mechanical interfaces to simulate textures $[88,76]$. Parkes et. al. demonstrate Bosu as a design tool for soft mechanics that can record and playback 3D motion. Similarly, the project Materiable imitates dynamic properties, such as flexibility, elasticity and viscosity, again using 3D motion [76]. Materiable enables a richer embodied interaction and perceptions of rendered materials.

We also see the use of Ferrofluids in the prototype MudPad [49, 50], which allows users to push into the interface. Similarly, we see the use of magnets for deformable surfaces in ForceForm [130, 129]. Here, the magnets are used to provide deformable change and haptic feedback. Other metal implementations have harnessed gallium for the dynamic placement of liquid-metal droplets to provide visio-haptic feedback [103]. The space of the deformable surfaces has also seen fabric used; in TableHop, fabric is used as a display alongside transparent electrodes to provide haptic feedback and deformable cues to the user [102]. 
The work of Miruchna et al. introduces temperature-actuated hydrogels [73]. They demonstrate adding this to a mobile resistive touchscreen to support tactile feedback. Another paper demonstrated using hydrogel for wearable technologies [51] and we also see its use in our own work for simulating the feeling of paints on mobile devices [110]. We apply this type of interaction to our prototype in Chapter 4. Our goal was to use hydrogel's soft states to resemble the feeling of paint, and its actuation capabilities to allow the dynamic placement of interface elements. We expand the understanding of hydrogel via a study with artists that demonstrates its use for controlling paint mixing.

\subsubsection{Modular Deformable Interfaces}

Modular construction and modification of interfaces is another theme seen in the deformable interfaces literature. This resembles areas of the TUI research where static objects are re-configurable but not dynamic or actuated. We see Tangible Remote Controllers that could be moved and reconfigured on large displays [48]. We also see that Sifteo Cubes used modular re-configurable cube screens that allowed them to move them into and shape or size with each module being aware of its adjacent cube [69]

In the space of deformables we see examples such as Cubimorph, which continues this modularity in a similar way to Siftios, but enhances their linkages and allows stacking, turntable hinges and embedded touchscreens in a smaller form factor. Furthermore, we see this modularisation obtain elements of actuation and dynamic change so that the system has control over the form of the interface $[75,100]$. Zooids fully embodies this notion through its use of swarm technology to self-build the interfaces for the user [62]. Our work takes a modular approach in Chapter 5 which allows artists to take advantage of modular screens to build their own shapes to explore and share digital colour swatches. 


\subsubsection{Classifying Interactions and Actuation}

There have been many papers that describe and classify interactions and actuations of deformable interfaces. First, we see how Morphees presents a framework for the resolution of actuated mobiles [99]. Resolution change was then further explored by Pedersen et. al., this time it relation to people's feelings and perceptions via a largescale video study where users watch handheld devices change shape, but not where prototypes were built or deployed [89]. Similarly, the feelings and perceptions of users were studied in 'Imagined Physics'. This work reviewed examples of shapechanging interfaces and analysed human responses the changes [70, 79]. Other works have considered the use dance and movement for rich expression [39].

We also see classification from Sturdee et. al., this time based on the application. This work classified eight categories of prototype, including enhanced 2D, bendable, paper and cloth, elastic, and inflatable, actuated, liquid, malleable and hybrid [112]. Furthermore, we see a study that explored affordance, system state, and feedback in shape-changing buttons. This work begins to show the maturity of the field and research considerations beyond technical feasibility, but what it contributes to users mental models of interfaces [125]. Across the chapters of the thesis we explore different methods of interactions and consider their purpose for digital art and design.

\subsubsection{Force-Based Interactions}

It is important to acknowledge the research in Chapter 3, in which we use force as a key interaction modality in the first prototype. The first work capable of display with force vector detection was seen in 1984 from Minsky et. al. [72]. Since this, other work has explored force gestures on displays [32, 63, 117]. Heo and Lee suggest that the use of force control can be more of a more natural means of control 
over traditional touch-screen gestures [32]. A follow-up study also looked at the use of shear force, including multi-touch points [33].

There has also been an extensive study into the characterisation of touchscreen gesture input-force [117]. In this study, the users wore a glove with the force sensor in the fingertip. The limitation this gave was that only the thumb and index finger would be able to perform force based gestures. The key results was how the form factor affected the force and gesture completion time. Gestures were longer on a phone, while force was greater on a tablet, and more force was used during walking. This suggests that adding deformable elements could diversify interactions based on the scenario.

Force-based gestures also plays a key role in interaction with buttons [2]. Alexander et. al.'s characterisation of everyday buttons shows how physically modified controls can make critical actions harder to invoke, highlighting the role resistance plays in users' interaction with buttons [2]. When adding force interaction to tangible interfaces, fabricated prototypes have used tubes and air pulses that allow force interaction to be detected via a measured air wave [30]

To date, no significant work into force-based gestures has looked at the concept of deforming the interface to present different forms of interactions based on the task. Also, work so far has not taken into account the ability of the user to push into the interface, deforming the surface of the screen. Our work identifies how a deformable aspect of force in the screen will affect user experience and control. Providing a shape-changing display that dynamically switches modes of control provided force control as a new input modality for shape-changing interfaces and situated these two elements in a digital painting scenario. 


\subsection{Summary}

This chapter has outlined the critical related work in the area of deformable art interfaces and mobile TUIs. We also show how the current direction of the research in art scenarios predominantly studies enhanced workspace environments and high fidelity stylus technologies. Our interfaces zoom in on more specific elements such as colour selection and nib adjustments. We have seen how to bring elements of realism to the experience much like how researchers have copied the fidelity of brushes on a screen.

We also highlighted how impactful work has directly worked with users to develop some of the enhanced interfaces. In some of the work, user research is conducted up front to gain understanding, while some may only conduct follow-up studies to evaluate the performance of their prototypes. Overall, the art interface work shows us that there have been limited studies that directly engaged with artists and designers. In our study, we use both traditional and digital methods to contribute to and extend this knowledge.

We see an opportunity to grow the number of use cases for shape-changing interfaces. As we have highlighted, there is little to no crossover of deformable interfaces into the digital art and design scenario. The work in this thesis looks at what deformable interfaces can be built when informed by the digital art scenario and offers insight into the future of the field.

The following chapters will present novel work within this knowledge gap. We will show a harnessing of the deformable technologies discussed and how we developed diverse sets of interaction techniques for a series of prototypes using actuated controls, dynamic texture and modular displays.

In the next chapter, we outline our first exploration into the types of actuated controls that can be built to control art applications and how they compare to digital counterparts. 


\section{Chapter 3}

\section{Actuated Art Tools}

Based on the related work shown in the last chapter, we began our research by considering the use of deformable controls for representing tools commonly used by artists. We started this investigation by considering the artist's tools such as paint pots, pens and brushes. This consideration highlighted the physicality these tools provide to artists with regards to switching between tools via grasping, dipping and shaping, not presented in digital tools or even tangible tools. We addressed this missing element by building a prototype that utilised actuated controls, as previous tangible tools are limited to their static form.

Therefore we began our research with the question of how to use actuated interface techniques (like the ones seen in Section 2.4.1) to represent physical tools used outside digital worlds. Our goal here was to build a prototype that demonstrates shape changing capabilities for tools commonly used by the artists, such as pens, brushes and paints. Then once we had built the prototype tests its capabilities ageist commonplace touch screens.

In tackling this goal, we demonstrated a prototype capable of switching between representations of paint pots and nibs of pens or brushes within a single system. The prototype also employs force-based interaction to imitate scooping paint or shaping nibs. We evaluated the prototype with users by comparing its performance to a touchscreen tablet with digital sliders for matching paints colours, nib sizes, and 
then a free-form painting task. The results revealed that the tablet was significantly faster and more accurate when making nib selections, yet despite this finding, it saw an improved user experience in the free-form tasks as users found the prototype fun and enjoyable to use. We discuss the prototype's current limits in the fidelity of controls, and the direction we took after the evaluations to further enhance making art tools with deformable elements. The deformable prototype and its evaluation from this chapter were documented and presented in CHI 2017 as a late-breakingwork paper [109].

Considering these findings, we then conducted a study imitating digital controls by building a deformable interface representing a digital slider. We did this by developing a physical slider with actuated functionalities. This actuation meant the user would feel the change of the system state through the shape-changing cursor upon the slider rail. This slider was comparable to other modalities such as sound and vibration and outperformed graphical modalities. We conclude the chapter by discussing the comparison of the two control methods and how the following chapters will move towards a more realistic representation of art interactions.

\subsection{Introduction}

One of the key advantages of deformable interfaces is their ability to represent different physical controls. This gives way to the use of dynamic affordances and constraints [21] that allows UIs to change to better serve their user. This is often done via a physical actuation to change the physical form of the interface to present new physical elements to interact with.

For example, Emergables mobiles [95] present a system in which dials and sliders are dynamically presented depending on the task at hand. They are also positioned dependent, meaning that sliders can appear anywhere on the surface and can be constrained by a required size. Similar to the dials which can also dynamically 
emerge for the users interaction, this type of dynamic control can be leveraged for other scenarios. In this chapter, we explore ways in which we can use deformable controls to present reparations of tools for art interactions.

As discussed in Chapters 1 and 2 interactions with physical art is a rich tangible experience and deformable interfaces provide a rich tangible experience that aims to address the limitations of TUIs. We see in digital art processes that much of the tangibility has been lost, highlighting an opportunity for deformable interfaces to leverage use case scenarios to advance their capabilities. In this chapter, we seek to see what the deformable controls can be made to imitate arts tools.

In approaching this, we study the form of paint pots and tools nibs. We explore how this can be presented in a deformable interface and how the controls simulate gestures for new interaction possibilities, including the use of force to simulate dipping and squeezing or size actuation to simulate nib changes.

We have already shown how others approach designing deformable interfaces. We see some examples of actions to provide an output or stimuli. Other examples are deformed physically by the user in order to provide input to a system. The most recent systems offer some form of input and output, and they use a dynamic output to change the controls and interact using physical manipulation of the control to provide input. This chapter will focus on expanding this work by exploring the addition of force controls in Emergables, in the concurrent deformation while the control is in use, plus the overall theme of art driven scenarios.

Our first prototype focuses on imitating tools from the art world and presenting this in a system that can switch between each. In this system, we explored the use of force input to support the gesture that the tool represents. We gave the prototype to users in pursuit-type tasks and a digital painting task. In the study we gave them these tasks related to the prototype and a tablet interface with digital sliders. The results indicated that, while users were not faster or as accurate using the prototype in the pursuit-type tasks compared to the tablet, we did, however, see higher 
enjoyment using the prototype.

Our second prototype is a result of the findings and feedback we received from the first study. We found that one of the issues users had with the force interaction came from not understanding the state they had reached. So our second prototype would deform in the user's hand as the interaction changed the state of the system. This then provides a concurrent deforming for the system while the user is interacting. We implemented this into a physical slider and compared it to other output modality, such as sound, vibration and visual. The results showed that it was a preferable mode of output to graphical and comparable to sound and vibration.

Together with our first study, these prototypes begin to show how art scenarios can drive the design of deformable interfaces. From our results, we outline how they will inform future deformable interfaces and the benefits users could gain.

\subsection{Deformable Controls Driven By Art Scenarios}

We envisioned a scenario where mobile screens could take the form of the artist's tools. The screen would change into brushes and then dynamically switch to paint pots (see Figure 3.1). The areas of the screen will submerge to form the paint pots. In these areas users can dip, push and swirl around inside the screen. Then areas of the screen will emerge forming brushes, with the ability to grasp and squeeze to manipulate the sizes. These different elements change and deform as required by the artist. The benefits we aim to deliver include bringing elements of realistic painting to digital experience, and providing a multi-modal input that aids user expression. Throughout this we show how we can build these interactions and provide evaluations of the developed experiences.

In order to imitate pots for colour selection and nibs for brush adjustment we look at what other authors have done in TUI-based examples. For pots we see 


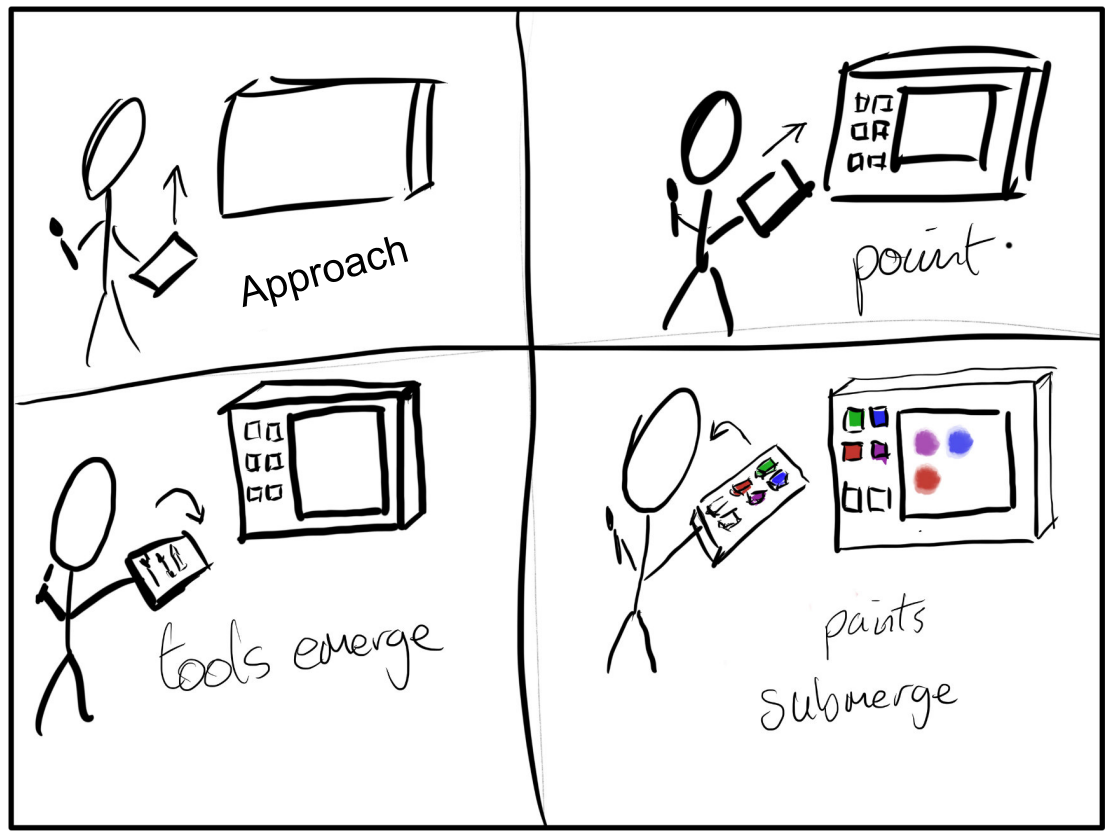

Figure 3.1: Sketch illustrates a user with a deformable mobile and second screen canvas. Here we see the user approach the canvas screen with a deformable devise (Top-Left). The canvas activates (Top-Right). When the user needs to select a brush type and size the sensels emerge to form the brushes (Bottom-left). Then the user needs to select a colour and saturation. So the sensels submerge to form the paint pots (Bottom-Right)

examples of pot-like models used in cave VR [52] and museum installations [11], and for nibs, we see a stylus where users manipulate and change its shape before drawing with it [111]. These systems have shown realistic representations of tools in an individual tangible system, and in this chapter we confront the challenge of combining the two in a single deformable interface.

Our design space extends that of Emergeable mobiles [95]. This space describes how it's possible to manipulate sensels as defined by Rosenberg et. al. [96] to provided a new new level of physical input. In [95] they evolved pixels in touchscreen by creating physical units that can be manipulated in three dimensions and physical size and therefore we also use their definition throughout the thesis for our use of sensel. To obtain the screen deformation described in the scenario the prototype translates into the $\mathrm{Z}$ axis. As well as emerging we also have the sensels submerging. 


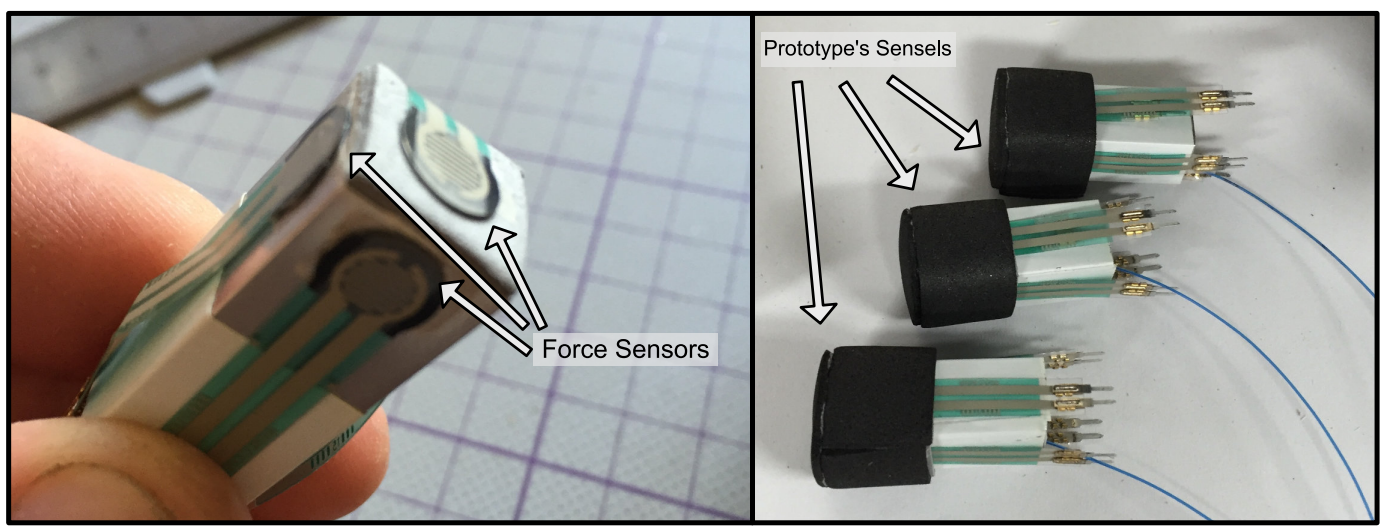

Figure 3.2: These images show the sensels built for the prototypes before they were attached the actuator. We positioned five force sensors on each face of the sensel (Left). Then we encased the sensel in foam (Right).

This allows switching between the brush and paint pot controls.

In [7], Card et. al. outlined a further taxonomy relating to force. We also add this force interaction modality to the sensels. This adds a new modality of interaction to diversify the input control. Encompassing these controls in our prototype brought new sets of interactions. The sensel's ability to contain its own input controls and haptic feedback means that, by acting force on the sensel, the user can make direct deformations to the sensels. The group of force variable sensels allow the user to squeeze and push the interface. This allows them to shape and form the physical material that then translates into the digital. This will give the user feelings of texture and haptic feedback to the actions with the overall aim of complete fluid manipulation of the sensel display. The next section will outline how we built such a system and outlines the design and development process undertaken.

\subsubsection{Prototype Design and Development}

The prototype consists of three actuated sensels with force input modality. Each sensel can actuate to submerge into the 'screen' and emerge from the of the 'screen' 


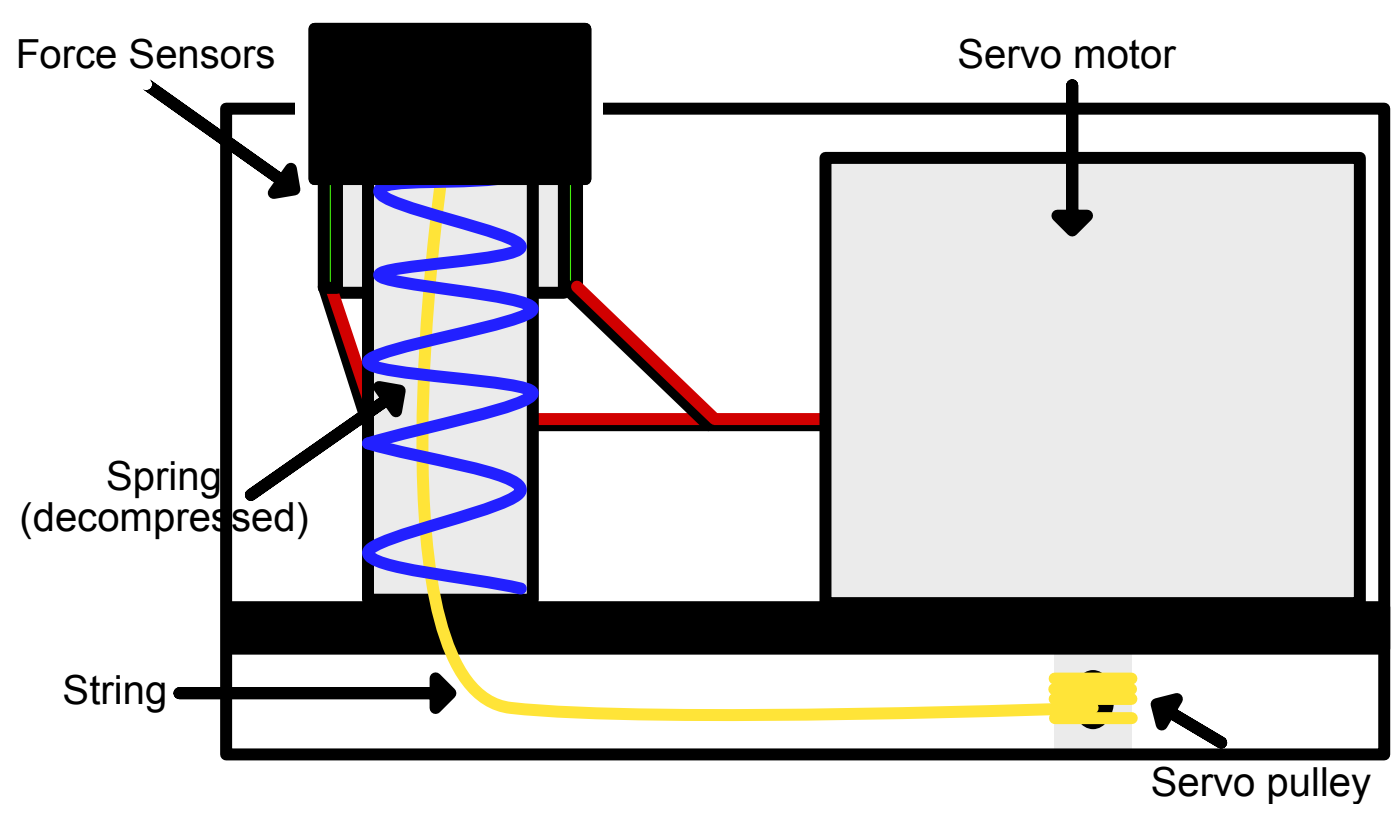

Figure 3.3: These diagram shows a cross section of the string and pulley system used the create the actuation.

(See Figures 3.4 and 3.5). We control the actuation mechanism using a stepper motor with a string and pulley. We attached this string to the top of the sensel and this pulls up and down as the stepper motor rotates. The two paint and nib adjustment controls were co-located as a practicality for the prototype due to the size of the technology need to drive the actuation (A cross section diagram illustrating this can been seen in Figure 3.3).

Inside each sensel, there are two springs. These compress and decompress depending on the rotation of the motor. When the motor rotates, wrapping the string around its pulley, the sensel gets pulled down into the screen and this compresses the springs. In reverse, when the motor rotates to slacken the pulley, the spring will decompress. This will cause the sensel to rise out of the screen.

Each sensel has five force sensors on each square face (as seen in Figure 3.2). The sensors are encased in foam. The foam allows us to take a first step towards fluid manipulation of the sensels outlined in the concept. We used this to surround the force sensors and contain the sensels. This gave the sense of shape manipulation 


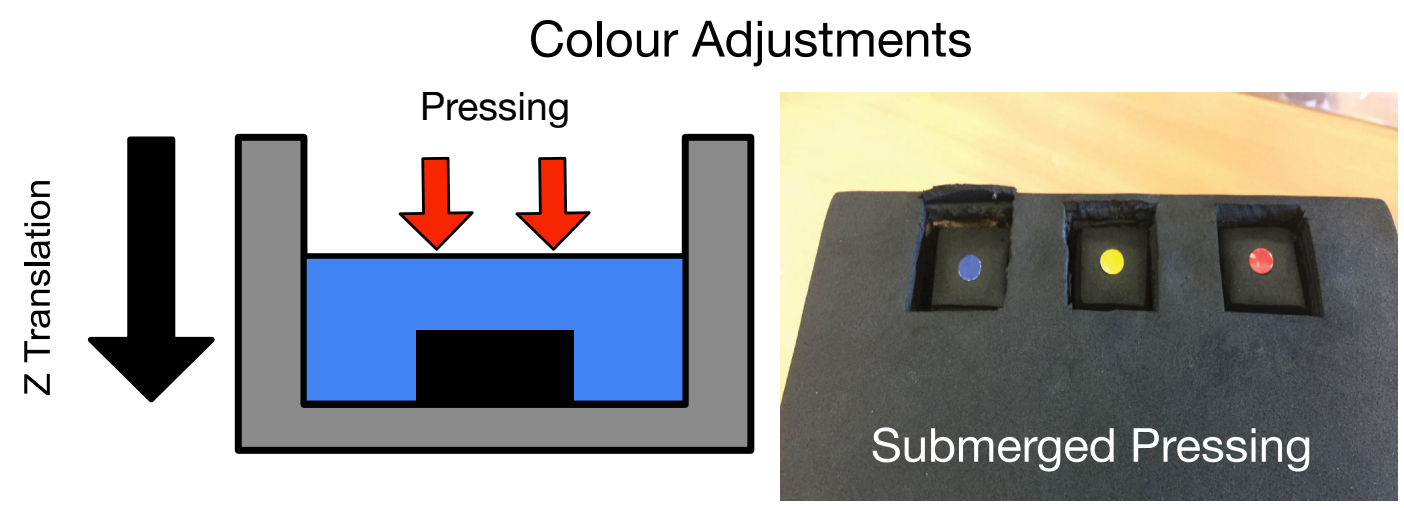

Figure 3.4: This diagram illustrates the colour picking state of the sensel along with force sensing directions. When the sensels submerge it forms a paint pot for paint selection. Here, the force acts down onto the foam and forms the push gesture.

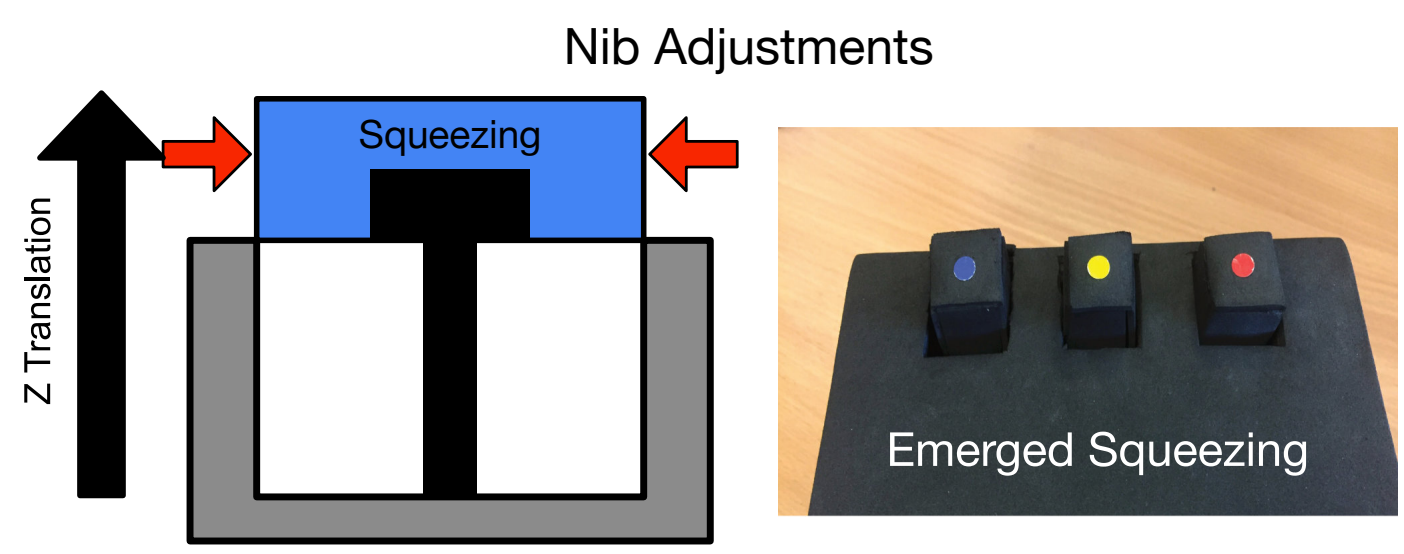

Figure 3.5: This diagram illustrates the nib picking state of the sensel along with force sensing directions. When the sensel emerges it forms the nib for nib resizing. Here the force acts upon the sides of the foam and forms the squeeze gesture.

when squeezed and pushed. The foam provides the user with a textured feedback when acting force upon the sensor. There's a sensor on the top surface to detect push interaction. The sensors around the side surfaces to detect squeezing. The sensels where squared to ensure effective force sensor positioning as they where able to on a flat surface around on each side of the sensel. This gave us optimal force detection as the force sensor gave the most clear reading on flat hard surfaces. A cylinder sensel had the risk of damaging the fragile sensor due to bending and we did not give as constant a reading due to the surface being curved. 
We control the components using an Arduino Mega and RAMPS 1.4 shield. The use of Arduino allowed for rapid prototyping. This meant that none of the component's configurations were permanent until they were working as desired. The RAMPS 1.4 shield gave us the power and programmable control of the three stepper motors. When the deformable connects to the application we perform a calibration. Doing this means that we can actuate the sensels with the correct number of steps for each state. This keeps the system consistent across interactions. Finally, we added a coloured tab to each sensel (red, yellow and blue). This was to minimise cognitive load when picking the control. This aids participants in learning the positions in the user study.

To provide the digital painting scenario, we created a painting application. This accompanies the deformable interface and allows us to log interactions. The application allows users to select colour saturation and nib sizes. They do this by using the force interaction with a sensel-based prototype. The sensels deform by actuating in and out of the screen. When the sensels sink in the screen (see Figure 3.6 they form paint pots of or red, yellow and blue. In this mode, users can push on each to alter the colour saturation. Pushing deeper into the foam makes the colour channel brighter (0 to 255). This maps to the amount of force so that the user can feel that the more force they apply, the brighter the colour.

Once they form the colour desired they push a button selecting the colour ready to draw. When users need to mix colours they simultaneously push down on two of the primary paint pots. This gives a mix of the saturation of each (as seen in Figure 3.6). Once they formed the desired colour users stop pushing on the sensel. If they need a different saturation they begin the push again starting with 0 and rising to 255.

When the sensels emerge from the screen (see Figures 3.4 and 3.5) they then form nibs. In this mode, users can squeeze on each sensel to change the nib size. Each of the three sensels maps to a pen, pencil and brush. Squeezing on the foam 


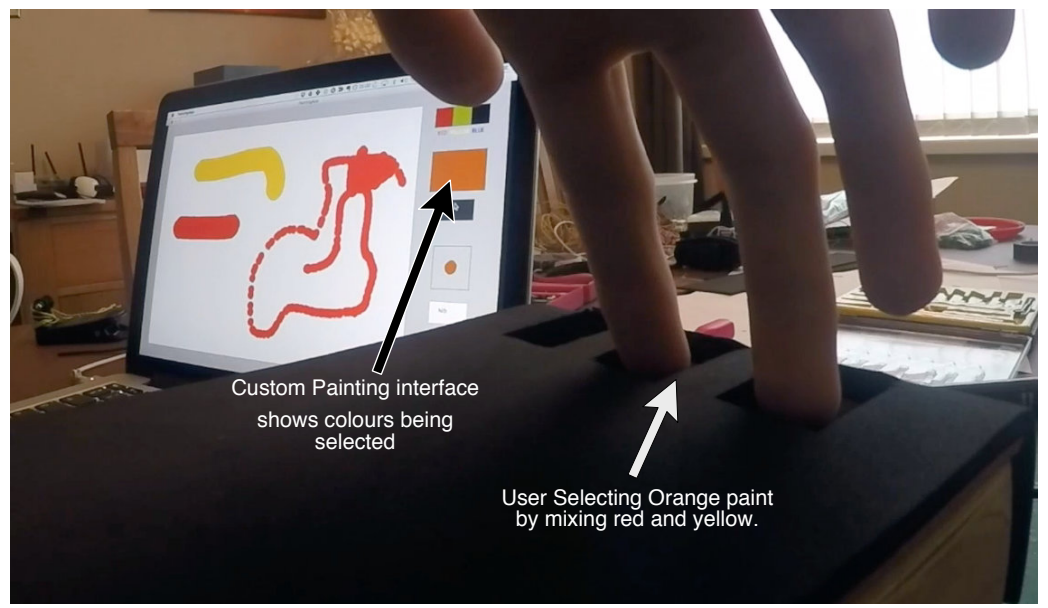

Figure 3.6: This figure shows prototype being used the custom built software.

makes the nib smaller. This is because as you compress the foam it gives the feeling of making the sensel smaller. Once the user forms the desired nib they let go of the sensel. If they need it to re-size they begin the squeeze again starting with a full-size nib.

We also built a tablet interface to use as a comparison in the user study (see Figure 3.7). Using sliders is a common interaction seen in modern day painting applications. We modified the sliders to be comparable to the deformable's controls. We made them square with the same dimensions, meaning that they had the same surface area to grab. They also reset when the users removed their control from the system. This simulated letting go of the sensels. Also like the deformable interface, the system maintains its value until it reaches different selection. We also coloured each slider red, yellow and blue. Like the coloured tabs in the deformable prototype. We limited the length of the sliders as to take up a similar surface area as the deformable prototype. 


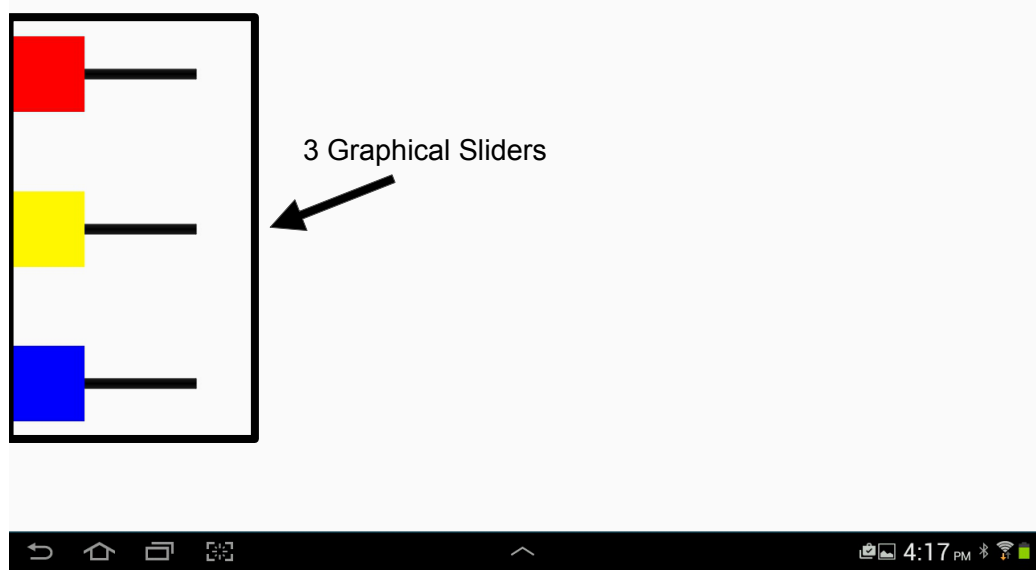

Figure 3.7: This screen shows the interface participants used on the touch screen. Its made up of three sliders positions and sized the same as the sensels on the deformable

\subsubsection{User Study}

In order to test if there was any benefit to using the prototype we design and develop we ran a user study with the aim of comparing the use of actuated tools that our prototype presented with the sliders that are commonly used in their place on touch screens when using art software on a digital tablet environment. Our goal was to measure them both quantitatively and qualitatively via, measuring speed, accuracy and feedback about experiences given by the participants.

\section{Method}

We used a dual interface set-up. The first interface was the controller used as the palette. This was either the deformable or touchscreen tablet. The second was an iPad Pro used as the drawing canvas. This had the painting application mirrored onto it, which allowed the participants to use touch interactions for painting.

We compared our deformable prototype to a touch screen (See Figure 3.7). The procedure consisted of two parts, a matching task and an open-ended painting task. 

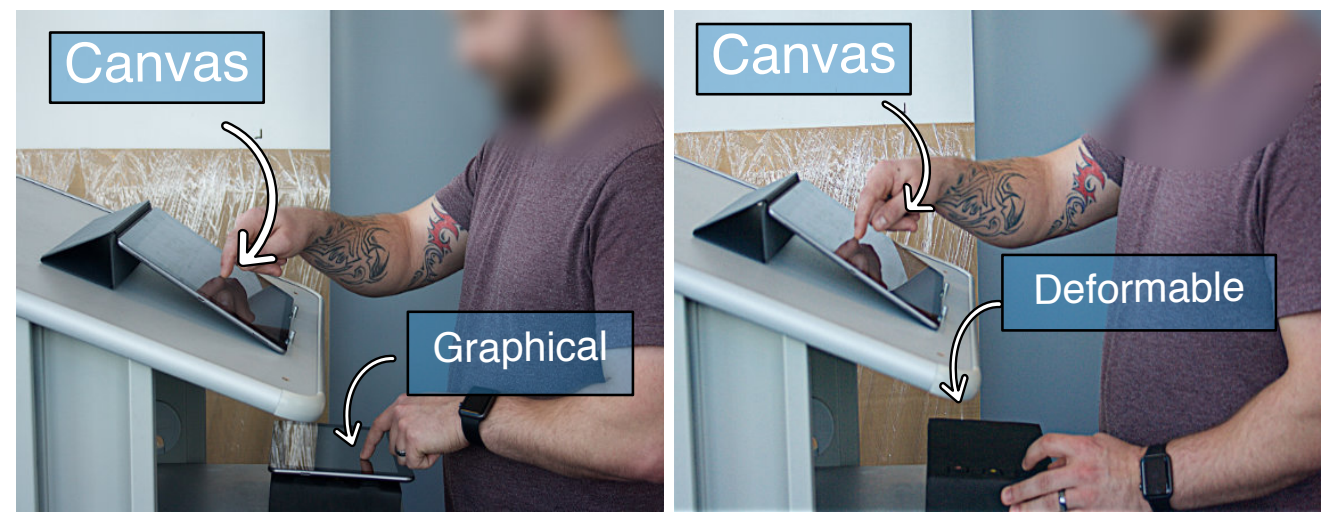

Figure 3.8: Study-up: Participant using the GUI tablet (left) Participant using the deformable(right)

Both parts centre around the digital painting application, each testing the participant's ability to select colours and brush types. For the study, half of the participants used the GUI interface followed by the deformable interface, while the other half used them in the reverse order.

\section{Tasks}

In the first task, we asked participants to use both interfaces to match colours and nibs on screen. The participant makes six sets of matches, each with six individual matches. Each set has a mix of three colours and three nibs. To make the matches, the participant uses either the squeezing for nib or push for paint. Once they perceive that they have made the match they touch 'next' on the iPad. This moves them onto the next match. We mixed the order in which they saw the matches using a Latin Square design. For each task the participants were allowed a practice of one set of colours and one set of nibs to so they understood how to use the interface.

In the second task, we gave the participants a painting activity. We asked the participant to draw four flowers of different colours. None of the tasks we gave the participants had time limits. They were also aware the study was not a test, to remove unnecessary pressure. 


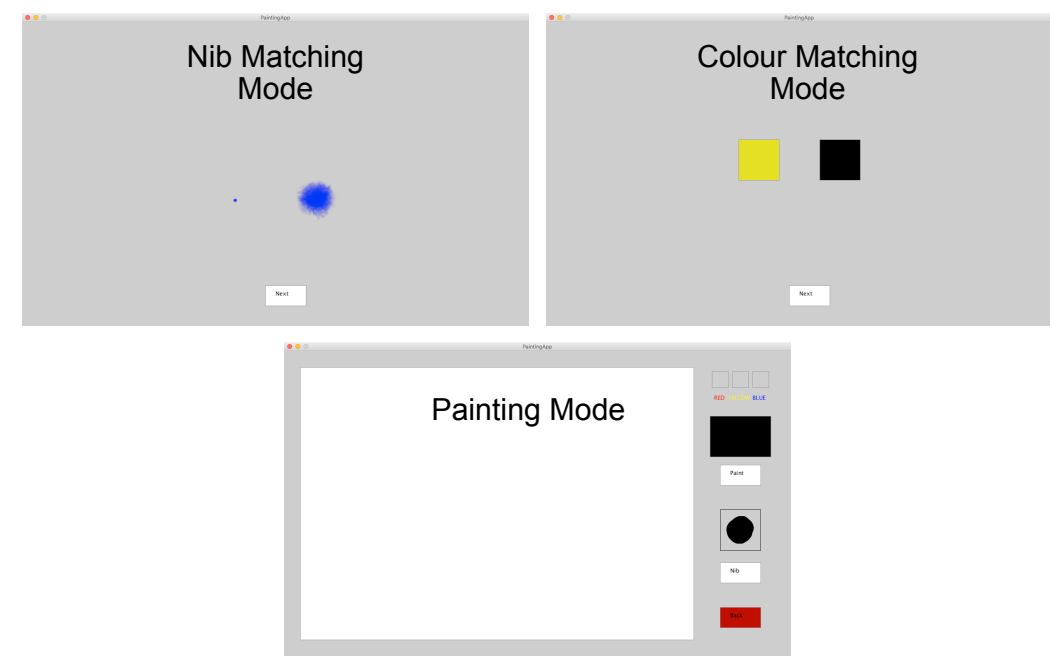

Figure 3.9: Study Modes: Nib Matching (top-left) Paint Matching (top-right) Painting mode (bottom)

\section{Procedure}

We recruited 16 participants $(7 \mathrm{M}, 9 \mathrm{~F}$, aged $21-53)$ to take part in the experiments. We invited each participant to our lab individually for the study. Each of the sessions lasted on average 50 minutes. We compensated them with a $£ 10$ gift voucher.

\section{Measures}

At the beginning of each study, we gave users a demographics questionnaire. This gathered the participants ages and gender along with their hand preference and the number of years using a touchscreen screen. We also asked them to rank how regularly they used digital tablets, digital desktops and physical painting tools.

During the study, we measured the time taken to make the selection and how accurate they were. We video recorded the participants to report their visual attention. During part two of the study, we screen captured all the drawings. After each interaction, we gave the participant a questionnaire to fill in. We concluded with an interview about their experiences during the study. 


\subsubsection{Findings}

\section{Pre-Study Questionnaire}

We recruited 16 participants $(7 \mathrm{M}, 9 \mathrm{~F}$, aged 21-53) to take part in the experiments. All participants had at least four years' experience with touchscreen devices, and one was left-handed. Before the study, we asked the participants how frequently they used physical and digital paintings. The reporting of this is seen in Table 3.1 and visualised in Figure 3.10. The low numbers of experienced painters did not impact the study as we were not testing artist ability, only how it compared as input medium to a tablet.

\section{Matching Accuracy and Times}

We used a t-test to determine if the interface a had significant effect on the ability to match paints $(t(287)=3.561, p<0.001)$. On average users were $4.17 \%$ more accurate when using the tablet interface. We did the same for participants ability to match nib sizes $(t(287)=9.230, p<0.001)$. On average users were $45.67 \%$ more accurate when using the tablet interface. The average error percentages for each task are shown in Table 3.3

\begin{tabular}{llll}
\hline & Desktop & Tablet & Physical \\
\hline Daily & $0 \%$ & $0 \%$ & $0 \%$ \\
Weekly & $31.25 \%$ & $0 \%$ & $6.25 \%$ \\
Monthly & $37.5 \%$ & $6.25 \%$ & $6.25 \%$ \\
Yearly & $6.25 \%$ & $18.75 \%$ & $50 \%$ \\
Not At All & $25 \%$ & $75 \%$ & $37.5 \%$ \\
\hline
\end{tabular}

Table 3.1: Percentage of participants using physical and digital painting, and the frequency they use them on daily, weekly, monthly and yearly bases. 
16

Daily $\square$ Weekly $\square$ Monthly $\square$ Yearly $\square$ Not At All

12

8

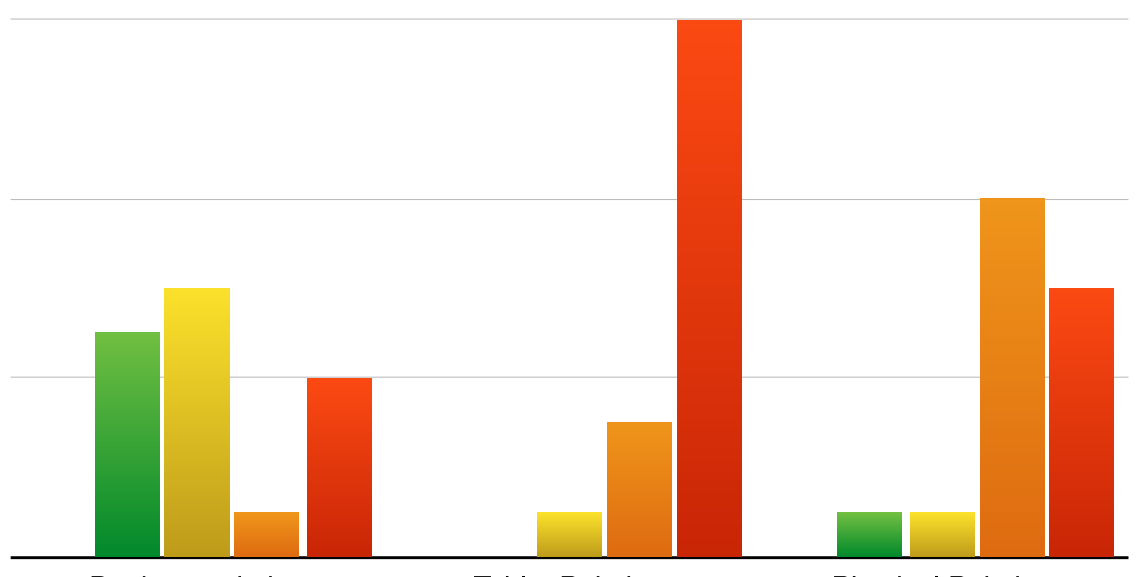

Desktop painting

Tablet Painting

Physical Painting

Figure 3.10: Number of participants using physical and digital painting, and the frequency they use them on daily, weekly, monthly and yearly bases.

A t-test for time showed that the interface had a significant effect on the time taken to match nibs $(t(15)=6.845, p<0.001)$. The selections made using the tablet were quicker. We saw a 3.87 seconds difference between the average times. We also saw a significant effect on the time taken by participants to match paints $(t(15)=3.412, p<0.05)$. There was a time difference of 2.90 seconds with the selections on the tablet quicker on average. A breakdown of the average times are in Table 3.2.

\begin{tabular}{lll}
\hline & Deformable & Tablet \\
\hline Overall & 17.80 & 15.87 \\
Only Nib Tasks & 13.55 & 9.68 \\
Only Paint Tasks & 12.16 & 9.17 \\
\hline
\end{tabular}

Table 3.2: Average Time (Seconds) 


\begin{tabular}{lll}
\hline & Deformable & Tablet \\
\hline Overall & $37.02 \%$ & $11.93 \%$ \\
Only Nib Tasks & $60.39 \%$ & $14.36 \%$ \\
Only Paint Tasks & $13.66 \%$ & $9.58 \%$ \\
\hline
\end{tabular}

Table 3.3: Average Error Ratings (\%)

\section{Experimental Questionnaires}

We analysed our questionnaire Likert-like scale data using a Wilcoxon signed rank test. This allowed us to find significant differences when in the ranking between the two interfaces. The first set of questionnaires were on a 1-7 scale and asked about the matching task (see Table 3.4). Participants' ranking of visual attention gave no significant difference $(z=1.941, p=0.052)$, along with participants' ranking of speed $(\mathrm{z}=1.916, \mathrm{p}=0.055)$ and enjoyment $(z=0.475, p=0.635)$. Accuracy saw a significant difference $(z=2.315, p=0.021, p<0.05)$, with tablets ranking higher with a mean of 4.94 and the deformable's mean of 4.19. Ease of use showed a significant difference $(z=2.671, p=0.008, p<0.05)$. Here, the tablet ranked higher with a mean 5.5 compared to deformable's mean of 3.81 .

In the NASA TLX scores, there was no significant difference in the Temporal Demand ratings $(z=1.307, p=0.191)$. There were significant differences in Mental Demand $(z=2.350, p=0.019, p<0.05)$, with the deformable seeing a higher need for mental demand with a mean of 10.06 compared to the tablet mean

\begin{tabular}{lcccl} 
Question & GUI & Deformable & $\mathbf{Z}$ & $\mathbf{P}$ \\
\hline Visual attention & 5.5 & 5.0 & 1.94 & 0.052 \\
Perceived accuracy & 3.5 & 5.0 & 2.32 & $0.021^{*}$ \\
Ease of use & 6.0 & 5.0 & 2.67 & $0.008^{*}$ \\
Perceived speed & 5.0 & 4.0 & 1.92 & 0.055 \\
Enjoyment & 5.5 & 5.0 & 2.67 & 0.635 \\
\hline
\end{tabular}

Table 3.4: Median Likert ratings (1 low, 7 high) from matching task. Significant results marked with * (Wilcoxon). 


\begin{tabular}{lcccc} 
Question & GUI & Deformable & Z & P \\
\hline Matching Colours & 6.0 & 5.5 & 0.111 & 0.912 \\
Matching Nib & 5.0 & 4.0 & 1.594 & 0.111 \\
Visual Attention & 5.5 & 4.5 & 1.268 & 0.205 \\
Enjoyment & 5.0 & 5.0 & 1.592 & 0.111 \\
\hline
\end{tabular}

Table 3.5: Median Likert ratings (7 high) from the freeform painting task. Significant results marked with * (Wilcoxon).

of 7.19. For the ratings of Physical Demand, we also saw a significant difference $(z=2.832, p=0.005, p<0.05)$. Again, the deformable ranked higher with a mean of 7.06 and a tablet mean of 4.00. For the performance rating $(z=2.460, p=$ $0.014, p<0.05)$ the tablet ranked higher with a mean of 14.81 and a deformable mean 11.00. When we looked at effort $(z=2.033, p=0.042, p<0.05)$ the deformable ranked higher with a mean of 12.06 and tablet mean of 9.88. Finally the frustration rating $(z=2.037, p=0.042, p<0.05)$ also saw a significant difference. The deformable ranked higher with a mean 9.81 and a tablet mean of 5.37.

In the second questionnaire for task two, none of the ranking yielded a significant difference (see Table 3.5). Participants were asked to rate in a 7 point scales the the extent enjoyed using the interface: $(z=0.111, p=0.912)$, where able to mix the colour: $(z=1.594, p=0.111)$, where able to select the nib to match their desired size: $(z=1.268, p=0.205)$ and rate the amount of visual attention given to the interface: $(z=1.592, p=0.111)$.

\section{Post Study Interview}

After the task, we conducted an interview with each participant and asked them several questions. These included the interface preference for each nib and paint selections, notable advantages or disadvantages when using either of the interfaces, whether they would use deformable interfaces in the future, and what other uses they saw. 
When we asked about the advantages of using either interface, five participants reported that the deformable allowed more focus on the task. They stated that " $I$ don't need to see the interface I can just put my finger" and another stated that "I was looking more at the screen for the deformable one". Contrary to this one participant said that "I didn't have to look when had the slider but with the square thing [deformable] I kept looking down to look to see where I was”.

In the context of tasks, seven people preferred to use the tablet for both types of selections. Only two preferred the deformable. The remaining seven mixed across the two interaction styles. Within this group, four preferred the deformable for nib selections stating that "[It] just seemed to work better, seemed easier to understand", while another said that "you could see that instant response between the pressure of your hand and the size of the nib ... I was just thinking, I want that smaller and I was squeezing at the same time whereas in the slider one it just seemed more clunky". The other three preferred the deformable for colour selections and explained their issues with the tablet: "the difference between colour grades wasn't so good or sometimes I [would] miss it [sliders] and I couldn't get it right". Each of this group therefore favoured the tablet for the other selection type. When asked about which interface they enjoyed 11 of them reported the deformable. They explained that they enjoyed the novelty and tangibility of the interactions.

Another theme that emerged was about judging the pressure used. This was an issue for six of the participants. Of these, four explained this to be a reason why they preferred the tablet, as the sliders show the state more clearly. One explained "On the touch screen you can see what you're doing with the sliders you can see the extent you're supposed to go. With the deformable one I just had no idea where to start". These four participants agreed this meant they looked down at the interface more.

When we asked about future uses, two key themes arose. First was using the 
prototype for creative applications. Participants said "I can imagine doing creative work and my sons" or "Anything like drawing, maybe computer aided design” and "I might use it for art stuff as I actually think despite the fact I found it easy on the touchscreen, one my deformable picture was closer to the original one despite the fact it was slightly harder to use ... I think normal artistic stuff is done by hand [and] the fact it's done by hand maybe has a tactile advantage”.

Second, they explained scenarios that need a remote control, for example, "definitely controlling devices when you use other stuff. So you don't have to focus on the tablet like when you drive and change the radio" or "Controls like volume controls, TVs, white goods maybe. Microwaves things like that.”. A final individual suggestion was to use it for people with visual impairments.

\subsubsection{Discussion}

This prototype has introduced actuation for presenting two force-based interactions. We compared this interface to touchscreen sliders for controlling a digital painting application. The results showed that participants were both quicker and more accurate when sliders were used, in the case of making both nib and paint matches. We see that participants found the squeeze gesture difficult compared to the tablet's sliders. Despite the squeeze gesture's high error percentage result, some participants still voiced that they liked using it in the interviews. The participants who favoured it less explained that they struggled with the sensitivity. This could explain the low accuracy.

The difference in average accuracy when making the nib selection was $4.17 \%$. This is still a significant difference, but holds more promise for new gesture types in future development, taking into account the limitations discussed. People in the interviews voiced how it was hard to judge how far they pushed in. Not understanding the state of the interaction may have contributed to the low accuracy. 
For the subjective ratings in the matching task, the tablet ranked significantly higher. This was different to the results from the second painting task where we saw no significant difference in ratings. Combined with looking at the error percentage, we saw participants rated the deformable lower when accuracy was a key element of the task as opposed to the second free-formed task where people had more artistic freedom. We saw in the interviews, that despite people's concerns for accuracy they enjoyed the tangibility of the deformable with three of them even claiming it supported their artistic ability in the second task.

A key discussion point about slider's position was when participants claimed that they were more accurate using the slider. This is because when making selections on the slider you can see that state. They explained that when using the deformable, they found it hard to judge the amount of force needed. This highlights the limitations of foam because it's hard to control the decompression that reverses the value. This supports an investigation into materials that will hold its form and gives users a stronger understanding of their selection's state.

The visual attention ratings has no significant difference in both tasks. This finding contradicts previous works which show that the deformable is better for eyes-free use [95]. Possible reasons for this could be that the task did not need continuous control. The temporal demand rating was not significant so participants did not feel rushed using either interface. This gave them time to look down despite not needing to. Another factor could be the noise of the deformable's actuation drawing their attention. In the interview, participants described their interaction with the deformable as eyes-free despite the results from the ratings.

Participants suggested some future uses for a deformable in the interview. One of these was to use it in remote controlled scenarios. This theme is interesting as remote control objects require eye-free attention control. This is a benefit offered by tangibility [95]. The main common suggestion was creative applications. This makes sense given the scenario of the study and supports the belief that people 
can picture these type of devices in painting style applications despite their current limited form factor.

\subsection{Limitations}

The study has several limitations that could have affected results that directly compare the two interfaces. The deformable interaction styles were very different to touchscreen interactions. This made it difficult to define what interaction should be used on the touchscreen to report a comparison of existing systems.

We chose sliders as these are used in a modern version of painting applications, but the interaction did not directly map to our force controls. This meant we altered aspects of the touchscreen sliders to make a fairer comparison to the deformable prototype. This act may have brought several limitations to the sliders making them different from the applications we sought to imitate. We altered the size of the slider's grab point to be the same size as the deformable's sensel. Doing this made the slider larger than sliders seen in tablet applications.

Another limitation was the user only being able to increment the force and not the decrement. This was due to the issue of when a user let go of the sensel the force disappears, losing their selection. Originally the user would alter the force in both directions and then press a button to 'lock-in' their selection. This was very unrealistic compared to modern applications.

We then detected the user letting go of the sensel, which then locked in their selection. With this simpler interaction came a new limitation of not being able to loosen off, and a result, we altered the sliders so it only incremented in value and reset from the beginning when making a new selection like this interaction in the deformable. This may have hindered the participants' perception of using the 
sliders as people would have been used to dragging them both ways and therefore disadvantaging the tablet.

A key limitation of the study was that the participants were used to using touchscreens and all had at least four years of experience of this. When it came to them using the deformable, each had a short practice run of using the system but lacked the same experience and familiarity. It is possible that this biased the tablet.

\subsubsection{Next Steps}

Based on these limitations and findings, we outline some next steps based on the feedback we got from the interviews. We saw that people preferred the sliders because they could see the progress they had made. This negated the eye-free advantage we wanted to offer. We had hoped the foam would provide a textured feeling on compression, but based on feedback, this gave no sense of position when changing the value.

In future, we intend to explore how we can address this issue. We hope to develop something that dynamically changes to present and hold its states, based on the movement when interacting with the device. The painting scenario can then extend so that once the user squeezes the nib to a certain shape, the sensel can then hold this shape. This means that when they return, they can feel and adjust the same shape. If it then needs to reset, the computer can then actuate it back to its original form.

A further benefit of this computer actuation would be variable friction. This would enable it to be harder/easier to push/squeeze. This dynamic alteration could help users judge and then position it in the system. For example, if you are nearing full saturation of a paint colour, the sensel will be harder to push. This now opens research questions about how we present state in deformable controls. We think this can be broken down into position, size and movement. Positions correspond 
to where the sensels are in relative space on the deformable. In our prototype, positions were actuated and changed by the paint applications. Once this actuation took place, positions stayed the same throughout the interaction. Exploring the midgestured position could provide a better understanding of the state of the selected variables.

Another element is size, meaning the size of the sensel. During the interactions with the prototype's sensel size, alteration was limited to the compression of the foam and could only be altered by the user. Size could be a key factor in maintaining the user's perceptions of the state. Future work should seek to achieve both the computer and user control over the size of sensels. The other factor is the amount of movement the sensel can perform. Again this was limited by the compression of the foam. The amount of movement should change as a dynamic variable based on the task. This means that the user will be able to move the sensel by different amounts depending on what they need to accomplish. For example, larger amounts of movement to reach high numbers or lower for smaller numbers.

Understanding and delivering these elements are a possible next direction for enhancing user control deformable interfaces. It should give the user a better understanding of what different controls can accomplish and a continuous understanding of how they change during an interaction. This study shows that the use of force input and soft materials such as foam made it difficult for users to detect the state of their interactions by touch. This issue impacts the user's accuracy due to mismatching interactions to what is displayed. It also causes an impact on the speed of selection as users would keep needing to reset their colour or nib, which would add time to their workflow. Therefore, despite the users enjoying the tangibility of the system, the force controls overall currently pose inefficiencies. We address these drawbacks in the next section by researching a different form of actuated control as a way to improve feedback from sliders commonly seen in art software. 


\subsection{Actuated Controls for Concurrent Shape-Change}

In comparison to the painting deformable we wanted to address the confusion users had over understanding the systems state, a limitation of the force interaction used. Having a physical slider would bring back what the GUI slider benefited from in the last study with regards to the ability of users to track the position as they drag the slider, helping them understand the state. However, we further aid this by introducing shape-changing for the slider shape to provide physical output to the user. Unlike the sensels in our pervious prototype this slider would will maintain shape and not decompress like the foam.

In this next section, we explore a way to concurrently use deforming tools to communicate output in a tangible way, while the users interact with the control as a possible way to improve on the limitations in the last study. This means that the user can detect the changing state from the physical deformation of the tool in hand, opposed to the depth into a soft material such as the foam paired with a force sensor, as seen in the previous study. This prompted us to explore the use of actuation of controls during use for shape-changing feedback.

To date, research on user interactions with deformable interfaces have seen limited exploration into deforming controls during use. This section explores the concept of concurrent interaction and control deformation. This concept offers the benefits of communicating output and delivering tangible context to selections. For example, using the slider on a physical drawing tablet for use with Photoshop. In this scenario, an artist selecting their nib size would grab the slider thumb, and as they slid it along its track, the nib size would adjust. Only, in this deformable version, the size of the nib would directly map to the size of the slider, and therefore the artist would physically feel the size of their chosen nib in their hand. In order to achieve this feeling, in this chapter we demonstrate a possible prototype to test the viability of achieving this scenario. 
We explored how we can use this concept by designing a deformable slider that shape-changes during use. Our implementation involved prototyping a slider where the curser alters its size based on its positions and output. We evaluated this prototype in a user study where participants were asked to differentiate and select the largest and smallest values. We decided on this task because if the slider is intended for nib sections, the user's ability to recognise the sizes is an important feature. We first conducted a pilot study to test the viability of the prototype. Following this, we conducted a full study where participants used the prototype with the shapechanging mode along with the comparison feedback modalities sound, vibration and light.

In the studies, we measured speed, In the studies, we measured speed, accuracy, and ability for eyes-free use. Our findings show that the shape-changing modality on a slider could be a viable option to deliver additional contextual information. We see that it outperforms current dual screen graphical outputs for accuracy and subjective ratings. It also holds possible advantages over the currently deployable methods of sound and vibration.

\subsubsection{Shape-Changing Slider Cursor}

Exploration of the possibilities shape-changing interfaces pose to the future of $\mathrm{Hu}-$ man Computer Interactions is a growing area of research. Such interfaces are combining the flexibility dynamic change of graphical interfaces with the added befits of physical tangibility. A number of works within this area have studied the forms of output shape-changing interfaces can provide (e.g. [91, 92, 99]) Others have focused on the concept of presenting dynamic tangible controls (e.g. [21, 54, 95]).

In examples where interfaces that switch between different types of physical input controls, such as sliders, dials and buttons [95], we see the flexibility of locations on the screen but unlike their GUI counterparts, the individual controls do 
not change form when presented or during interactions, such as dragging a slider or twisting a dial. A GUI interface element can take different forms based on the task, and can dynamically change during an interaction with mappings to a value or context, which is clearly seen by the user. For example, in software like Photoshop, the size of a nib would update in real time as a slider GUI control is used to adjust it. However, a disadvantages to this is the requirement of the user's visual attention and lack of tangible feedback. Providing concurrent shape-changing with interaction gains the advantage of the tactile feedback when using the control and aids eye-free use via communication of the system state.

In this section, we introduce the concept of concurrent interaction and control deformation. This is where the controls formed will change during the user's interactions. We use control deformation to explore how size can inform output and context while users select system variables. We implement this concept by prototyping a slider control where the cursor can dynamically change its form based on mapping to the task and selection. We explore the viability of this slider through an abstract task that tested the user's ability to detect the sizes from the sliders changing shape, much like if the slider were used for selecting a nib size in art and design software.

\subsubsection{Prototype Design and Development}

In order to test how the concurrent tangible and deformable interactions support user experiences we designed and developed a tangible slider prototype where the graspable cursor can change its size depending on its position on a track (see Figure 3.11). The track was made up of two bars on which the cursor could sit and slide from left to right. On one side of the track, we attached a distance sensor. This tracked the position of the cursor in order for it to be communicated to the application in use. This application then maps the position value to generate an output. This communication is done using an Arduino and serial connection. 


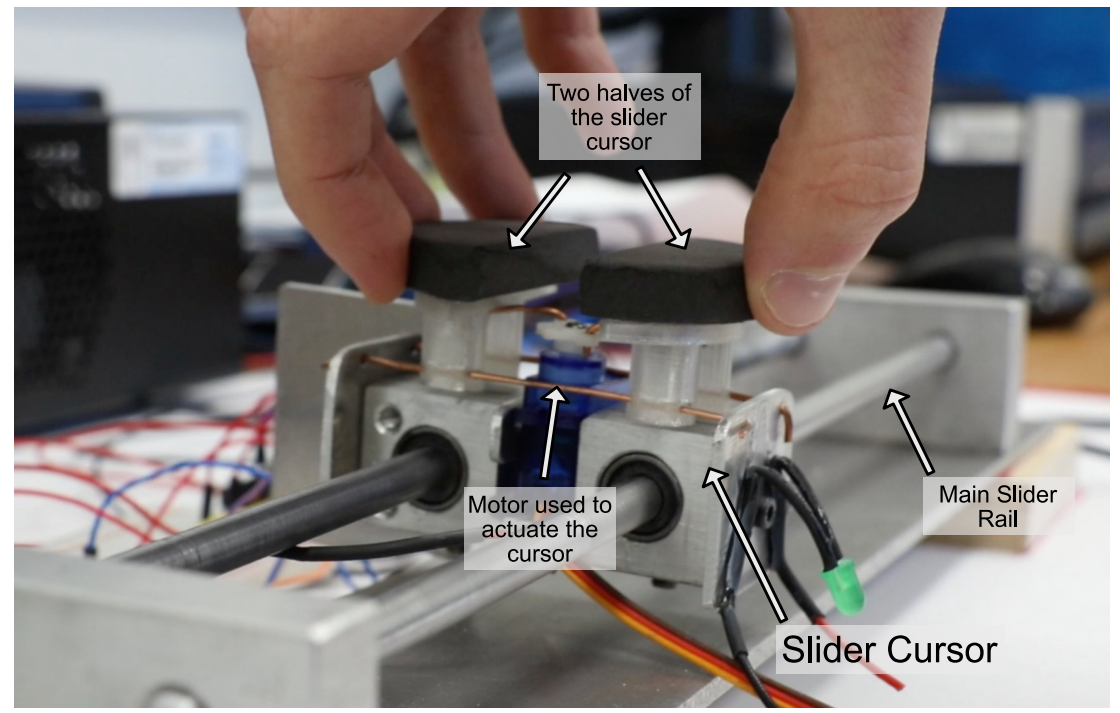

Figure 3.11: Deformable slider implementation.

The cursor is made up of a motor encased in a metal carriage. The main carriage contains two bearings that sit on the track's metal bars. These allow the slider to move along the track. Attached to either side of the motor is the cursor's grips. The grips are made up of 3D printed semi-circles, that when placed together make up the cursors starting state. They are attached to the motor via a metal wire. This wire will expand and contract based on the angle of the motor. Hence, it alters the size of the cursor by pushing or contracting the two sides.

This motor is also controlled by the Arduino. The application can send values that map to adjust the angle to generate the desired output. This creates a feedback loop. The user moves the cursor to the desired position; the position is communicated to the application; the application updates output based on the position; the application then sends the cursor size value based on the position of the motor; the motor then adjusts the size of the cursor mapped to the value, and at this point, users will feel the changed value. The application paired with the slider displays boxes on a screen. Each has an associated value outputted via the cursor size. The boxes can be selected by moving the cursor along the track. As a new one is selected, the cursor will change, as described. The larger the value of the box, the larger the 


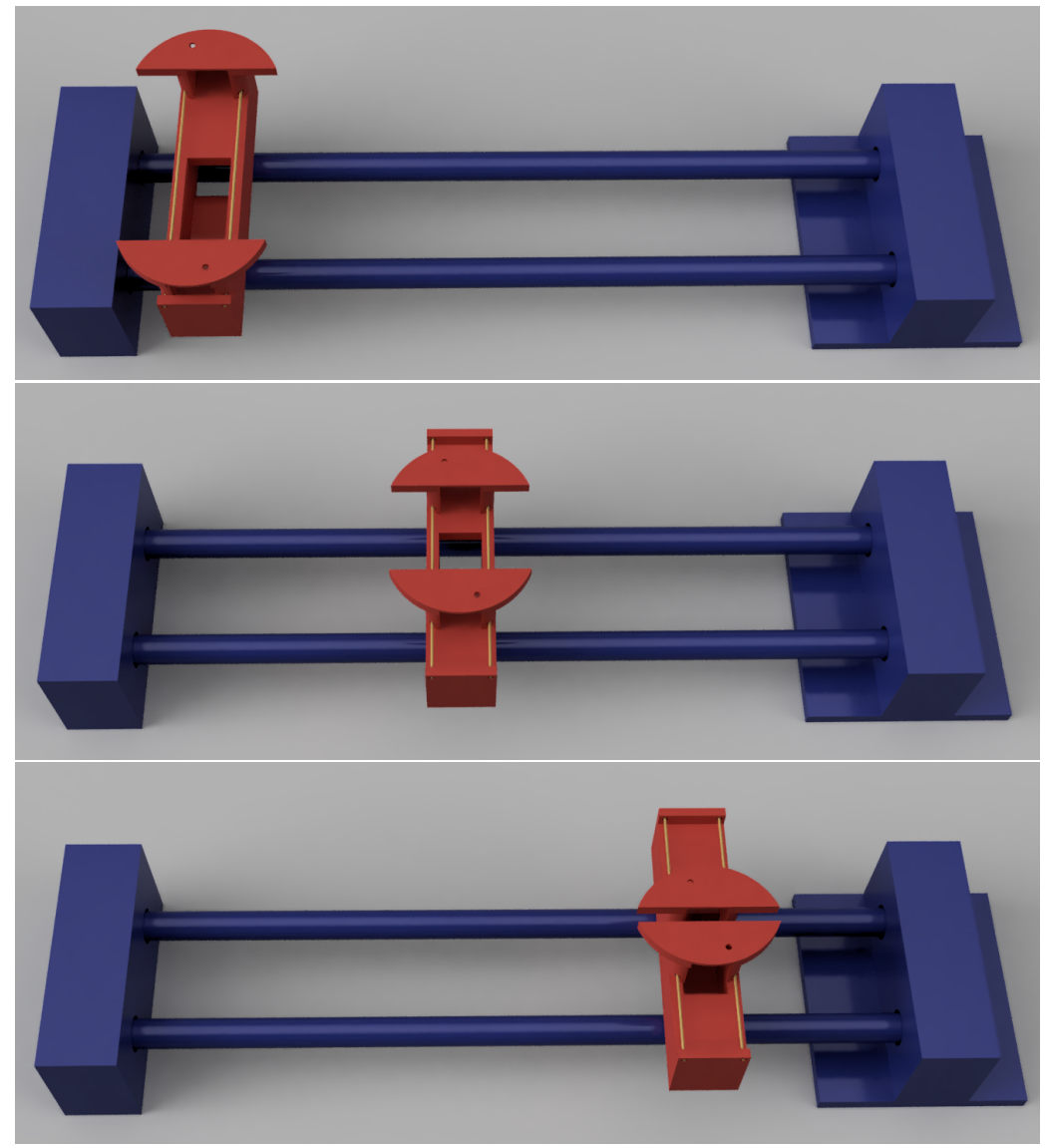

Figure 3.12: Diagrams illustrating three possible stages of deformation: Fully open indicates the largest value; in our software this was 10 (Top), Mid stage open; in our software this was 5 (centre), fully closed, indicating the smallest value; in our software this was 0 (Bottom)

cursor will expand (See Figure 3.12).

\subsubsection{Initial pilot study}

To gather early feedback on our deformable slider prototype, we conducted a labbased pilot study with six participants (5M,1F, aged 21-33, 2 left-handed). Our aims were to determine the robustness of the prototype, whether the user can differentiate size changes in the cursor, and whether the user can determine numeric 
information from the cursor size. We also gathered subjective comments for future improvements and use cases. Studies took, on average, 30 minutes each. We compensated participants with a $£ 5$ gift voucher.

\section{Set-Up}

The set-up of the study involved participants using our slider interface to control a custom application. This application was displayed on a screen directly in front of the interface. Participants sat at a desk to use the set-up and used whichever hand they found most comfortable in order to interact with the systems.

\section{Tasks}

The tasks in each study were split into two parts. The first is a set of closed tasks which tested their ability to determine the points on the slider where the cursor was smallest and largest. This was followed by a graph marking task where participants marked points on a graph based on the cursor size. After each task, they were asked to fill out a Likert-like questionnaire giving scores out of 7 for: visual attention, ease of use and enjoyment. Participants were asked to complete the tasks as quickly and as accurately as possible, but we did not impose a time limit.

In the first task on the screen, there were selection boxes labelled with letters. Using the slider, the participant could move between the boxes. Each box had its own size that translates to the slider's curser. Each box size was on a scale of 0-10 with no boxes in a single set being identical. The participant determined which box was largest and smallest and indicated this to the researcher. Once determined they pressed 'ctrl' moving them to the next set of boxes. Users completed this for two boxes, then this was gradually incremented to six boxes. This made five pairs of small and large selections for each number of boxes. 


\begin{tabular}{ccc}
\hline Number of boxes & Biggest Box selections (\%) & Smallest Box selections (\%) \\
\hline $\mathbf{2}$ & 3.70 & 5.56 \\
$\mathbf{3}$ & 7.41 & 6.67 \\
$\mathbf{4}$ & 21.21 & 3.85 \\
$\mathbf{5}$ & 13.16 & 6.09 \\
$\mathbf{6}$ & 12.28 & 12.50 \\
Overall & 11.552 & 6.934 \\
\hline
\end{tabular}

Table 3.6: Error Rating of Selections (\%)

In the next task, participants used the slider to traverse points of on a graph. They were asked to draw and plot the graph on the provided graph paper based on the slider's cursor size changes when traversing. On screen, they saw which point the slider was at but not the graph itself. All plots were between the values of 0 10. Each participant completed three graphs in total, each with a lower standard deviation of value than its predecessor.

\section{Procedure}

Each study began with participants filling out a demographics questionnaire. They were asked to perform the size determining tasks with the prototype followed by a short Likert-like questionnaire. Then, they were asked to use each interface to perform the graph marking task, and this was followed by a short Likert-like questionnaire. We concluded with an interview about their experiences.

\subsubsection{Findings}

In Table 3.7 we see the average time and the number of switches and error ratings of the size determining task. Each table column corresponds to the number of boxes in the selection. The final row shows the overall number. Whether the user was selecting the biggest value or the smallest held no significant difference $(\mathrm{t}(29)=1.731$ $\mathrm{P}=0.094)$ in the percentage error ratings. 


\begin{tabular}{ccc}
\hline Number of boxes & Average time (ss) & Average No. of switches \\
\hline $\mathbf{2}$ & 12.10 & 3.72 \\
$\mathbf{3}$ & 15.26 & 11.08 \\
$\mathbf{4}$ & 23.94 & 17.48 \\
$\mathbf{5}$ & 25.83 & 30.40 \\
$\mathbf{6}$ & 31.79 & 40.80 \\
Overall & 21.78 & 20.69 \\
\hline
\end{tabular}

Table 3.7: Table shows the number of switches and time taken for the participants to select the boxes.

For the graph selections, the average time and the number of switches and error ratings are seen in the table below. We see the error rates increase as the standard deviation lowers but there was no significant correlation here with this size sample. In the graphs, the users correctly plotted $89 \%$ of the gradients between points. So even if the value was not correct many were able to determine if the next value was higher or lower.

The results of the questionnaire data collected from the size determining task are seen in Table 3.9. The table shows the mean ranks of visual attention, the ease of use and enjoyment. The rankings were marked between 1-7.

We transcribed all the interviews and analysed the scripts using a thematic analysis. The first theme for us was the issues that surfaced about the prototype. Some users reported that the slider skipped values or jumped between two values if it was not directly on either. This was reported to be more frequent as the number of values on the slider increased. Of the advantages the interfaces provides, we saw that

\begin{tabular}{ccccc}
\hline Graph & Standard Deviation & Time (ss) & No. of switches & error (\%) \\
\hline $\mathbf{1}$ & 10.37 & 76.08 & 84.80 & 14.00 \\
$\mathbf{2}$ & 9.72 & 67.78 & 60 & 15.33 \\
$\mathbf{3}$ & 7.59 & 41.32 & 39.20 & 34.13 \\
Overall & 9.23 & 61.72 & 61.33 & 21.15 \\
\hline
\end{tabular}

Table 3.8: This table shows the error ratings, the number of switches and the time taken for the graphs to be drawn by the participants. 


\begin{tabular}{cccc}
\hline Questions & Visual Attention & Enjoyment & Ease of Use \\
\hline Task 1 & 3.83 & 5.5 & 5 \\
Task 2 & 4.83 & 5.83 & 5 \\
\hline
\end{tabular}

Table 3.9: This table shows the mean rating from the questionnaire given to the users.

people described not needing to look down at the interface when interacting. They described enjoying the novelty of the interaction and mainly discussed how they enjoyed the haptic feedback and the tangibility of the device.

When we discussed the size changes, the users spoke about how in the first task the extremities were easy to find. It only became harder when they needed to choose between similar values that had large gaps of other values between them. A participant described "where box A would be close to box E in a six-box scenario, it would be a lot harder to differentiate which was smaller". Despite this, most found it easy to select two similar values when they were closer together or adjacent. One participant stated that "if you know what was small and what was big you could easily differentiate them without any context." All the participants reported that it got harder with more boxes. One participant informed the following "I think to have scale zero to 10 was good, but anything bigger than that and you would be confused" This gives us consideration for the next version.

During the interviews, the participants suggested many improvements. Five of the participants suggested making the deforming cursor bigger so that it would fit into a palm grip. One of the participants suggested providing more haptic feedback for the slider position. For example, it would click with each moment. Another participant suggested how it should transform in complete 3D space. Participants also suggested uses for the device. Four of the participants thought it would be useful for people with visual impairments. They spoke of other tasks for the controller such as volume controls and zooming. One described using it for discreet notifications on a wearable device. Other suggestions included video games and 3D modelling to 
provide a sense of scale and translating virtual 3D to physical dimensions in order to feel the size.

\subsubsection{Discussion}

We see that the shape-changing slider is a viable output mechanism with low error rates and some positives responses from the user. The study also highlighted the technical issues to fix related to calibration improvements. It also highlighted the visual influence. In order that the full study can truly evaluate the eye-free element, we would need to cover the slider and muffle the sound.

\subsubsection{Comparison to different output modalities}

In our controlled pilot study we learnt that our prototype was robust for use and that users were able to use it and understand its output. We also discovered a number of improvements that could be made to make it a fairer test so that we can evaluate it against different outputs in the next study. This includes covering the interface and making the number of boxes on screen appear in a random order more akin to uncontrollable real-world scenarios.

In this section, we explore how we took the shape-changing slider and compared it with other output modalities, to further evaluate its viability. The slider now has four different modes: shape changing, audio, vibration and light. Each different output modality would provide the information. Shape changing provides the information via the slider deforming by expanding and contacting. Audio feedback provides the information by playing an MDI tone where the pitch is altered to be higher or lower based on the selected value. Output via vibration is provided by the intensity, and the more intense the vibration is the larger the value. The vibration module was added to the slider carriage. Finally, light feedback was provided via 
a 1.8" TFT screen set to a white background. Here the output was dependent on brightness, and the brighter the screen the higher the value.

There have been many studies that show the benefits of using tactile and audio feedback over visual output $[90,8,140,36,35]$. These studies highlight the load put on the visual sense when interacting with systems where visual feedback is the dominant output. So far in these comparison studies, the main technology used for tactile output has been vibrations. Our study extends this by comparing visual, audio, vibration and shape change.

\section{User Study}

The lab-based user study was conducted with 16 participants, of which nine were female and seven were male, and all were within the age range of 20-66. One participant was left-handed and nine had some form of music qualification. Studies took on average 60 minutes each and participants were compensated with a $£ 10$ gift voucher. The aim was to determine when engaged in tasks that require constant visual perception such as scenario explore with the artist and canvas, what output modalities can best provide secondary quantitative information via the cursor of tangible slider controls. We compare our four output modalities, which are shape changing, audio, vibration and light, as the output modalities.

The slider has four different modes: shape changing, audio, vibration and light. Each has a different output modality for providing the information. Shape changing provides the information via the slider cursor and deforms by expanding and contracting. Audio feedback provides the information by playing an MDI tone where the pitch is altered to be higher or lower based on the selected value. Output via vibration is provided by intensity, and the more intense the vibration is, the larger the value. Finally, light feedback was provided via a 1.8" TFT screen set to a 


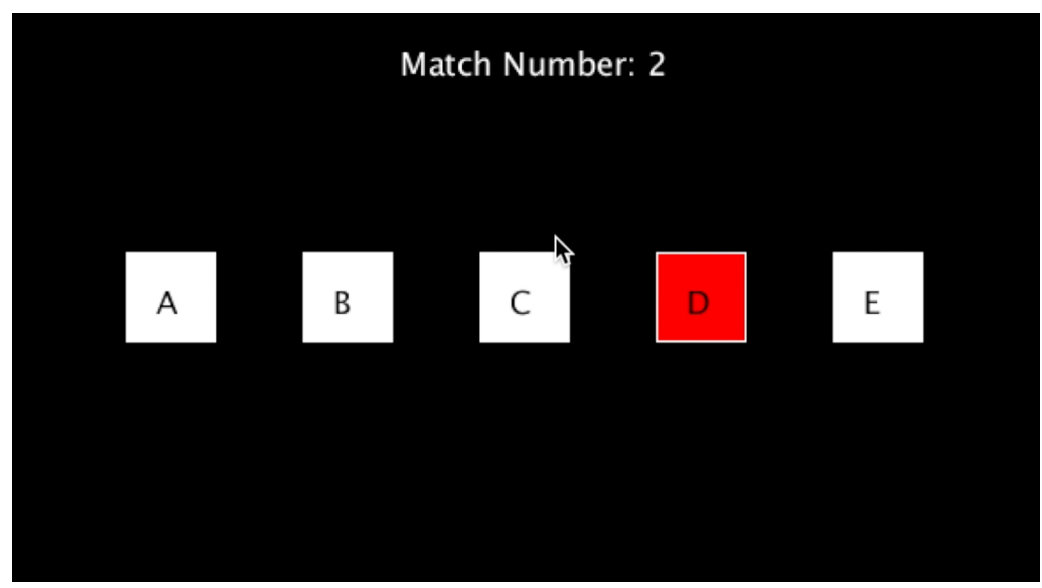

Figure 3.13: Screenshot of the box selection task. Here, the slider is positioned on box $\mathrm{D}$.

white background. Here, the output is dependent on brightness, and the brighter the screen the higher the value.

The light was kept in close proximity to the slider without being directly attached as to not obscure the screen during the study task. This is because the slider is acting as a second controller such as a smartphone or tablet. Therefore, the light output is not shown on the screen as this is acting as the item being controlled. In a scenario outside of the task the screen may take a different roll, such a Drone or RC car, and therefore the light output would be isolated to the controller in hand but still require attention on the item being controlled.

\section{Set-up}

The aim was to determine when engaged in tasks that require high visual perception, what output modalities can best provide secondary quantitative information via the cursor of tangible slider controls. We compare our four output modalities, which are shape changing, audio, vibration and light.

The set-up of the study (as seen in Figure 3.14) involves participants using our slider interface to control a custom application. This application is displayed on a 


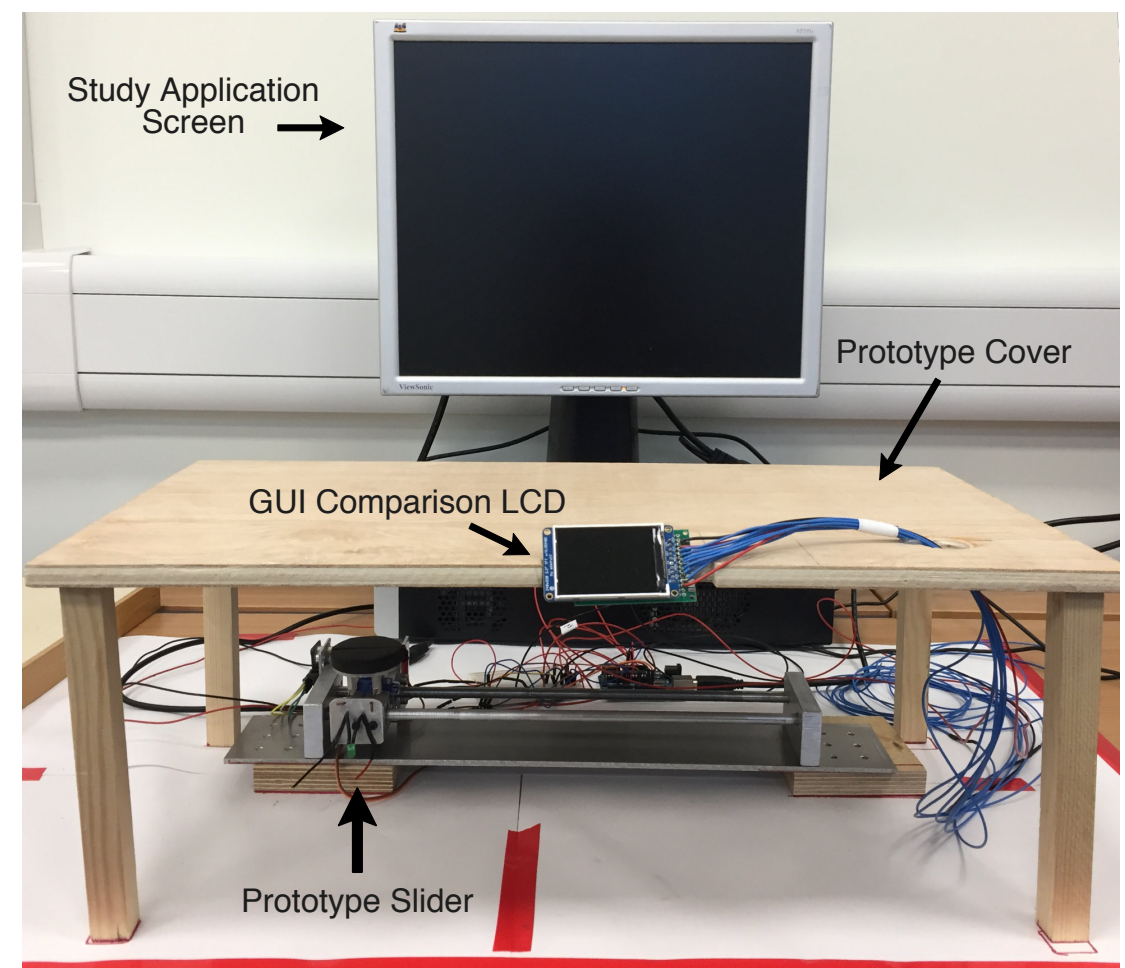

Figure 3.14: This figure shows a labelled diagram of the comparison study's set-ups from the perspective of how the user sits to the deck to use the prototype.

screen directly in front of the interface. Participants wore noise cancelling headphones throughout the study to maintain constancy with the sound output task and muffle the sound of the motors in the vibration and shape-changing segments. Participants sat at a desk to use the set-up and used their dominant hand to interact with the slider. The slider was hidden from view from the participant with a short table. The screen for the light output was placed on the front of the table in the periphery view of the participant.

\section{Task}

The task tested their ability to determine the points on the slider where the cursor was smallest and largest. After each task, they were asked to fill out a seven point 
Likert-scale questionnaire for visual attention, ease of use and enjoyment. Participants were asked to complete the tasks as quickly and as accurately as possible, but we did not impose a time limit.

On the screen, there were selection boxes labelled with letters (see Figure 3.14). By moving the slider, the participants moved the focus between the boxes. Each box represented a size that set the slider's cursor. Each box size was on a scale of 010 with no boxes in a single set being identical. The participants determined which box represented the largest and the smallest slider's cursor size and indicated this to the researcher. Once determined they pressed 'ctrl', which moved them to the next set of boxes. Users started this task with two boxes and were prompted with new boxes with an increment of one, up to six boxes. In total, five pairs of small and large selections were made. The screen in which the boxes were displayed on would require high visual attention through the task in order for the user to be aware of the box's label and to compare and contract what they could feel on the slider between the other boxes.

\subsubsection{Procedure}

Each participant started by filling out a demographics questionnaire. They were asked to perform the size determining tasks with the prototype followed by the Likert-scale questionnaire. We concluded with an interview about their experiences, where we discussed each output mode, asking for a rating of 1-7 and an overall rank order. We concluded with an interview about their experiences.

\section{Measures}

During the tasks, we logged users' time, accuracy and the number of switches between the boxes. To measure how eye-free the controls are, we video recorded the users. We also collected subjective measurements. We asked the users to fill 


\begin{tabular}{lcccccccc}
\hline Slider Mode & \multicolumn{2}{c}{ Shape Change } & \multicolumn{2}{c}{ Sound } & \multicolumn{2}{c}{ Vibration } & \multicolumn{2}{c}{ Light } \\
\hline & Average & SD & Average & SD & Average & SD & Average & SD \\
\hline Time (Seconds) & 14.12 & 10.10 & 12.74 & 6.69 & 15.67 & 9.74 & 17.87 & 10.64 \\
No. of Switches & 15.11 & 12.64 & 16.18 & 9.71 & 27.45 & 30.99 & 21.08 & 14.42 \\
\hline
\end{tabular}

Table 3.10: This table shows the average times and the number of switches for each output mode, including the Standard Deviation

(SD) for each one.

in a Likert-like questionnaire (1-7) based on ease of use, enjoyment and visual attention and the extent they could differentiate the values. After all the tasks were finished we asked participants to rank order the four modalities and give each one a rating (1-7). We also discussed their experience during the study in a concluding interview.

\subsubsection{Findings}

\section{Times and Number of Switches}

Table 3.10 shows the average times and number of switches needed for the participants to determine the largest and smallest values.

We used a repeated measures ANOVA to compare the time and the number of switches the participant need to the take before they had found the smallest and latest value in the set. We saw that the output mode had a statistically significant effect on the users' speed when picking out larger and smaller values using a slider $(F(3,253)=19.168, p<0.005)$. A post-hoc pairwise comparison indicated that this is where the significant differences lay. Sound was significantly faster than shape change $(p=0.029)$, and vibration $(p=0.000)$ and light $(p=0.000)$. We also saw that light was significantly slower than vibration $(p=0.038)$, shape change $(p=0.001)$ and sound $(p=0.000)$. 
40.00
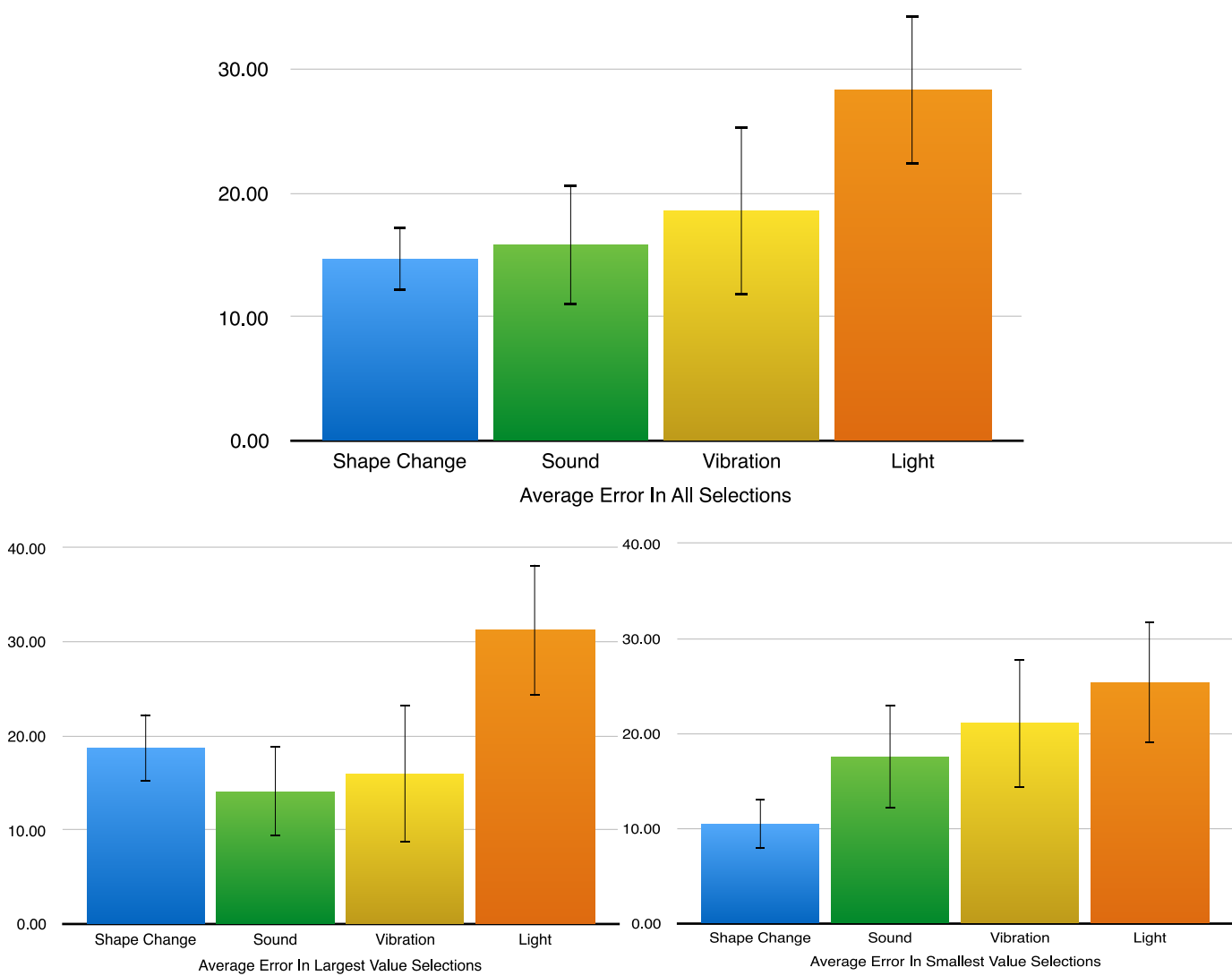

Figure 3.15: The percentage of incorrect guesses for each slider mode for the user selections. Top shows the overall percentages. Bottom left shows only guesses selecting the largest values. Bottom right shows only guesses selecting the smallest values. Error bars illustrate the standard error in each data set.

When testing the switches we saw that changing the output mode had a statistically significant effect on the number of switches between the values users needed to make before picking out larger and smaller values using a slider $(F(3,254)=$ 17.103, $p<0.005)$. A post-hoc pairwise comparison revealed that all pairs had a significant difference, excluding sound and shape change $(p=1.000)$. 


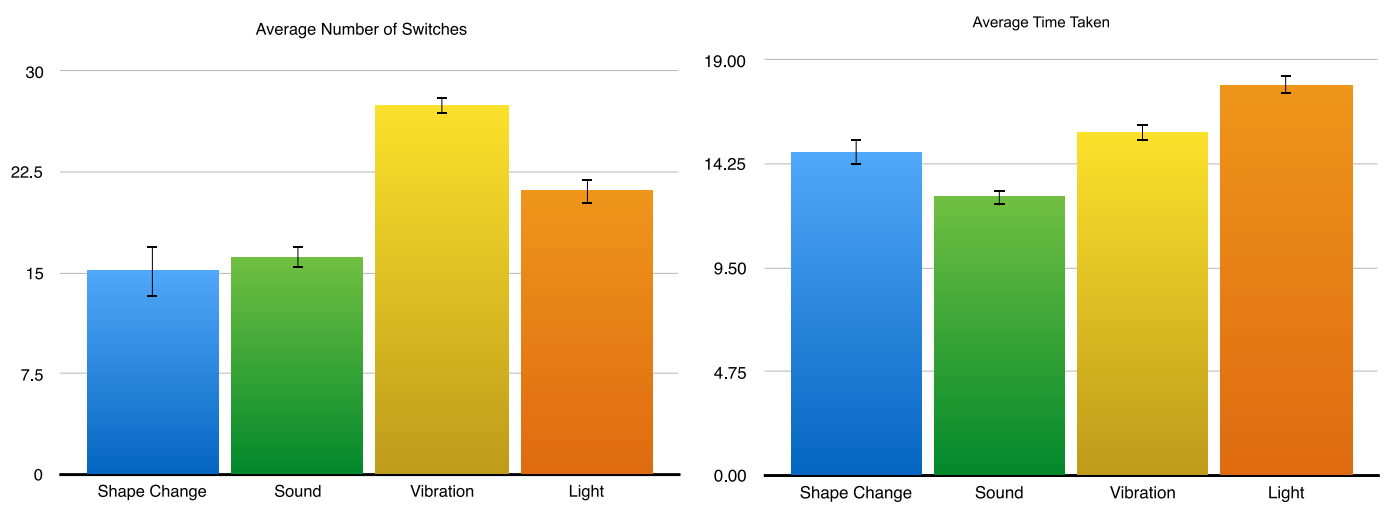

Figure 3.16: The graphs here show the average number of switches (Left) and the average time (seconds) taken to make selections (Right) for each output mode.

\section{Accuracy of selections}

Table 3.11 shows the percentage error averages of each mode for the overall sections and each small and large section. We used a repeated measures ANOVA to compare the error percentages to evaluate if the interface had an impact on the accuracy of the participants' selection.

Changing the output mode had a statistically significant effect on users' ability to pick out larger and smaller values using a slider $(F(3,29)=3.010, p<0.05)$. A post-hoc pairwise comparison indicated a shape change yield with significantly fewer error ratings than a light mode $(p=0.039)$. We also compared only the largest selections error; here, changing the output mode had no statistically significant effect on users' ability to pick out values $(F(3,13)=2.650, n . s$.$) . The same$ was seen when comparing the smallest sections error $(F(3,13)=1.780$, n.s. $)$.

We saw that participants' musical ability had a significant correlation with lower percentage errors in sound and shape change $(p<0.005)$ and also in light $(p<$ $0.05)$, while no significant correlation was seen using vibration. We also saw that participants with musical ability were more likely to rank the sound output at a higher rating. 


\begin{tabular}{lllllllll}
\hline Slider Mode & \multicolumn{2}{c}{ Shape Change } & \multicolumn{2}{c}{ Sound } & \multicolumn{2}{c}{ Vibration } & \multicolumn{2}{c}{ Light } \\
\hline & Average & SD & Average & SD & Average & SD & Average & SD \\
\hline Overall (\%) & 14.20 & 10.75 & 15.34 & 20.27 & 17.99 & 28.12 & 27.46 & 25.01 \\
Small (\%) & 10.55 & 10.88 & 17.58 & 22.27 & 21.09 & 27.09 & 25.39 & 26.27 \\
Large (\%) & 18.75 & 14.43 & 14.06 & 19.83 & 16.02 & 30.10 & 31.25 & 28.77 \\
\hline
\end{tabular}

Table 3.11: This table shows the percentage error averages and Standard Deviation (SD) of each mode for the overall sections and each small and large section

\section{Video Analysis}

For the video analysis, we watched back the video of each session and counted the number of times the participants' visual attention deviated from the monitor displaying the task, stoping the video to count each time. To mark this, we counted the number of times the participants' eye gaze would switch between the monitor and interfaces. We saw that the modes of vibration, sound, and shape changing required no switching of visual attention. When studying the video data of the users using the light interface we saw their attention switch 132.17 times between the screen providing the light output and the screen displaying the boxes in total across all trials.

\section{Experiment Questionnaires}

For our analysis of the Likert scale we used a Friedman test to test for overall effect. We then used a post-hoc Wilcoxon signed rank test, to evaluate individual differences. The summary for the questionnaires can be seen in Table 3.12.

There was a significant overall effect for question 1 , and the post-hoc analysis showed that light ranked significantly lower than sound $(Z=-2.898, p=0.004)$, vibration $(Z=-2.156, p=0.031)$ and shape change $(Z=-2.264, p=0.024)$. There was a significant overall effect for question 2 , and the post-hoc analysis again showed that light ranked significantly lower than sound $(Z=-2.736, p=0.006)$, 


\begin{tabular}{lllllll}
\hline & S.C. & Sound & Vibrate & Light & $x^{2}$ & $p$ \\
\hline 1- Extent you could differentiate values & 5.5 & 6 & 5 & 4.5 & 12.68 & $0.005^{*}$ \\
2- Visual attention given to slider & 1 & 1 & 1.5 & 5 & 13.68 & $0.003^{* *}$ \\
3- Extent you enjoyed using the slider & 5 & 5 & 5 & 4.5 & 16.38 & $0.001^{* *}$ \\
4- Extent the system was easy to use & 6 & 5 & 5 & 5 & 8.02 & $0.046^{*}$ \\
\hline
\end{tabular}

Table 3.12: It shows the statements the participants were asked to rate on a 7 point Likert scale (Low (1) up to High (7)). Groups with statistically significant effects are denoted with an asterix $(p<0.05$

(*) $p<0.005(* *))$. Shape Change (S.C.)

\begin{tabular}{lcccccccc}
\hline Slider Mode & \multicolumn{2}{c}{ Shape Changing } & \multicolumn{2}{c}{ Sound } & \multicolumn{2}{c}{ Vibration } & \multicolumn{2}{c}{ Light } \\
\hline & Average & SD & Average & SD & Average & SD & Average & SD \\
\hline Ranking & 5.9375 & 1.06 & 5.1875 & 1.68 & 5.25 & 1.48 & 4.3125 & 1.25 \\
Rating & 1.75 & 0.77 & 2.5 & 1.26 & 2.4375 & 0.96 & 3.3125 & 0.95 \\
\hline
\end{tabular}

Table 3.13: This table shows the mean values of the users rating for the output mode (1-7) and the mean ranks positions $1^{\text {st }}$ through to $4^{\text {th }}$. Adjacent is each Standard Deviation (SD) value.

vibration $(Z=-1.990, p=0.038)$ and shape change $(Z=-2.732, p=0.006)$. We also saw that sound ranked significantly lower than vibration $(Z=-2.070, p=$ 0.038). There was a significant overall effect for question 3 , and the post-hoc analysis showed that light ranked significantly lower than sound $(Z=-2.348, p=$ $0.019)$, vibration $(Z=-2.032, p=0.042)$ and shape change $(Z=-2.954, p=$ 0.003 ). There was a significant overall effect for question 4 , and the post-hoc analysis showed that light ranked significantly lower than sound $(Z=-1.997, p=$ $0.046)$.

\section{Ratings and Rankings}

We used a Chi-square test to determine a significance in participants' ranking of the output modes (see mean values in Table 3.13). The test showed that light was ranked significantly lower $\left(\chi^{2}(3)=9.50, p<0.05\right)$, while vibration: $\left(\chi^{2}(3)=\right.$ 
2.50,n.s. $)$, shape change: $\left(\chi^{2}(3)=7.500, n . s.\right)$ and sound: $\left(\chi^{2}(3)=1.00, n . s.\right)$ yielded no significance.

For our analysis of the interface ratings, we used a Friedman test to determine an overall effect. We then used a post-hoc Wilcoxon signed rank test to evaluate individual differences. Our Friedman test showed that was a significant overall effect $\left(\chi^{2}(3) 13.747, p<0.005\right)$. Our post-hoc test showed that shape change was ranked significantly higher than light mode $(Z=3.337, p<0.005, p=0.001)$.

\section{Interviews}

After the task, we conducted an interview with each participant. We discussed their experience with each of the four output modes and finished by asking about potential uses they saw. We transcribed all the audio recordings and performed a thematic analysis to identify shared opinions.

The first major theme we identified was the light mode not being eyes-free, with ten people bringing this up in some form. One stated that it was "quite difficult to use because of the split attention across the screen" and another said that "looking between the screen was a lot more clunky of a method". Furthermore, five of these participants explained how this affected their ability to select the values as they felt that this factor made it hard to differentiate the values.

Three of the participants described the sound output as annoying. One participant said that "sounds were effective but could be annoying" and another really did not like it, explaining that "it was in your head the whole time and it got confusing". One notable piece of information about the vibration came from a single participant explaining how they felt that the vibration was uncomfortable to use. They said that "[It] felt uncomfortable after a while; my hand still feels tingly". A participant also described the use of the shape changing interface as "very ergonomic to its purpose and so you could put your hand around it and it clearly felt the change in size". 
When we asked about future uses, six of the participants described its use for disabilities. They suggested that sound, vibrations or shape change could help both the visually and hearing impaired. Others saw the output modalities being useful in dark environments. One person described how the tactile modalities would be good in science museums. They explained that "science museums, where it gives a tactic response to engage people from different backgrounds easily about a common topic". Finally, one person thought that the shape-changing output could be used to engage children with learning disabilities, where they find learning through a physical easier.

\subsubsection{Discussion}

The results show there was a significant effect on users' accuracy across them experiencing the different output modes. The light is closest to a scenario with current technology, where users look down on a mobile screen to gather extra information. Yet our significant effect saw the new interaction modality shape change outperform in accuracy over light. The light did not only underperform in accuracy but also in subjective ratings. The light mode was ranked significantly lower against all the interfaces in questions 1-3 and worse than sound in 4. Also related to the accuracy results, shape change was the only interface to be rated significantly higher overall than the light. This suggests that it was not only more accurate, but that the shape change was also preferred by the users in most cases.

Finally, in the rankings, the light was also significantly more likely to be ranked the worst overall. Reasons for such low rankings surfaced in the interviews where people explained how looking between the screens made it difficult and harder to differentiate the values. It?s worth noting that the shape changing modes performance could be due it requiring less of a cognitive mapping compared to the vibration and sound and light due to it being physically represented. This would minimise some of the cognitive effort required by the participant. 
We also see that light and vibration would require more physical effort from users though operating the slider. Yet, shape change and sound mode would require a significantly fewer number of slider switches before the correct answers were determined. On average, the participants were faster using the sound output. This could have been due to sound switches being quicker within the system influencing the time it may take. Shape change takes longer to actuate into its position and with $(p<0.05)$ compared the instant output of vibration and light was a very viable competitor to sound.

Sound results were also affected by the participants' musical ability so the more accurate results would have been improved by a subset of those taking part. This tells us that it may not fit all users as not everyone has musical talent. Another limiting factor of sound is its need to play constantly, avoiding users forgetting or missing played value. It would also make the user's environment noise, making it hard for communication with others - and vice versa if the environment had preexisting noise, as they would not hear the slider output. Participants even voiced in the interview that the sound was annoying. These factors would not be an issue in the shape-changing mode. So even though the sound is cheap and deployable now there are other conflicting arguments for it not being suitable for all use cases.

Overall, the results show that a shape-changing modality on sliders could be a viable option to deliver extra contextual information on sliders in eyes-free scenarios. We see that it outperformed current dual screens graphical outputs and provided accuracy and a good user experience. For the artist or designer this benefit would aid in helping them focus on their canvas rather than switching to look at GUI controls. We also see that the modality of shape changing holds possible advantages over vibration, which required more operation of the slider from the users. Sound output was still the quickest method but may not be as effective in scenarios where outside sound is critical or overriding the slider sounds. It would even distract the user from their task at hand, which is no good for an artist in deep flow. Furthermore, we 
also saw the possible limitations of users requiring musical ability to perform better when using it. This leads us to consider that shape changing cursors may hold potential benefits over other output modalities, and therefore could open a new output modality for designers and researchers to explore when building new interfaces.

\subsection{Future Sensels for Art Tools}

The prototypes and studies in this chapter have contributed to the sensel-like displays and controls, and in this section, we draw some comparisons and lessons from the two studies. In both prototypes, there is some form of deformable output that physically indicates the system's state. In the first system, this is done via the changing form of the tools to indicate the task (in our prototype this was nib and colour selections). However, they both present unique lessons for future work in the space. The finding shows how the first deformable interface capable of emulating tools and switching between them proved to be an enjoyable experience, but currently, the system is limited by accuracy as the force controls used in the version of the prototype we developed.

The prototypes' use of deformable output while using the a slider resulted in this holding some benefits over other output modalities and opened a new output modality for designers and researchers to explore when building new interfaces. We illustrate how an example of this could be utilised in future sensel-based controls in Figure 3.17. This depicts what a sensel with actuated feedback might look like in future, combining elements of the prototypes from both studies. In this way, the artist would gain the benefits of actuated tool switching, while also feeling the size of a tool, such as a nib. 


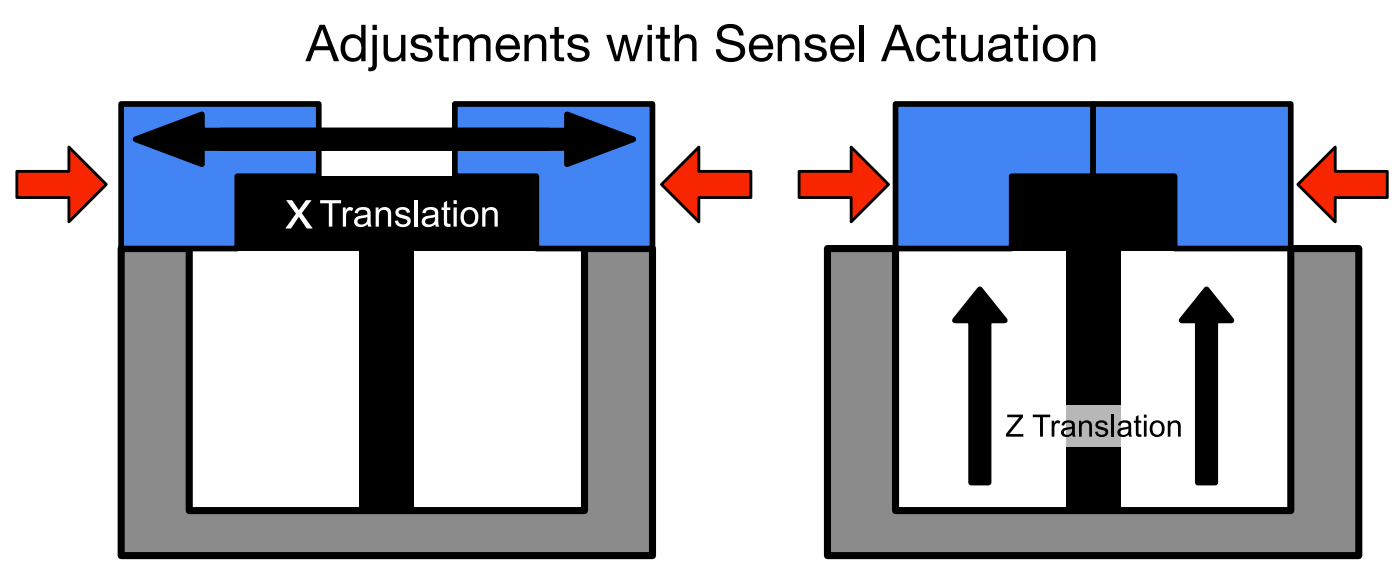

Figure 3.17: Returning to the figure from Section 3.2.1 we illustrate how actuation as shown in the slider study could be added to sensels as seen in the first prototype.

\subsection{Summary}

This chapter explored two types of interfaces that employ actuated elements of deformable interfaces to physically represent tools used by artists. We demonstrated how to develop interfaces that begin to mimic realistic elements of painting and art creation with respect to nibs, paint pots, and sliders, and presented evaluations of each system.

In the first part of this chapter, our work demonstrated how we can use actuated sensels to provide dynamic force-based controls in a digital painting scenario. We concluded that participants are not able to use our deformable prototype as accurately or as quickly when performing digital painting tasks. Despite this, many of the participants enjoyed using the new interface. In interviews, they highlighted the advantages they saw in it and future uses.

The second part of this chapter presented a follow-up study that shows how we can make elements of digital art interfaces physical. This study introduced the concept of concurrent interactions and deformable controls. We have shown an example of a prototype built to illustrate how the courses of physical sliders can be deformable while in use. We then documented how this performed in a user study, 
where we compared the use of the slider to different concurrent output modalities. Overall, the results showed us a shape changing modality output for sliders, which could be a viable option to deliver extra contextual information on sliders in eyesfree scenarios. We see that it outperforms current dual screens graphical outputs and provides accuracy and a good user experience.

Overall, this chapter has shown the capability for deformable interfaces to increase the level of engagement and enjoyment for the user compared to their graphical counterparts. This now provides us with questions surrounding how well we imitate these physical interactions and how close we are compared to what really artists do. In the next chapter, we conduct a study with artists to better understand what makes art a physical experience. We then extend our knowledge of interaction capabilities from mechanical actuation and force input to textured actuation that feels like the materials seen in the initial study.

\subsection{Authors Contributions}

The concept and implementation behind this research was the authors. Ideas were discussed and critiqued by the authors $\mathrm{PhD}$ supervisors. The design and development of the prototype was solely done by the author. The author also planned and ran the study sessions, analysed the results. The supporting paper was written by the author with feedback from secondary authors. 


\section{Chapter 4}

\section{Dynamic Paint Textures}

In the last chapter, we introduced the concept of using deformable elements of interfaces to simulate tools and present output modalities. These ideas provide us with the foundations for this next study, and as in the previous chapter, we looked at how deformable interfaces can represent physical tools such as pots, nibs, using interfaces with actuators and sliders.

In this chapter, we set out with an aim to discover what tools and techniques painters use that are not yet simulated in the digital world and were important qualities to the artist possibly missed or prevented digital offering them a complete experience. We aimed to focus on an element of the painting experience that deformable interfaces could aid in bringing such an experience to the digital world. Therefore, our goal was threefold, first, we would observe painters practising their art and interview them about their processes in order to then, secondly, once are we had suitable findings and found an element of painting with which to explore, we would build a prototype that would best simulate this experience before finally planning to use the prototype in a series user studies to test how well it simulated the chosen element of painting identified in the study.

We demonstrated how the use of input such as force can attempt to simulate the sensations of dipping and picking up paints. Furthermore, by adding foam to the interface as a texture, we further support this interaction and provide users with a 
textured feeling when using the interfaces. Then in the second slider prototype, we studied how we could hold these shapes via actuation of the slider cursor, so that unlike the foam, the interface would not decompress after the user let go.

In this chapter, we build on the exploration of how to build richer interactions for digital art tools by taking a deeper exploration into the textures of art and how to imitate them in digital interfaces through the use of synthetic chemicals. We began this chapter by looking at the different tools and materials used by painters in their art processes via direct observations and interviews. We contribute to the documentation of these findings and how this research allowed us to identify the importance of texture and how it relates to mixing paint on palettes. This study gave us the findings to support the development of a deformable interface, furthering the literature on dynamic textures by simulating interactions with paints via the use of hydrogel. This material demonstrated actuation possibilities for the back of device interaction on mobile screens for simulating soft textures and providing gestures such as mixing and stirring.

We evaluated the prototype with the artists from the original observations. Their feedback claimed its similarity to oil and acrylic paints and a palette experiences with regards to starting with blobs of paints and then using mixing gestures to soften the paint when mixing. Insights of this work with the artists and development of the prototype were presented as a paper in MobileHCI 2018 [110]. We then conducted a further lab-based study to evaluate the gel's similarity to paint in more controlled experiments and gathered a further understanding of participants' options when using the system. This study showed a significant similarity to oil paint and presented further findings to support its affordance for paint palette gestures.

These insights have built upon the previous chapter on colour pots, and focuses on the role of colour mixing on palettes and the use of dynamic textures. In doing this, we explore the viscosity in paints for stirring and spreading on the palette in order to mix the desired colours. Using the prototype, we present the qualitative 
results of the artist's experience with the prototype, along with a controlled study evaluating user perception of the gel compared to paints. We conclude the chapter by explaining the implications that texture in a deformable interface can have, along with the possible future uses of the gel in interface design.

\subsection{Introduction}

Digital painting tools have become a common form of expression for many artists, and provide many capabilities that often surpass the limitations of their physical counterparts. Despite these enhancements, however, many key physical painting features are not emulated digitally. One such example is the lack of textural feedback that is provided when mixing different viscosities of paint; a key action that aids artists in achieving the correct dilution, and hence, colour, of their medium. In this chapter, we specifically explore the lack of tangibility in digital painting with regard to the lack of texture when it comes to mixing paints. These elements play a key role in physical painting and expression of colour, so we developed tools that combine elements of physical painting with digital painting.

Stemming from direct observations and interviews with both physical and digital artists, we saw an opportunity to develop a hybrid interface that combines the benefits of physical paint to provide the digital artist with a programmable, textured surface for colour mixing. This is designed to be used as an input palette on the back of standard hand-held mobile devices, giving the artist the ability to feel the texture of paint with their fingers on one side, whilst seeing the effect of the paint's colour on the other. Our novel interface uses hydrogel [73], a chemical substance that changes its stiffness based on temperature, allowing us to manipulate the apparent viscosity of the palette to give user feedback while mixing paint.

In this chapter, we discuss a series of observations and interviews we undertook with both physical and digital artists. We describe how this informed our design 
and detail the implementation and capabilities of our prototype. Our prototype includes both heating and cooling components to allow us to directly manipulate the viscosity of the gel palette based on user behaviour. While providing feedback in this form, the gel palette also acts as an input, detecting motion and altering the colour/dilution of the front display accordingly. Artists interact with the gel using their fingers - which are powerful sensory receptors - which enables them to mix digital colours with realistic texture sensations but without mess. We document the design process and the capabilities of this dynamic paint-textured prototype. We then discuss the feedback gathered from the artists when we return to show the prototype. We finally discuss the artist's feedback on the prototype and at a lab-based study exploring the gel's similarity to common paints.

\subsection{Observing Artists}

\subsubsection{Method}

We wanted to discover elements of traditional painting that would inspire the development of textures and materials for new tangible user interfaces for creating digital art. To achieve this aim, we went along to local art group sessions to observe how people paint. We did this with members of a local community art class in Swansea. This was a group of people who meet to conduct their art individually in a shared environment. Within the group, they teach each other and plan exhibits to sell and showcase their work. We observed and interviewed seven artists at the classes, two of whom whose focus was sketching and five who focused on painting. The members' ages ranged from 46 to 72 and all had been practising their art for over 10 years.

During the art class, we observed and recorded how they created their art and asked questions about their artistic process. After this, we retold our observations 
and conclusions back to them, in order to confirm our observation's validity. This allowed them the opportunity to input into clearing up any misconceptions or to enlarge important points. Information was collected via note taking, photography, video recording and audio recording.

Alongside these artists, we worked with an artist who practiced both digital and traditional painting techniques professionally. His work included both commission work for big brand super hero movies and his own works in a shop and his own market stalls. He has also taught art in schools up to A-level qualification standard. We reached out for an interview with him after meeting at his own market stall. This aided our understanding of how traditional techniques are emulated in existing digital art applications and how it changes the paint mixing workflow. In the interview, we discussed the existing tools available to digital artists, the benefits of digital, the tangibility he misses and his workflow for selecting and mixing colours.

\subsubsection{Findings}

The artists had lots of different tools that they used to create their art. First, we discussed their different brushes. The painters would use lots of different brushes simultaneously while painting (see Figure 4.1). This was to take advantage of the different sizes and stiffness. One of the painters explained that it was not only the size of a brush but also its stiffness, as this allows them to bend the bristles on the canvas for a larger or smaller spread of paint. One of the artists also manipulated their brushes. She cut an old worn out brush to use for a realistic fur effect in her animal painting. We also saw them use two of the same brushes only with different colours. They held the other brushes in-between their other fingers and used different colours on them.

The artists also worked with unique tools to create different painting effects. They explained how they manipulate the bristles of a toothbrush by squeezing and 

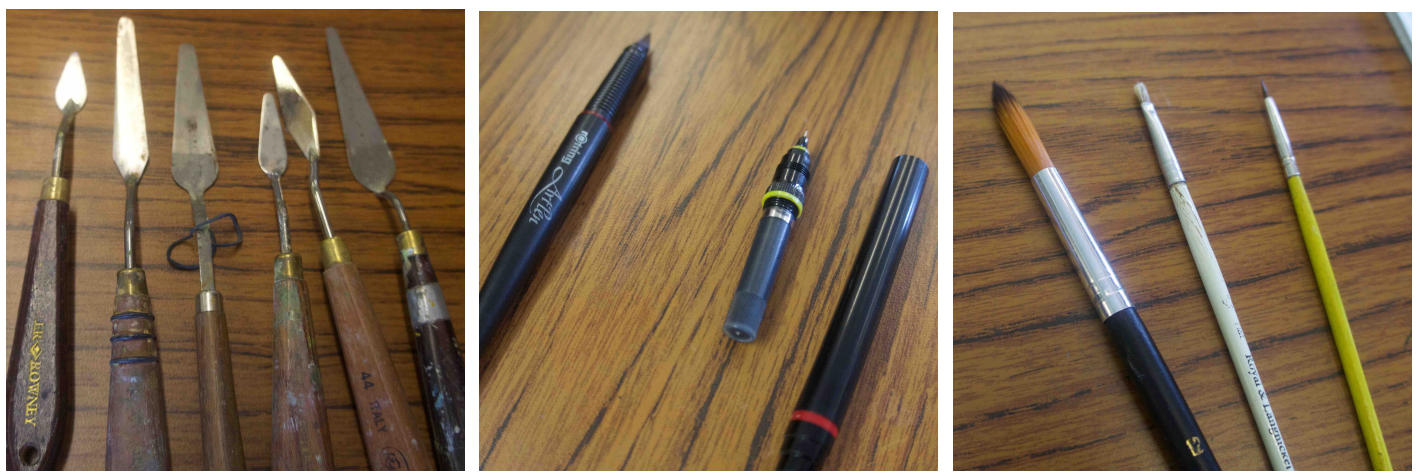

Figure 4.1: Painting Tools: Artist knifes (left), Artist Pens with interchangeable nib sizes (centre), Brushes: these three different brushes were being used simultaneously by and artist (right)

flicking to transfer the paint onto canvas. Another tool one of the artist used was knifes (see Figure 4.1). They used these to spread and work in the paint and specifically used them to create raised out of painting areas. We saw an example where one artist was painting blossoms and using this effect (see Figure 4.2). Another tool they use is sponges, which an artist was using to dab out trees.

The sketchers mainly used pencils and pens. One of them would occasionally use black washes and watercolour pencils. These colours would be applied via the pencil and then water was added to blend the colours in. This sketcher draws when out walking and tries to quickly capture the sketches while on the move. They would leave notes for what they did not capture to draw and colour in later. When they are out, they only take a couple of pens and pencils.

The other sketcher drew only black outline drawings. They explained how they were colour blind so using colour was difficult. This artist used pens with switchable nibs, which allowed him to use the same pen handle after changing the nib.

During the sessions with traditional artists, we observed them mixing and creating their colours by dragging, scooping and stirring paint around around artist palettes. When we were with the painters, we saw that they used watercolour paints, oil paints and acrylic paints. Water paints are very thin with a watery consistency 


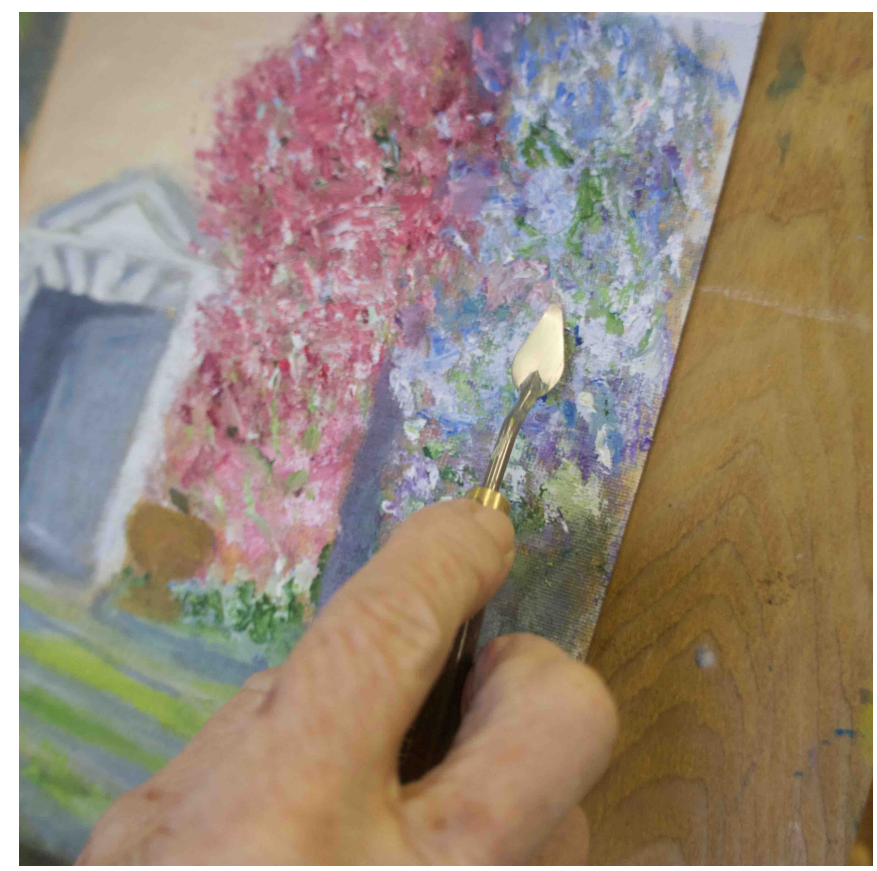

Figure 4.2: Artist using painting knifes to create raised areas in painting. They do this by scooping up areas or layering on thicker amounts of paint. The image here show an oil painting.

and are light in colour. The artist we observed using them would wet their brush and then rub this against the block of paint until the water took on the colour. They then dragged this coloured water to their palette, and from there, they dipped their brush to use that colour in their painting. When they mixed multiple colours they would do this for each colour and then begin dragging from each watercolour source to make their new colour. If they needed the colour to be brighter, they would add more water to it. If they needed the colour to be darker, they would add more coloured water from the source.

The oil paints are described as a lot thicker to work with and are naturally darker in colour. We observed that the artists would squeeze out a selection of colours onto their palette from the tubes the paints were supplied in. From these squeezed out colours, they drag and mix the paint to different sections of the palette taking a bit of paint from each of the blobs squeezed out. They add white paint or a lighter version of the colour to make the colour brighter. They do this on the palette or in 


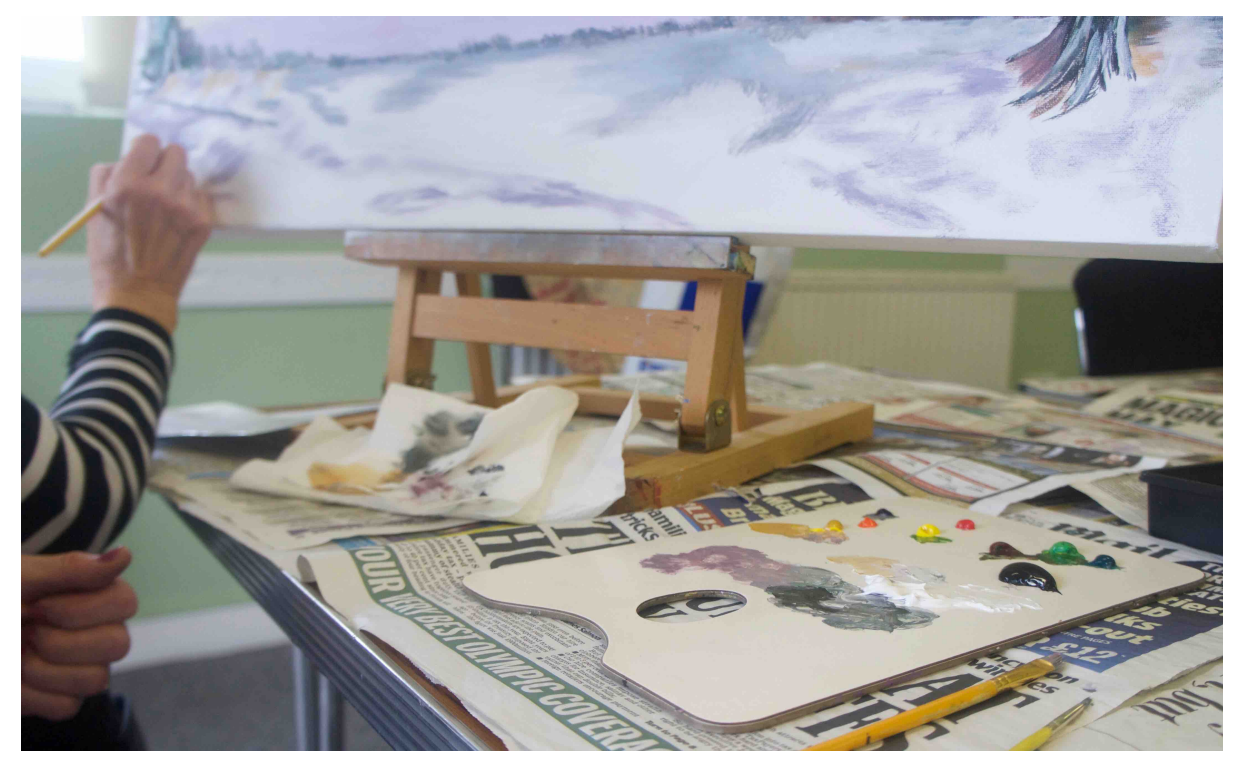

Figure 4.3: Artist using palette for paint mixing before taking the paint to canvas. Notice how the palette colour matches the blends of colour on the canvas. The palette gives a history of the colours used in their painting.

highlights in the painting on the canvas. When working with oils, one of the artists explained that they need to scoop a lot on and work it into the canvas as it absorbed quickly. They described that using the acrylic paints felt like a mix of the two paint types. The process of mixing we saw resembled the oils but the textures and feel of the paints were more watery. Other miscellaneous materials used were builder's cork/wood filler. This was used to create raised effects in the painting. The artist then painted over it once it was dry.

All the painters mix their paints out onto palettes before applying the paint to canvas (see Figure. 4.3). They start with a knowledge of which colours mix to make a certain colour. These are the colours they start with on their palette. Then, using trial and error, they go through stages of adding a bit of each of the colours to different areas of the palette. During this process the amount of paint scooped would determine the tone of the colour. The stages their different colours mixes would go through were spread across the palette. This showed them a history of colours mixed and allowed them to return to the colours at any time during painting 

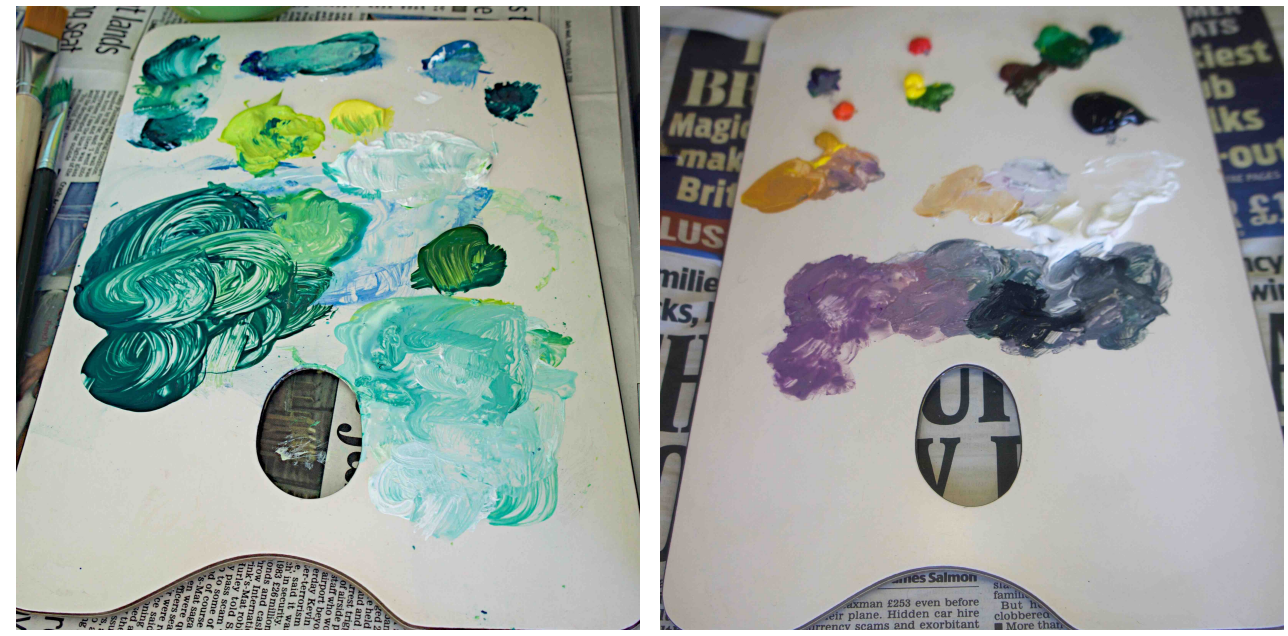

Figure 4.4: The mixed colours left on a palette after painting. This shows the history of the colours mixed for the painting

(see Figure. 4.4). As part of their process, they put the paint in certain locations to remember where they were. Another interesting thing we noticed was the artist sometimes left a percentage of the paint unmixed. This, for example, will give spots of the whole unmixed colour within a different colour. Once the artists have their colour, they try a little bit in the corner of the canvas as they said it will often look different in the canvas compared to the palette, so they need to test it first.

\section{Digital Art}

In our interview with the digital artist, he opened the discussion by explaining what he found to be the major benefits to digital painting. First was the eraser, and he demonstrated on this app how it helps him achieve sharp edges. The other he pointed out was manipulating the canvas. He explained that it is very important to create distance between you and your painting. Digital apps make it easy to zoom out.

When we asked about his workflow for colour mixing, he explained that he picks his colour values according to a scale e.g. 0-10 for shades. This allows him to the create $3 \mathrm{D}$ form based on lighting in the scene. He lays these colours out 
onto a physical palette and adds/removes white to go up or down across his scale. He emulates this process in digital, but instead of a physical palette he mixes the colours on an additional layer in Photoshop (see figure 4.5). He uses the pipetting tool to set colours actively. He then uses the blend tool or smudge tool to mix digital colours. This is done by layering dark to light and then smudging the edges. He commented how "it looks great but you're not getting that feeling of mixing". He also noted that other artists may build up layers using the opacity tool, though he has never tried this.

He was very clear that about digital lacks a tactile feel. No matter what tools he's tries everything feels like a screen. He spoke of the drag factor on his palette and sketchbooks. He said how everything has a different feel to paint and draw on, from canvas to tonal paper, even cardboard. He mentioned that he prefers the drag when he works. Also of note, he said "I don't feel like it's finished until I have a hard copy. With digital work, I love it when it's on the screen but I don't feel as if its finished until I've got a hard copy." He said that this was especially important when working with a client as he felt that it relates to their value for money. We also discussed the possibility of mobile digital art. He personally does not regularly use a mobile device for art due to limitations such as screen glare, battery life and screen heat. Though despite this, he identified that a tablet would weigh less by the time he packs his pencil case and sketchpad.

\subsubsection{Discussion}

A large take away from our study was the role of physical gestures for mixing colours. The way artists interact with their palette is different to modern painting applications. There are a lot of physical gestures and textures when mixing paint on the palette before it taken to the canvas. Key physical gestures such as dragging, scooping and stirring play a large part in colour section. This is absent from the digital experience. We saw that physically dragging the colours around the palette 

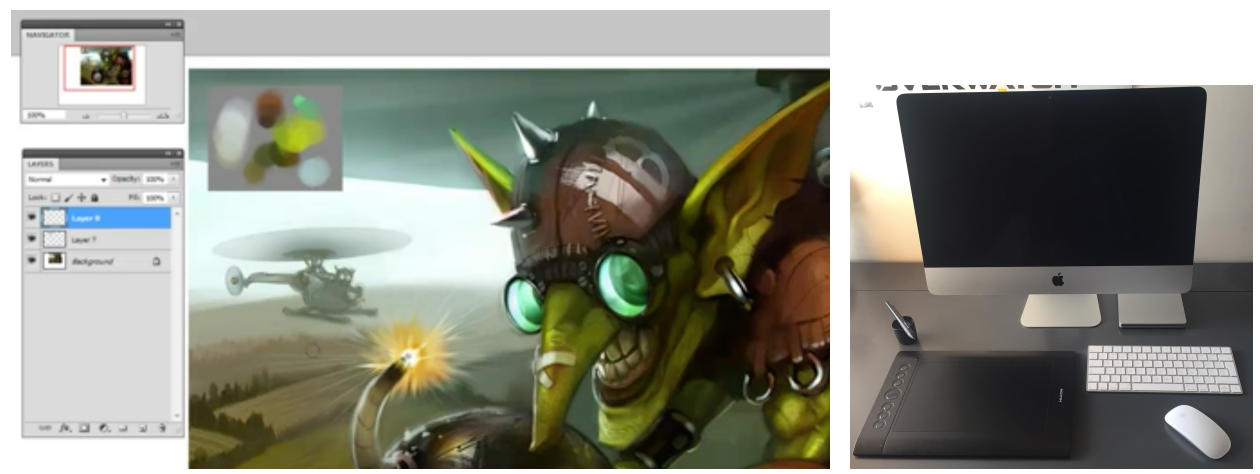

Figure 4.5: Left figure shows how digital artists will add an extra layer when they're painting in Photoshop for mixing paint. They will hide and reveal this layer as needed. Right figure shows the digital artist's work station.

allows them to maintain their different shades. It also gave artists a spatial knowledge of where the different colours lay, allowing them to maintain flow to their work.

Another aspect to this was colour saturation, as the amount of paint scooped determines the amount that is brought over on the brush or knife. The amount of physical stirring gave artists real fine control over how much they mixed. Part of this then is the layout of the colours. We saw that the palettes had a certain order where colours have organically developed. You could see their evolution through the many shades trailed across the palettes. This gave a sense of physical history to the colour section process. We also learnt from the digital artist how this physical paint mixing process is emulated in digital, but that he missed the tangible elements. Despite missing these elements, he shared how digital offers different benefits for his workflow. This will inform how the two worlds can be combined to offer both tangible and digital benefits.

Overall, these findings showed us what are the physical aspects of painting regarding the colour mixing the feel of paints. Alongside this we also see the important role physical feeling played in painting for the artist in the class and then again with the description from the digital art and he missed the tangible experiences so 
much so he would use physical mediums before switching to digital. This highlighted to us that the realistic experience of the painting materials was important to the artists and something completely missing from digital. Therefore, it is these key findings that informed the next sections, were we describe the prototype we built aiming to emulate this possess for digital art and create realistic artist interactions for both feel of paint viscosity and the flow of mixing colours on a palette.

\subsection{Prototype Design and Development}

Based on the observations reported, we began ideation around a scenario of physical digital painting. This would pair the many benefits of digital painting without losing the physical sensations experienced when painting physically. It aims to offer a seamless interaction between physical materials and digital information. After seeing the important role the physicality of paint played in the artists' creative process and the joy it instills, we wanted screens to be able to transform into paint-like material, as the physical feel of paint is absent from today's digital painting tools. To begin to understand how it could be introduced, we imagined a scenario where areas of a screen could have varying viscosity representing different paints. This would allow users to dip, push and swirl around areas of the screen like a painter's palette. To aid in the realisation of this, we undertook designing and developing an early prototype that showed materials that could offer the beginning of a physical digital painting. The prototype aimed to deliver textured paint mixing sensations, support for stirring paint using the textured material, the separation of palette and canvas in a mobile system pairing mobile phone, and tablet devices.

To achieve these goals, we explored the use of adding contained hydrogel to the back of a mobile smartphone, with the goal of providing tangible colour mixing that fits in the palm of the artist's hand. This layer of viscous material can be used for haptic colour mixing, simulating the physical sensations of paint. Hydrogel 

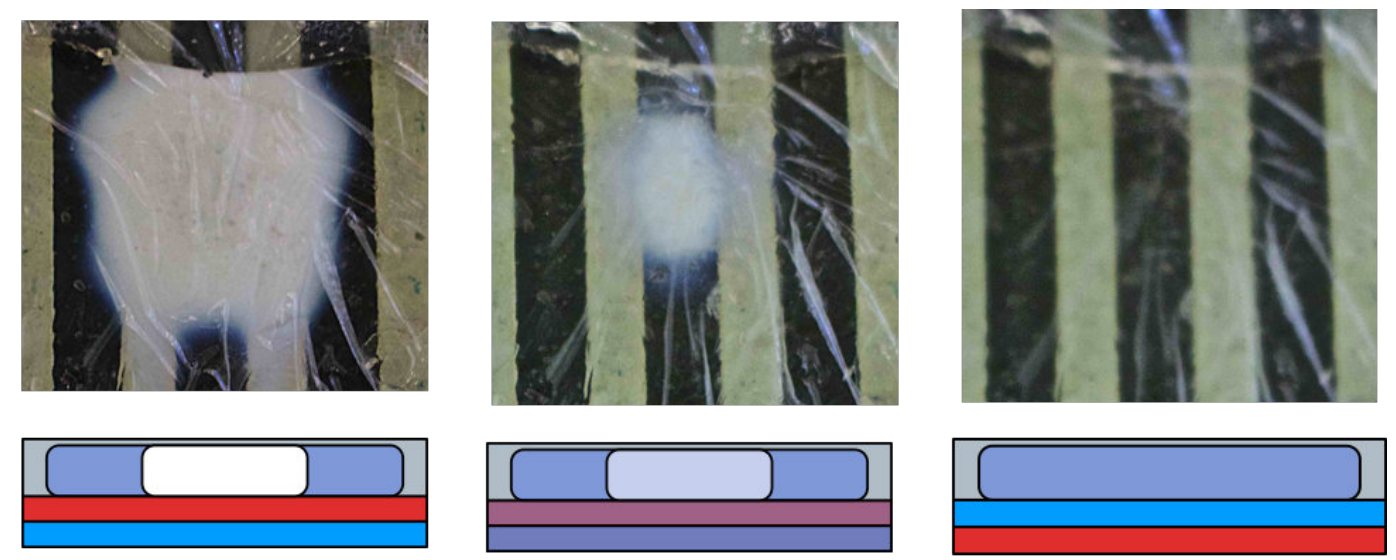

\section{Temperature Change}

Figure 4.6: Hydrogel states as temperature is changed, Left: The gel is at its hardest, feeling like an oil-based paint. Centre: The gel is softer and thinned out, feeling more like an acrylic-based paint. Right: The gel is now at its softest after being fully thinned out, and feels more like a water-based paint.

can actuate from stiff to soft, providing artists with a tangible sensation of thinning paint for different shades of colour. The output of the colours are seen on the phone application. From here, the artist can pick the colour and paint on their canvas. We provided a canvas application on an iPad, which was then paired with the tangible palette phone for transferring colours.

\subsubsection{Hydrogel Paint}

As one of our key goals was to simulate the sensation of paint, we needed to find a material near the viscosity of paint that could be manipulated by both the computer and the user. We experimented with hydrogel, a soft and viscous chemical with similar properties to those of paints. It also has the ability to stiffen when heated. This means that we can actuate the material via stimuli triggered by the computer. This offers us different levels of stiffness to simulate different kinds of paint depending on the user's desired interaction. The actuation works by first heating the gel to a 


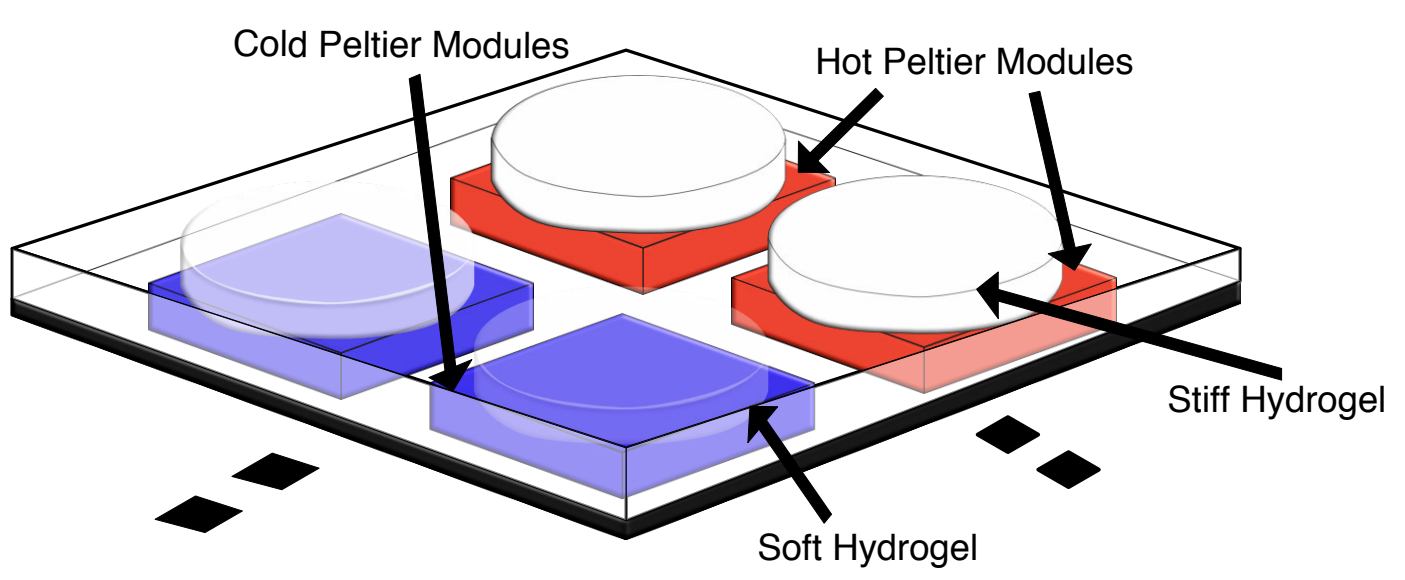

Figure 4.7: This figure shows a transparent view of the prototype. Each Peltier module is set under the gel. The red Peltier module shows stiff gel in a fully white state and the blue Peltier shows soft gel in a transparent state.

stiff state. When cooled, the gel softens. This variability can then be mapped to the stirring and thinning out of paint. We also saw this variability as an opportunity to simulate different types of paints such as oils, acrylic and water paints.

After testing that the hydrogel had the potential to simulate paints within a lab setting, we began the technical challenge of changing the temperature on demand paired with a graphical output. In our initial test, we used a resistance wire that ran through the gel and was heated using a power supply. While this worked well in heating the gel, we needed a process for cooling the gel as quickly as we were heating it, to provide the most natural interaction for the artists. We also needed this process to be reversible, so that wherever the gel was placed, it needed not only to heat but also cool the gel. We accomplished this by using a Peltier module. These produce thermoelectric heating and cooling when current flows through them. During the process, one of the sides will heat up while the other will cool down. If the current flow is reversed, the opposite sides will heat and cool. This means that we can switch the temperature programmatically using $\mathrm{H}$-Bridges paired with an Arduino, making the stiff to soft actuation programmable in an interactive system. Now that we had this set up, when we switched the hot and cold sides the hot side still took too long to cool, which limited the instant effect on the gel. We also 


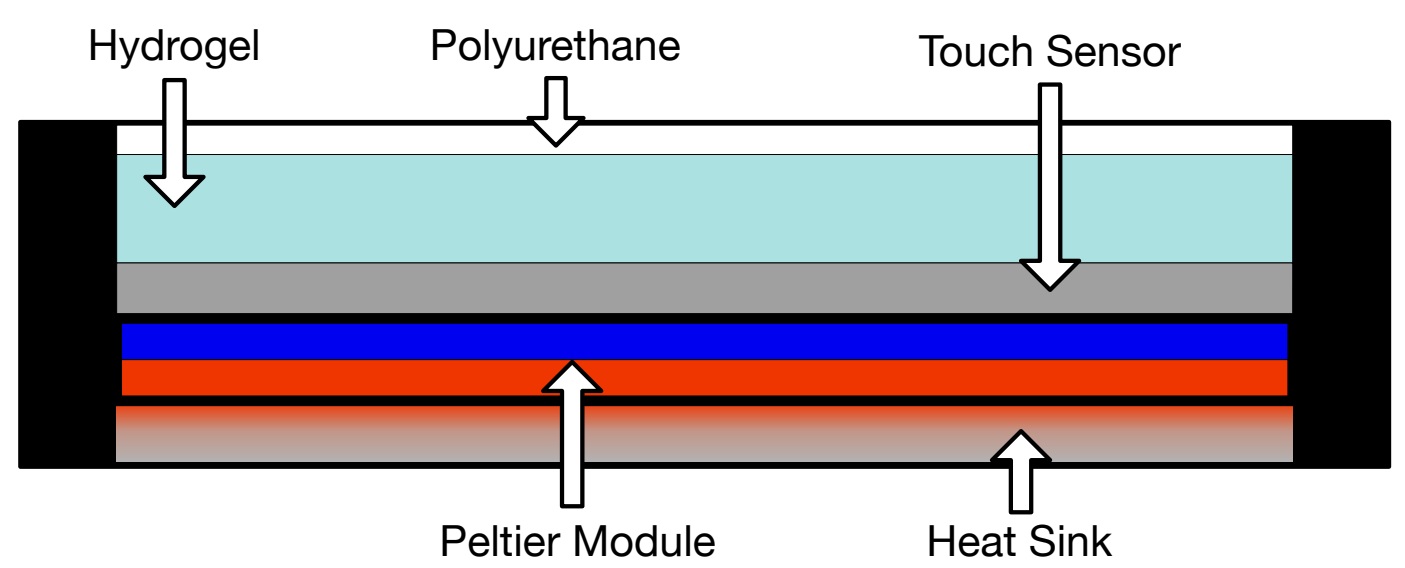

Figure 4.8: This figure illustrates the layers of the prototype used to activate the gel. Here, a Peltier module is set to cool the gel (blue side).

added a heat sink to the side of the Peltier opposite the gel, which aided cooling and speeded up the process of state switching. This aided the cooling, as the more heat that can be dispersed, the faster the opposite side can be cooled. How the temperature change effects the prototype can be seen in Figure 4.7.

To produce our hydrogel we prepared it following the methods presented in the Gel Touch paper [73] by Miruchna et al. First we dissolved N-isopropylacrylamide in distilled water then added N,N?-Methylene-bis-acrylamide to the solution and dissolved it using a magnetic vortex. We then put the solution on ice for 10 minutes while we dissolved Ammonium Persulfate (APS) in distilled water. We then missed these two spoliations into the beaker to then add the TEMED as an accelerator for the chemical reaction taking place. We then poured this final solution into the phone case, where we left it to set in the fridge overnight. Then when placed on the Peltier array seen in Figure 4.7 the gel would activate to its stiff state when reaching a temperature of approximately $32^{\circ} \mathrm{C}$ when heating the Peltier device. Then as we cooled the gel with the Peltier reaching approximately $4{ }^{\circ} \mathrm{C}$ the gel would switch to soft state. 


\subsubsection{Combining with a Mobile Phone}

In order to provide interaction with paint, the system needs to detect the location and pressure of the user's interactions with the gel. We achieved this by placing the gel on a touch-sensitive layer of Velostat with a grid of conductive tracks that allowed us to detect touches at intersecting points. This is also capable of sensing the force of the user's touch, which allowed use to map how quickly the gel should soften.

Having provided both sensing and actuation, we then needed a way to display the colours the users would be mixing. One limitation of hydrogel is that it becomes opaque when stiffened, obstructing whatever is behind. This limited the possibility of putting the gel over the screen as colours would only be visible when the gel was is in the softest state. This prompted us to put the graphical output on the front of the device, and the hydrogel on the back. Doing this supported the interaction of holding the mobile in the non-dominant hand to mix colours while using the other hand for painting, resulting in a palette and easel set-up as seen in our artist observations (see Figure 4.10).

\subsubsection{Final Interaction}

The final interaction of the prototype allowed the user to hold the device in a single hand with their fingers on the back to reach the gel, while their thumb on the front screen is able to select the desired starting colour (see Figure 4.11). It also moves us towards envisioning the gel palette in the form of a mobile accessory that can be added to any standard mobile device.

The user begins their interaction by picking the colour on the phone app (see Figure 4.9). This imitates how an artist would pick out their starting paints to place on the palette. Once the colour is set, the gel on the back of the phone can now be manipulated, as touch sensing and temperature control in that area is now active. 

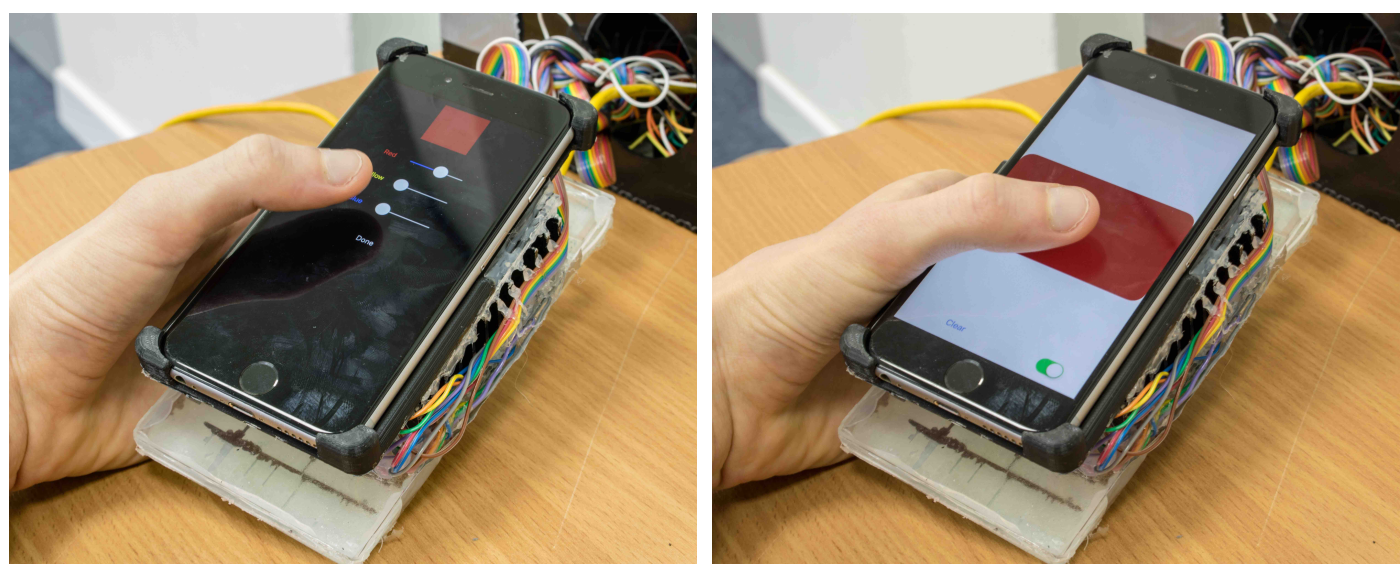

Figure 4.9: The GUI displayed on the phone. On the left is the menu to select the initial colour. On the right, we see that colour is now in the centre pot, which activates the gel in that area on the back.

When the user touches the back of the mirrored location of the paint on the screen, the touch is registered as an interaction. From this point, the user can push and rub the gel. This will cause the gel to be actuated towards a softer state. These stages of actuation are illustrated in Figure 4.6. Simultaneous to this, the user will see the colour on the phone screen decrease in saturation. This interaction process simulates the paints to thin in order to alter their shade. Once the user has mixed their desired saturation, they can select it with their finger or stylus on the phone screen. They then use the colour on the canvas application.

Our technical implementation of the prototype is layered to provide the temperature actuation (see Figure 4.8). The hydrogel sits under a layer of polyurethane on top of the resistive touchscreen. This screen then sits on a $4 \times 3$ array of Peltier modules, each with a $15 \times 15 \mathrm{~mm}$ activation layer. Each Peltier module can cool or heat the gel. This is controlled by an IC chip, so by using an Arduino, we can switch the H-Bridge within the chip for each Peltier to either cool the gel making it appear soft or heat it to appear stiff. In order for the Peltier to efficiently cool the hydrogel, we add a heat sink underneath the grid. This disperses the heat from the hot side of the Peltier and cools the active side. This activation layer is then attached to the back of the iPhone. 

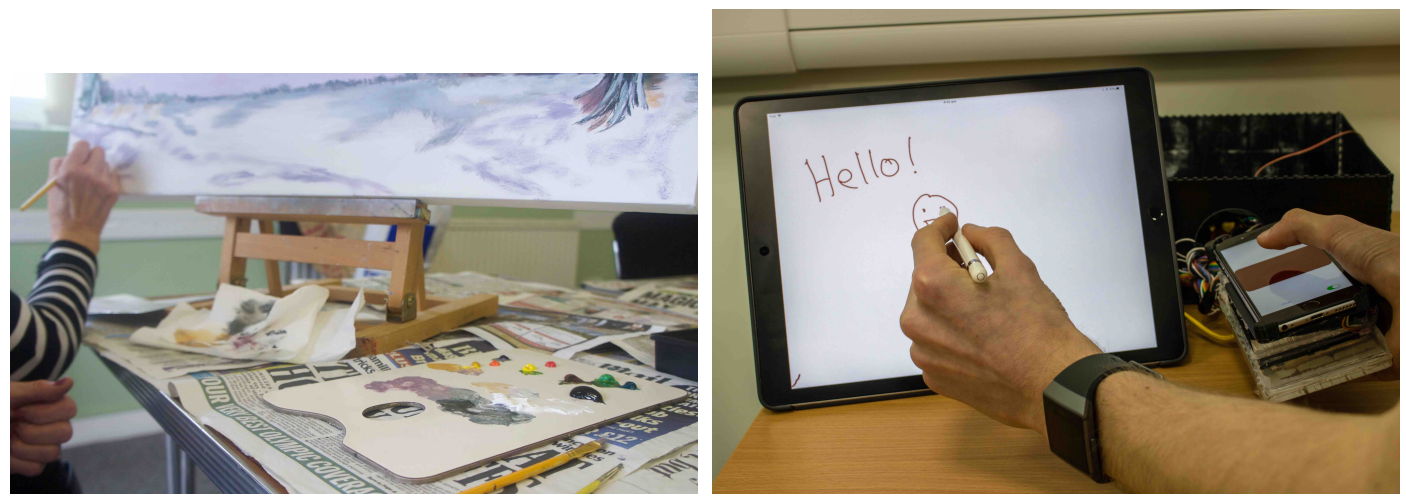

Figure 4.10: Left: Artist using palette and canvas. Right : Our digital prototype with tablet canvas application and mobile paint palette.

As all interactions from the back of the device needed to translate to how the paint is displayed on the front screen, we developed a paint mixing app. This iPhone app connects via Bluetooth to the prototype attachment. This allows the app to translate the coordinates of the interactions with the gel transmitted and display paint on the front screen. The app starts by displaying a set of primary colours (red, yellow and blue). From here, users can then drag these colours to other areas of the screen and mix them with other colours, all using the back of the device. Where the colours are dragged, these areas will also actuate to soft when the Peltier cools down the hydrogel where the paint runs from point to point. The application pairs with an iPad app that presents a canvas. Users pick the colour on the iPhone app with a stylus or finger, which they use to paint on the canvas. Our iPad app offers basic tools such as nib size adjustment and an erasure.

\subsection{Artist's Impressions of the Prototype}

We returned to the art class where we originally studied traditional painting techniques in order to demonstrate the prototype and give the artists an opportunity to use it. The feedback session took place over the course of two art classes with five 


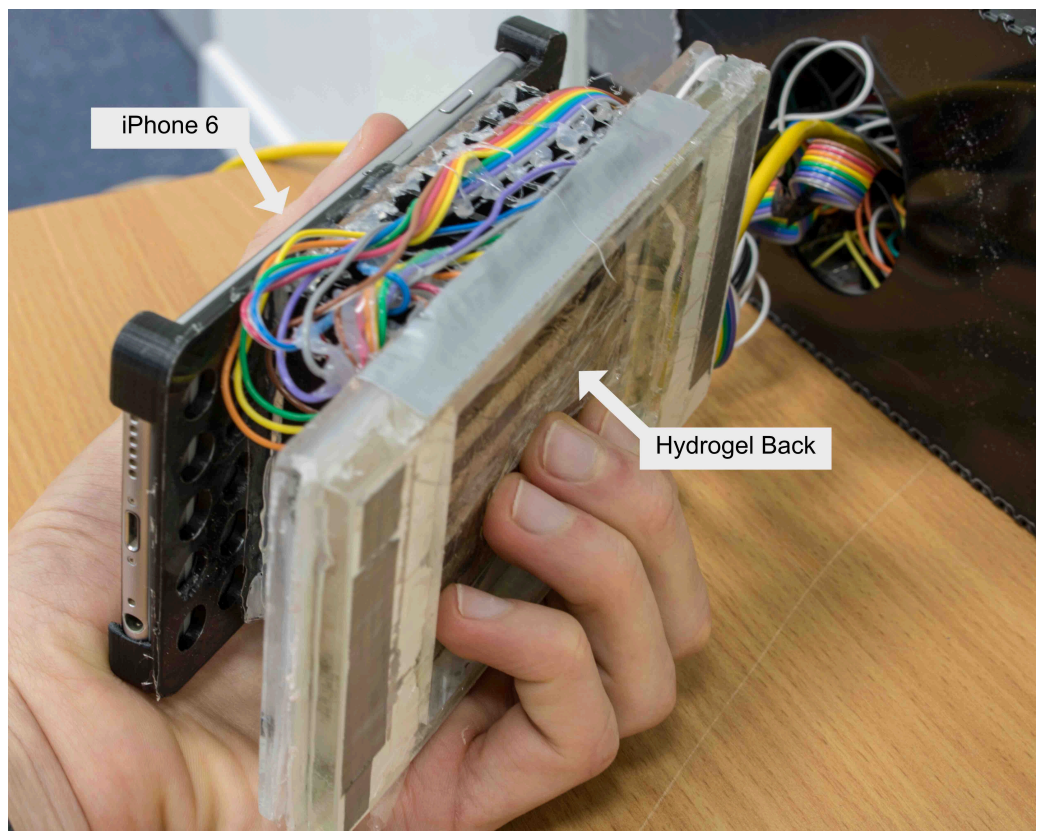

Figure 4.11: This figure shows the prototype as a whole from a side view, showing both the back gel screen and the mobile phone.

artists, and during the tea/coffee break. The artist's ages ranged from 46 to 72 and all had been practising their art for over 10 years.

We demonstrated the system's functionality and outlined its goals, and the artists then used the system individually. During this time, we showed them how to pick a colour and then told them to feel the back of the screen to mix it. We asked them to talk aloud about how it felt and compared to real paint. During this time, we observed and recorded what they did. After this, we spoke to them as a group to gather further feedback, taking notes throughout. These sessions allowed us to gather feedback about how the prototype compares to traditional painting. Specifically, we wanted to find out if the material felt like paint to people familiar with paint's consistency. We also wanted to understand how the gestures mapped to their analogue counterparts. Finally, we asked if the artists had any suggestions for improvements or future features that would be of benefit to them or other artists. 


\subsubsection{Findings}

Each of the individuals who used the system was impressed that the gel felt like certain paints. Some particularly liked how the initial 'blob' of paint they laid on their real palette was like how our system starts with the gel in the thick state. They explained how its behaviour was similar to flattening and spreading out the paint blob to mix their colours. As a group, they agreed that the hydrogel was similar to the oils and acrylics, but that water paint was not as similar. They explained that the gel was still too viscous, and they could notice that it was different from water paint even when it was fully cooled to its softest state. One participant explained that the softest state was more like a watered down version of acrylic paint.

For two of the artists, we observed that the thick blob that appears at the start of the interaction helped them detect where the finger needed to be to mix the paint. The rest of the group would simply glance at the back once to position their finger. One of the artist's main feedback points was about the use of knives with paints, explaining that this was her preferred method of painting. She suggested how adding this use case to the gel would appeal to her. This prompted another artist to suggest brushes too. This feedback gives direction to consider how tools could be used with the gel in future work.

Another area of feedback was about the device's limited output. The artists explained how the back felt real and interactive, but what was seen on the front was still only a flat digital colour. One artist asked if it would be possible to scoop up the gel to spread on the canvas, as tapping the colour after mixing is not as exciting as scooping real paint.

All the artists explained how the prototype was interesting but that it was unlikely to make them consider switching to a digital tool in any serious manner. Some explained that they had only just learnt to use real paint effectively and this would require more learning. Another person explained how they would not have 
the technical knowledge to set it up and use it. One explained how they would like to play with it a little more but said how they would be more likely to use it with their children for fun.

Overall, the traditional artists gave us valuable feedback. From these insights, we learnt how our device did have resemblances to oil and acrylic paint, but was still lacking the sensation of the softest of water paints. We also saw how the hard blobs of hydrogel on the back of the prototype simulated another element of the physical painting experience, and how it helped some artists find the intersection points to interact with. Finally, despite the artists not wanting to adopt digital art into their main workflow, they enjoyed using the system and thought it had value in effectively imitating oil and acrylic paints.

\subsection{Evaluating the Gel States}

After collecting the feedback from the artists, we wanted to further explore how similar the acrylic and oil paints felt relative to the gel stimulus. To find this out, we conducted a lab-based study using the gel and prototype. Our aim in doing this was to evaluate to what extent the acrylic and oil-based paint are similarly perceived to the hydrogel in a more quantified method. To measure this similarity, we conducted a task with paints and hydrogel, which were presented to participants under a cover. Their main objective was to determine which was oil paint and which was acrylic paint, while some were switched with hydrogel in different states of activation. We also used this opportunity to show participants the prototype and gather further feedback on their experiences. This section fully documents the study and the results obtained. 


\subsubsection{Method}

The user study was conducted with 16 participants. The first part of the study aimed to understand to what extent the soft actuated material hydrogel compared to traditional painting materials. Then, in the second part, we gathered feedback on our new mobile digital painting prototype. Each study took on average 20 minutes each. Users were compensated with a $£ 5$ gift voucher.

The study consisted of two tasks. In the first task, the participants felt hidden liquid materials, labelled them and rated the confidence of their label. There were three different materials for the participants to interact with. These consisted of hydrogel, oil paint and acrylic paint. The hydrogel was presented in two states, hard and soft and provided a total of four pots for the participant to feel. Each of the pots were covered by a plastic film to prevent direct contact. Then the pots were hidden from view and the order was randomised, and participants were not told about the hydrogel pots.

One by one, the pots were placed under the cover and the participant asked to touch the material in the pot. They then labelled it and rated their confidence of the label on a scale of 1-7. Participants were not told that two of the pots contained hydrogel, and therefore only labelled the pots as acrylic and oil. During the touching, we encouraged the participants to talk aloud about what they were touching and how it felt. Participants were asked to perform the matches as quickly and as accurately as possible, but we did not impose a time limit.

The set-up involved the participants beginning by sitting at a desk with a small table-like object with a cloth coming off the sides on the desk. We placed each of the pots inside the cover for the participant to reach under the fabric for the task. This meant that the material was covered from the participants' view without them needing a blind fold. 


\begin{tabular}{lccc}
\hline & Physical Paints & Desktop Paint & Touch Screen Paint \\
\hline Daily & 0 & 2 & 2 \\
Weekly & 2 & 3 & 1 \\
Monthly & 2 & 6 & 4 \\
Yearly & 3 & 3 & 3 \\
Less & 9 & 2 & 5 \\
\hline
\end{tabular}

Table 4.1: Frequency with which the participants used different painting tools

In the second task, each participant was given a chance to use the hydrogel prototype to mix a colour. During their interactions, we observed and recorded how they used the prototype and any immediate opinions shared. After this, we asked further questions and opened a discussion about the device's features, plus potential improvements and future use. The set-up for this involved the participants beginning by sitting at a desk with the prototype directly in front of them ready for use.

\section{Procedure}

At the beginning of the study, we greeted participants and introduced the task. We then competed the ethics procedure and demographics collection. We then started the first task. We placed the pot under cover and recorded what they guessed it was and their rating of how confident they were in their guess. We repeated this for all of the pots according to pre-defined latin-square order. We then started the next task where the users were given the prototype to mix a colour. We asked them questions about their experience using the prototype. 


10 daily weekly monthly yearly $\square$ less

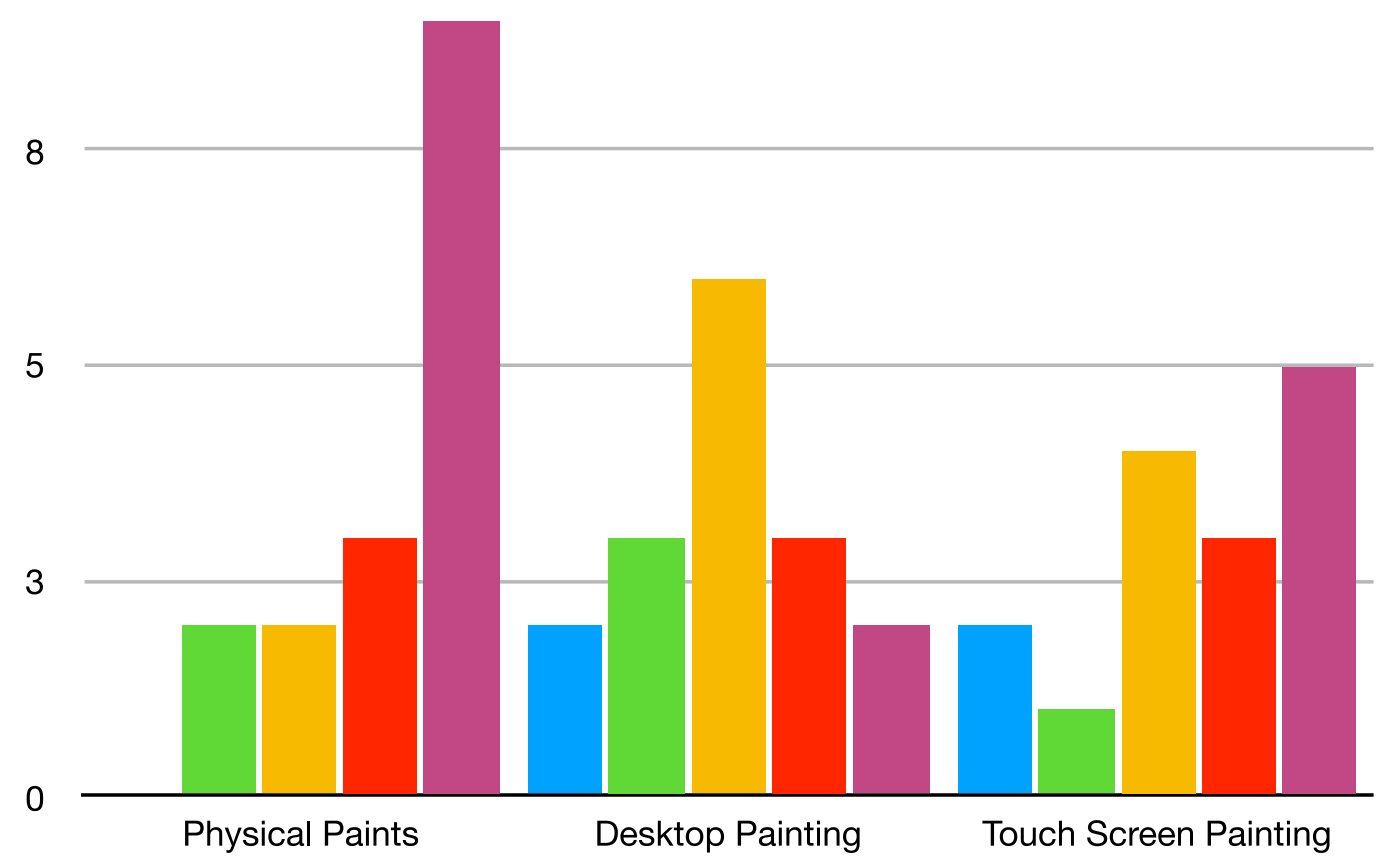

Figure 4.12: Number of participants that used different painting tools at daily, weekly, monthly, yearly and less frequency

\section{Measures}

Before the task, we collected demographic information. During the task, we recorded the user's labelling of the materials and their confidence rating. During their interactions with the prototype, we recorded their interactions and audio recorded answers to questions. We also recorded audio and encouraged the participants to talk aloud about what they were feeling.

\subsubsection{Results}

\section{Demographics}

The lab-based user study was conducted with 16 participants, six of whom were female and ten male. All were within the age range of 19-26. One participant was 


\begin{tabular}{lcc}
\hline & Oil Label (\%) & Acrylic Label (\%) \\
\hline Oil & 92.2 & 7.8 \\
Acrylic & 4.7 & 95.3 \\
HG Soft & 37.5 & 62.5 \\
HG Hard & 89.0 & 11.0 \\
\hline
\end{tabular}

Table 4.2: Percentage of participants who labelled each material as either oil or acrylic

left-handed, and overall the group had an average of eight years' experience of using touchscreens. Of the participants, nine had experience with using oil paints, 11 with water paints and 12 with acrylic paints. Table 4.12 below shows the frequency with which the participants used different painting tools.

\section{Identifying Liquids}

The total number of correct guesses are represented in a graph, which is shown in Figure 4.13. Table 4.2 shows the percentages for the participants who correctly labelled each material. We used a t-test to compare the accuracy of the participants' labelling of each material. We saw a statistically significant effect in the case of participants selecting the oil correctly as oil over the soft hydrogel as acrylic $(t=-3.98, p=0.00, p<0.05)$. Participants were also significantly better at labelling acrylic paint correctly as acrylic rather than labelling both soft hydrogel as acrylic $(t=-3.95, p=0.00 p<0.05)$ and hard hydrogel as oil $(t=-0.96, p=0.35, p<0.5)$ correctly. The participants were more accurate when choosing the hard hydrogel as oil than the soft hydrogel as acrylic $(t=3.23, p=0.00, p<0.05)$.

\section{Identifying Liquids Ratings}

Table 4.3 shows the results of the participants' confidence rating and the standard deviation of Task 1. For our analysis of the confidence rating, we used a Friedman 


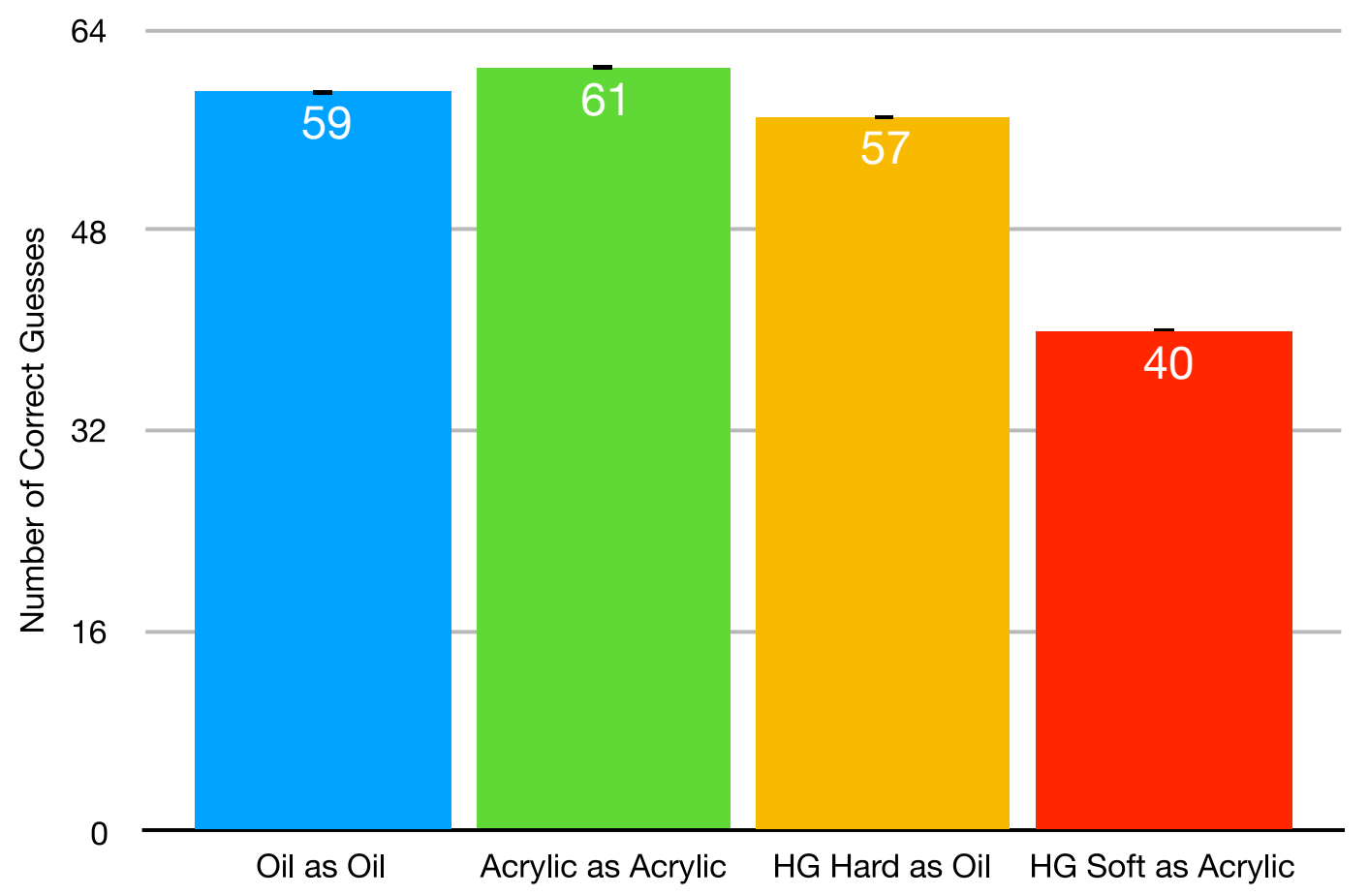

Figure 4.13: Number of correct guesses for each paint, including the error bars.

test to see if there was an overall effect. We then used a post-hoc Wilcoxon signed rank test to evaluate individual differences. There was a significant overall effect $(z=19.77, p=0.00)$ on the confidence rating depending on the material. The posthoc analysis showed that participants were more confident picking acrylic rather than acrylic over oil as oil $(z=262.0, p=0.04)$, oil as oil over soft hydrogel as acrylic $(z=339.5, p=0.02)$ and acrylic as acrylic over hydrogel hard as oil $(z=199.0, p=0.00)$.

\section{Using the Prototype}

While the participants used the prototype, we asked questions and observed their interactions. We transcribed all the interviews and analysed the scripts using a thematic analysis to identify common themes from the questions we asked. Firstly, we asked them to describe what they were doing on the back of the screen when mixing the colour using the gel. They described using gestures associated with 


\begin{tabular}{lcccccc}
\hline Confidence & Overall & SD & Correct & SD & Incorrect & SD \\
\hline Oil & 5 & 1.1 & 5 & 1.1 & 4 & 0.6 \\
Acrylic & 6 & 1.1 & 6 & 1.2 & 4 & 0.8 \\
HG Soft & 5 & 1.3 & 5 & 1.3 & 3 & 1.4 \\
HG Hard & 5 & 1.6 & 5 & 1.4 & 5 & 1.2 \\
\hline
\end{tabular}

Table 4.3: Median confidence rating participants labelling in task 1. We show the Median confidence of all the labelling together in under overall. Then the confidence of all correct labelling and incorrect labelling. SD = Standard deviation

paint. Some participants would rub the back, moving their finger round in small circles. P15 described this as "like rubbing in the paint on a palette" and P10 like "massaging the gel". Another interaction commonly mentioned was squeezing, and here we saw participants pushing their fingers into the gel, but little in terms of movement. The other key interaction seen was dragging. P2 explained how she was dragging her finger up and down to change the colour. A unique case was where one participant described that they were tapping the back to change colours.

Secondly, we asked the participants about the feel of the material on the back of what they were interacting with. Twelve of the sixteen participants compared the material directly to an oil paint. Of the twelve participants, four went on to explain that it was slightly stiffer, and P2 explained that it was "kind of like oil paints but holds its shape better". Only one of the participants compared it to acrylic paint. Participants also used other comparisons. P1 said that "it's like chicken breast under plastic", and P5 compared it to a rubber phone case, while P9 stated that: "it feels weird not like anything I've felt before". We also asked about their perception of the change in viscosity during actuation. We observed that most of the participants were not able to detect the viscosity change as the paint on the screen dropped in saturation. Only six participants mentioned any feeling of change. P10 explained that "it was goopy - now its quite flat - it defiantly feels like it's loosening up." Meanwhile, $\mathrm{P} 2$ described how the change was mapped to their interaction, saying "it seems to go down the same rate as I stir it". 
The main feedback we received for suggested improvements related to the difficulty of knowing where the finger is when hidden behind the phone. Four participants individually suggested adding a cursor graphic to the screen so that the position would map to the finger touching the back. P6 wanted to add pressure indication alongside the cursor.

We also received a lot of positive feedback relating to the user experience. All the participants enjoyed using the prototype and stated it to be a fun and unique experience. One participant explained that "it feels more 'hands on' compared to interacting with a screen", while another said, "you can literally feel what you're doing on the screen", referring to the paint changing. P3, an experienced painter them-self, explained that "it's like having a palette on the back of the screen," then went on to suggest that it was "good for people coming from a traditional painting background".

Finally, P1 summarised their feelings as "it always annoyed me in Photoshop ... you have to pinpoint a selection on a palette and then you have to play around will the sliders ... [I] often just find a colour online and use the Hex value, ... but you don't really learn how colours flow into each other, whereas this shows colours changing gradually".

\subsubsection{Discussions and Limitations}

An argument could be made for the hydrogel providing a good simulation of mixing oil paints. We saw no significant difference in the accuracy of labelling the hard gel over the oil paint. Neither was there a significant difference in the confidence of the labelling. This is also further backed up in the interviews where many directly compared the gel to oils, even after changing the states.

We saw from the conversations with the participants that the actuation of the gel did not seem to change the feeling of the paint. Some of the participants reported 
how it felt a little softer but did not change their opinion of it feeling more like an oil paint. This suggests that the actuation only provides a softening of paints as it is thinned out. Therefore, we are not able to accurately actuate between the two different paint types. When we compared this finding to the data from task 1 , we saw that $37.5 \%$ of the labelling for soft gel were marked as oil, a significant difference when compared to the labelling of hard state gel. This suggests that users are more likely to perceive the gel as oil paint. To further suggest this, the participants were significantly more accurate and confident when labelling the acrylic paint over the oil paints. From this, we concluded that acrylics may have an easier to detect viscosity, a viscosity that was less likely to be felt in the gel.

Its worth surfacing a potential contextual bias that may have existed during the study related the participants expectations. As when switching between the gel's sates and the two paint types the participants where always expecting paint due to the labelling. So, when touching the gel even if it felt different, they were still forced to think in terms of paint even though they though it to be completely different. We attempted to mitigate this with the confidence rating, so in such a scenario the participant could give the lower rating. Furthermore, the confidence rating and the matching accuracy contributed to the overall test of how similar the materials were in viscosity to crate the realism of paint seen in the contextual entry.

Another point of interest was how well the prototype afforded physical paint mixing gestures. We saw participants stirring and rubbing the gel as you would on a paint palette. Some of the participants even tried using dragging gestures, another common way to mix paint on a palette. When we compared the results from the lab-based study to the study conducted with the artist, both reported that the gel felt like mixing oil paints. Where we saw a difference was when the artists also thought that the soft gel was like a watered down acrylic, whereas, the labbased study participants either did not notice it or felt that it was still like oil after actuation. Overall, like in the case of the artist study, the participants enjoyed the 
experience of using the prototype. They liked the hands-on elements and texture feelings. Highlighting it was a more engaging way to interact with digital paints.

This use of programmable liquid tangible controls holds potential for bringing different forms of tangible interaction with digital art. Our prototype has shown early potential with existing hardware and accessible chemistry. Our future aim for the prototype is to deploy it with artists over a period of time. This will allow us to gather expert insights as to whether the device is valuable to artists. Alongside this, we also wish to improve interactions with the gel. We saw the key role different tools had during our observations, but currently the gel only works effectively with the user's fingers due to activating the touch sensor behind it. The inclusion of tools such as brushes and knives holds potential for our eventual vision.

Hydrogel, so far, has worked well in achieving our goal of tangible sensations of paint for digital painting experiences. During the development and evaluation of the prototype, we encountered several limitations of using the gel for mobile art-based technology. While we were able to create paint sensations for the artists, the development of a mobile hydrogel palette for everyday use may raise a range of physical and technical challenges. For example, the temperature of the area in which the gel is stored impacts its longevity. Keeping the gel in conditions at room temperature and above can cause it to dry out. In this case, the gel would harden, but would not be reusable when cooled. This evidently imposes limits on where such an approach can be used, perhaps even excluding users in extreme climates.

In order to maintain the gel's functionality, it needs to be kept fresh and uncontaminated. We achieved this by encasing the gel in a clear plastic film. While this removes the issue of contamination, the film limits the interaction the user can have with the gel compared to real paint. Users cannot, as one artist requested, scoop the gel out to place it elsewhere. Currently, our prototype is limited to dragging, pressing and rubbing on the activated surface. Scooping and placing the gel would be a further challenge in making our system closer to the traditional paint experience. 
The gel was not able to effectively simulate water paint for the artists. This is a limitation of the gel itself, as it needs to be of a stiff enough base consistency to simulate oil and acrylic paints. To make the gel more water-like will require the addition of more liquid. This will make the actuation slower (or cause it to fail to stiffen), affecting the user's experience with the oil and acrylic simulations. It will also require more heat, something the system currently cannot provide.

The final limitation of the prototype is its opacity. Artists were originally confused about why the gel was on the back of the screen and why the colours were on the front. We see this interaction as a way to provide an additional tool that users can hold in their non-dominant hand and interact with as they hold it naturally. However, being able to colour the gel itself and transport it from palette to canvas might provide a more seamless interaction. Overcoming or mitigating these limitations in future work would help in bringing seamless tactile painting interaction to mobile technologies. The next stages will study it is potential for bringing more engaging forms of interaction with digital art for artists.

\subsection{Summary}

In this chapter, we introduced a method for providing tangible texture manipulations and simulating paint mixing. This was based on insights gathered from the role of tangibility in traditional painting and its relation to digital applications, based on observations and interviews with experienced artists. We highlighted the role of texture in mixing paint and how it's missing in digital art interfaces.

This finding, lead us to develop a prototype that begins to facilitate how this texture experiences for digital paint mixing. Using the hydrogel we showed how a back-of-device screen could change texture based on temperature. These changeproviding gestures such as stirring and mixing, paired with a colour palette application on the front facing screen so that users were able to feel the sensations of 
mixing paints on the back and view the visuals of paint mixing on the front screen.

The feedback gathered on the prototype, showed that it demonstrated the potential for providing the programmable liquid tangibility of oil and acrylic paint mixing. A further controlled study highlighted the gel's similarity to oil paints and how the prototype was a more engaging experience with digital paints and supported many of the gestures akin to the painters interactions with paint on palettes. In the next chapter, we extend our research into colour interaction, this time studying how deformable interfaces can support how designers interact with colour.

\subsection{Authors Contributions}

The concept and implementation behind this research was the authors. Support was provided by Swansea Chemistry Department to source materials and demonstrate how to safely prepare hydrogel. The author planned and ran the study sessions, analysed the results and wrote the paper with feedback from supervisors and other authors. Deepak Sahoo (paper co-author) is an expert on using new materials advised on the paper in HCI and Ian Mabbett (paper co-author) was the key contact in the Swansea Chemistry Department and helped produce the Hydrogel. 


\section{Chapter 5}

\section{Modular Colour-Swatch Screens}

The last chapter highlighted how physically involved the colour mixing processing is for artists and how the textures of paints were a vital element of these experiences. These findings supported and informed the building of an actuated texture interface capable of simulating these textures. In this chapter, we continue our research into using deformable interfaces by further exploring how we can support colour mixing. We now shift our focus to graphic designers, whose colour picking is unlikely to involve paint and can therefore give us a different type of interaction with colour to enrich with new deformable interfaces.

Our aim for this research was to continue our exploring deformable interfaces by further studying how we can support colour mixing. Our aim was to continue our focus on interaction with colour and explore this process with a different user group that colour also plays a large role in their craft, the graphic designer. For a graphic designer, the colour picking is unlikely to involve paint so, therefore, our goal was to work with groups of graphic designers, show them some possibilities deformable interfaces offer and build a prototype that captures their processes in a novel tool.

We ran workshops with designers to discover how they pick and use colours in a design. These workshops found a mix of digital and physical methods, each with their own benefits and constraints. We saw the importance of swatches which 
allowed for physical colour browsing comparison, like the palettes used by the painters. We also saw their use of innovative GUI-based tools which employ algorithms to generate colour schemes and provide inspiration for designers.

Given the modularity of swatches, we considered the use of modular interfaces where screens can be deformed to make new shapes that change their functions. Utilising this type of technology allowed us to build a system that had the physical benefits of the swatches seen in the workshop combined with the dynamic elements of digital colour discovery. Feedback on the final system highlighted how it provides a new expressive hands-on experience for designers, to make for a more playful and creative experience. Overall, the chapter contributes an understanding of simulating physical tools and extends the comprehension of modular screens and the role they play in the user experience they provide.

\subsection{Introduction}

As we have seen throughout this thesis, colour is an essential aspect of art and design. It is used to communicate information and emotion. This importance makes picking and choosing the right colours a critical part of any creative process. Colours have a purpose and are used in meaningful ways [9]. Designers know that greens are seen as natural, stable or prosperous, while reds signify passion, aggression or items of importance. So, whether it is for a brand's logo or technical data in a diagram, the colours that surround us require careful attention and design [67]. Picking and manipulating colours before the introduction of digital tools required mixing paints or inks and laying out colours in physical spaces with the use of swatches. This tangibility brought a level of engagement and enjoyment that is now missed by many artists and designers who now use digital colour tools. Yet, the digital tools offer their own set of benefits, such as ease of mass production, portability and precise colour picking. 
The work highlighted in this section paves the way towards thinking about colour mixing in a new innovative way. The research in [46] highlights that colour picking goes beyond sliders on a screen, while the work of Lucero et. al. [68] shows how highly thought out the design process is. Finally, we consider how the physicality of transitional colour interaction has influenced bringing tangible elements to digital colour tools. Overall, these works have claimed an enhanced user experience and parented novel interaction techniques. The research in [110] (see Chapter 4) gave an example of how we can specifically take elements from traditional art. Inspired by these works, we believe that it is essential to investigate how designers can benefit from tangible user interfaces that combine the benefits of both the physical and the digital world, a strategy used in exploration for other applications (e.g. Pierre Wellner's DigitalDesk [139]). To take this approach, we began by studying how we can design and develop tangible technologies via a co-design process with designers to enhance interactions with colour.

We have seen examples of tools that help designers have a more engaging tangible experience when interacting with colour $[101,58,110]$. Related to this we also see the work that studies the design process of designers to develop graphical tools $[46,68]$. Our work extends this by also exploring the process of designers to understand where we can specifically aid their colour picking. We do this uniquely by involving them in the design and explorations of tangible tools for the digital colour picking. We contribute an understanding of their workflow, the constraints they face and a unique tangible interface that begins to address them. This extends the knowledge of TUIs used in professional creative applications.

In this chapter, we set out to understand how tangible elements could be added to the designers' digital colour picking process and to create more engaging creative experiences for individuals and teams that combine elements of physical colour discovery and the digital manipulation. We engaged with two different design teams to understand their existing colour picking process, both analogue and digital. We 
discussed with them the benefits of both and identified how we could create a new system that would support existing processes and solve the problems designers face. This method produced a map of the critical elements to a designer's workflow with colour.

After this scoping, we implemented a system to address what we learnt from the designers. The outcome is DigiSwatch, a tangible colour swatch system concept that allows individuals or groups to discover and manipulate colour on modular screens. These screens can sense adjacent colours to provide complementary colour discovery, and each module has touch control for individual colour manipulation. Feedback from the designers shows that DigiSwatch is a playful and fun way to find colours stating how it would make them more creative and reduce the number of external tools needed. They also highlight its potential for collaborative interaction for groups of designers and not designers.

We document where the tools fit into their existing workflows and where potential improvements prevail. In this chapter, we summarise the co-design process with the designers, explain the design rationale behind DigiSwatch and describe its implementation. We then report on the feedback received from the designers and conclude by discussing the outcomes for HCI researchers and the future implications of tangible colour picking and the possible iterations of DigiSwatch.

\subsection{User Research Workshops}

In order to understand how designers use colour and the possible ways we could build new interactions to support their work, we collaborated with sets of practising designers from two different companies. One set was from a design agency and another was from a design department within a broadcasting corporation. We ran a 45-minute workshop session with each group and we focused on dissecting their workflow to become familiar with daily tasks. We then brainstormed around 
improvements to ease the process. Together we built a map of their design process (following the method outlined in the Sprint methodology [57]) and outlined how they might improve their processes. We then introduced the concept of TUIs to start identifying a solution. We gave them the tangible probes and interactive wireframes to help generate new ideas.

\subsubsection{Tangible Probes}

In the second part of the workshops, we gave the designers interactive wireframes and a small demo made on the tangible interface, Sifteo cubes [69] (see Figure 5.1). This demo was based on background reading and informal interviews with design students. There were used to support idea generation for tangible interface ideas that would support their workflows. We also aimed to gather feedback on the potential form factor and discussed what features would be needed. The form factor of the Sifteo cubes helped the group understand the vision to provide better feedback. Using the Sifteo cubes, we could quickly prototype simple colour exploration features in an existing interface. We built a demo for complementing gradient colours with a predefined set of hues. In the gradient demo, as cubes were added to the right side of the starting cube, they displayed lighter versions of the original colour. In the complimenting colours demo, adjacent cubes took on colours that complemented the colours of the cubes to which they were attached. The interactive wireframes were used to illustrate similar interaction scenarios but showed a greater range of colours (see Figure 5.2). Using these probes, we gave them a glimpse of how a future system could work and generate ideas.

\subsubsection{Creative Design Agency}

First, we visited the design agency. We conducted the session with a group of five people from the agency's design team (M:1 F:4). As a design agency, their work 


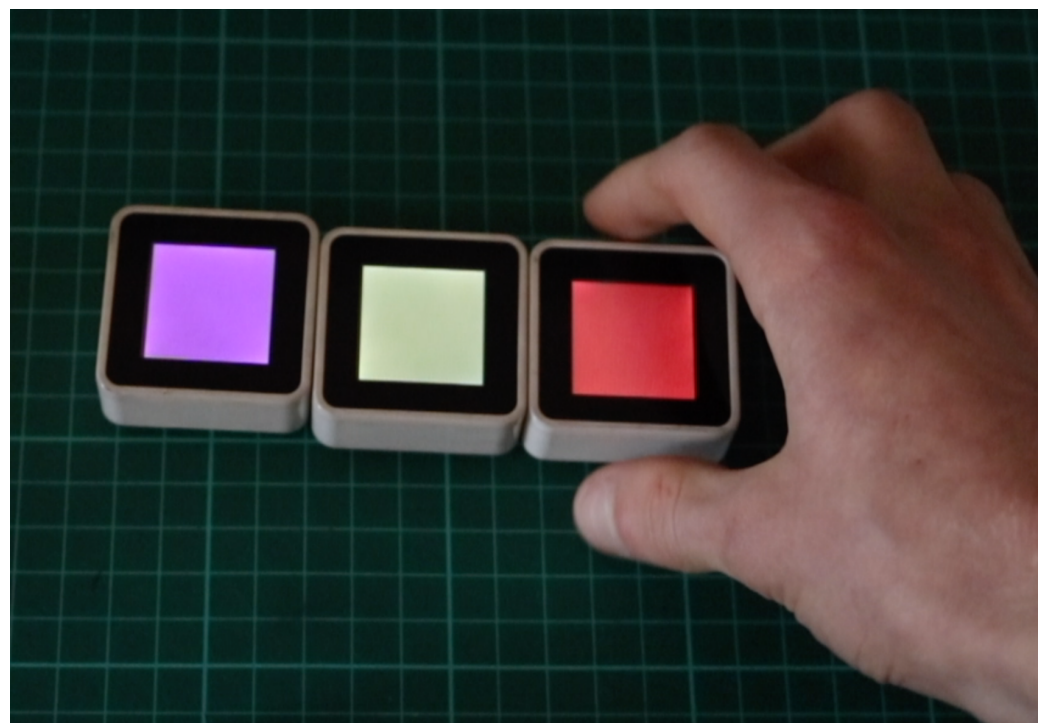

Figure 5.1: Siftio Cubes used in the workshops running the colour matching program.

predominately centres around clients who need branding and marketing resources. We opened our discussion by talking about how they go about picking colours when the client is branding from scratch, with no pre-defined brand guide. They explained that when they are collecting colours from scratch, they begin by looking at brands of a similar category. It aims to both inspire them and ensure that they are not copying an existing identity. Using this initial survey, they begin to build a mood board with lots of different colours and clippings of examples. They use websites like Pinterest ${ }^{1}$ to find the examples. They generate colours using tools like Adobe Colour $\mathrm{CC}^{2}$ or printing off colour swatches to view and compare.

Once they have crafted mood boards and decided on what colours they want to use, they present it to the client for feedback. They then iterate the process by implementing the feedback and present the new version to the client if required. According to the designers, the primary challenge at this stage is to explain the reasoning behind the colour to the client. Once signed off, they test the colours in different print formats and screen scenarios. These tests, help them build the

\footnotetext{
${ }^{1}$ Pinterest: https://www. pinterest.co.uk

${ }^{2}$ Adobe Color CC: https: //color , adobe. com/
} 


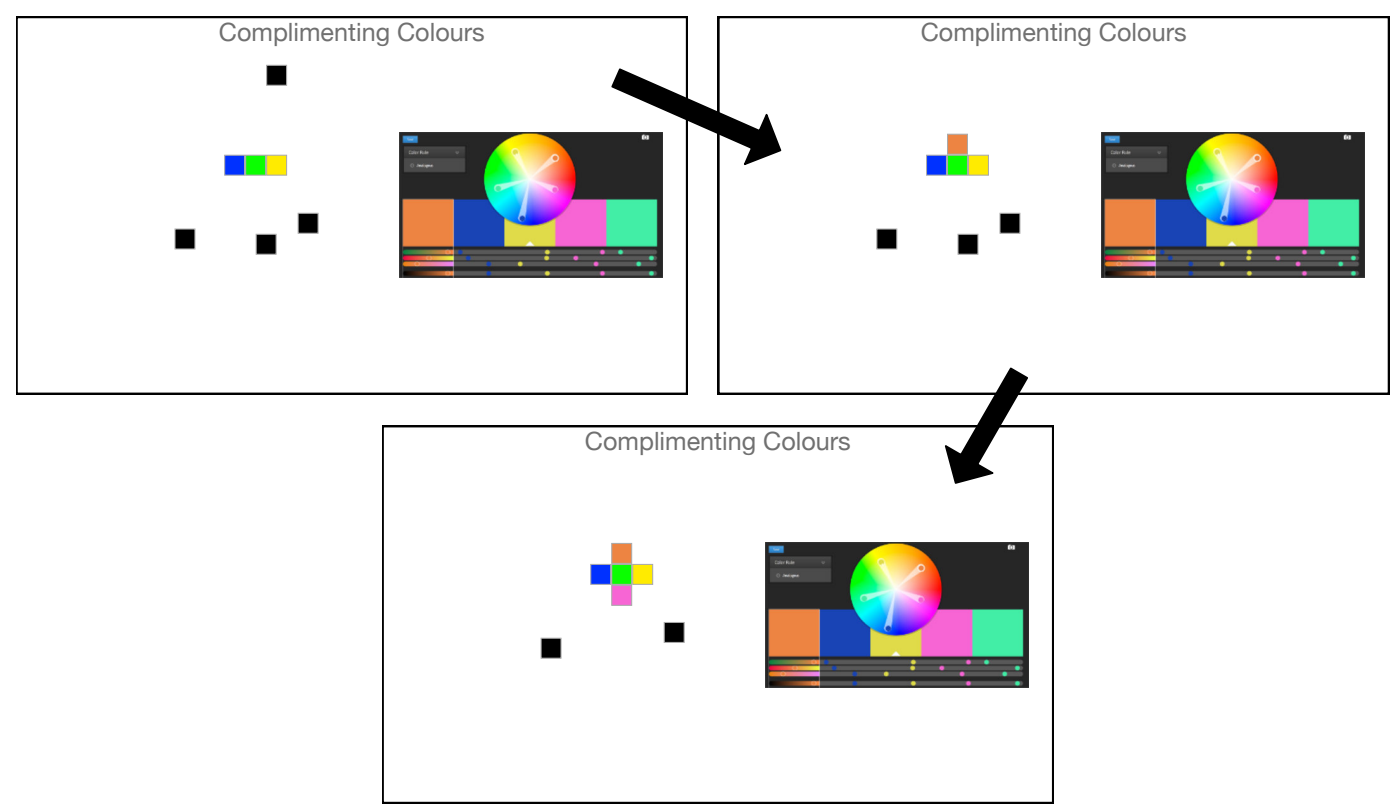

Figure 5.2: Images made for one of the interactive wireframes to compliment colours. Wireframes were made using Adobe XD

brand guideline documentation, which gives clients a set of rules on how to use the colour palette they have defined. The core idea of this guideline is to clearly state how everyone in the organisation has to use the colours without the need for the designer's supervision. The document provides hexadecimal codes for each colour and Pantone ${ }^{\circledR}$ reference numbers as well as the use case scenarios (e.g. official colour printed documents, flyers or posters). Once the guidelines are defined, they begin making the materials such as posters, leaflets or brochures. At this point, the designers may need to find some complementary colours.

Designers told us that a big challenge they face is communicating with clients. To help them, they use Pantone®bridge kits (see Figure 5.3). The kit they showed us had six different fans each showing the colours of different paper styles. The Pantone ${ }^{\circ}$ books are sometimes used in client meetings but are cumbersome in quick situations. If they are dealing with a new client, they will do all the colour picking in digital before looking at printed materials. We summarise the processes described above in the diagram shown in Figure 5.5. This figure outlines the main stages of 


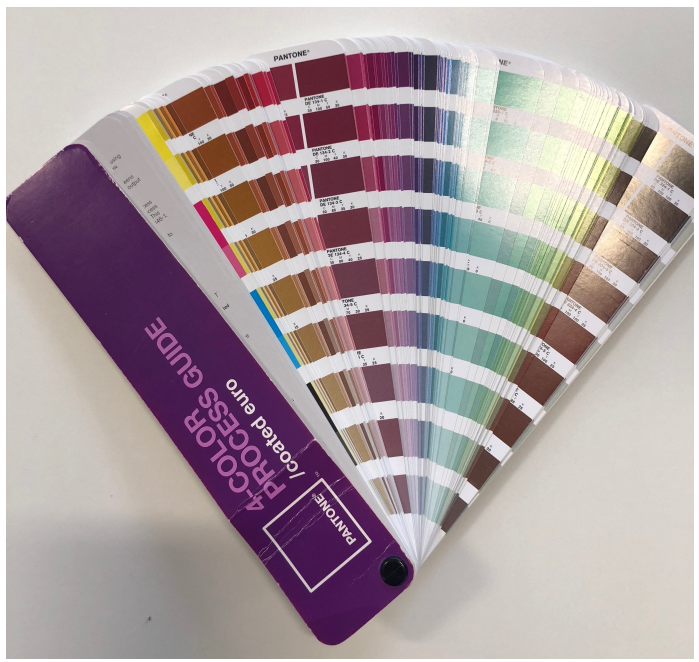

Figure 5.3: Pantone ${ }^{\circledR b}$ bridge kits used by designers with clients to determine colours for brands and resources. e.g. flyers, leaflets and posters.

the design process.

When using the Siftio Demo, designers liked the feature of finding complementing colours. There was also a high interest in the colour shade exportation which was displayed only with the wireframes, and not the Siftio cubes. They discussed how they would like to sample colours from an existing material the client would bring or from their mood board. When comparing it to the Pantone®books, they explained how they sometimes found that the books offer too many choices, which is overwhelming both for them and the client. The cubes give us just a couple of colours to focus on at a time. They also said that the books make it hard to compare the colours because a fan attaches them. The cubes are easy to place near each other for quick colour comparison. They added that Pantone ${ }^{\circledR}$ offers books with removable swatches, but that these were very expensive.

They saw potential in using it with clients and outlined how they could use these tools to show the client a swatch palette to get instant feedback. This feedback would save them time on going back and forth to adjust the colours. Finally, they added that the cubes would impress a client and get them excited in the initial 


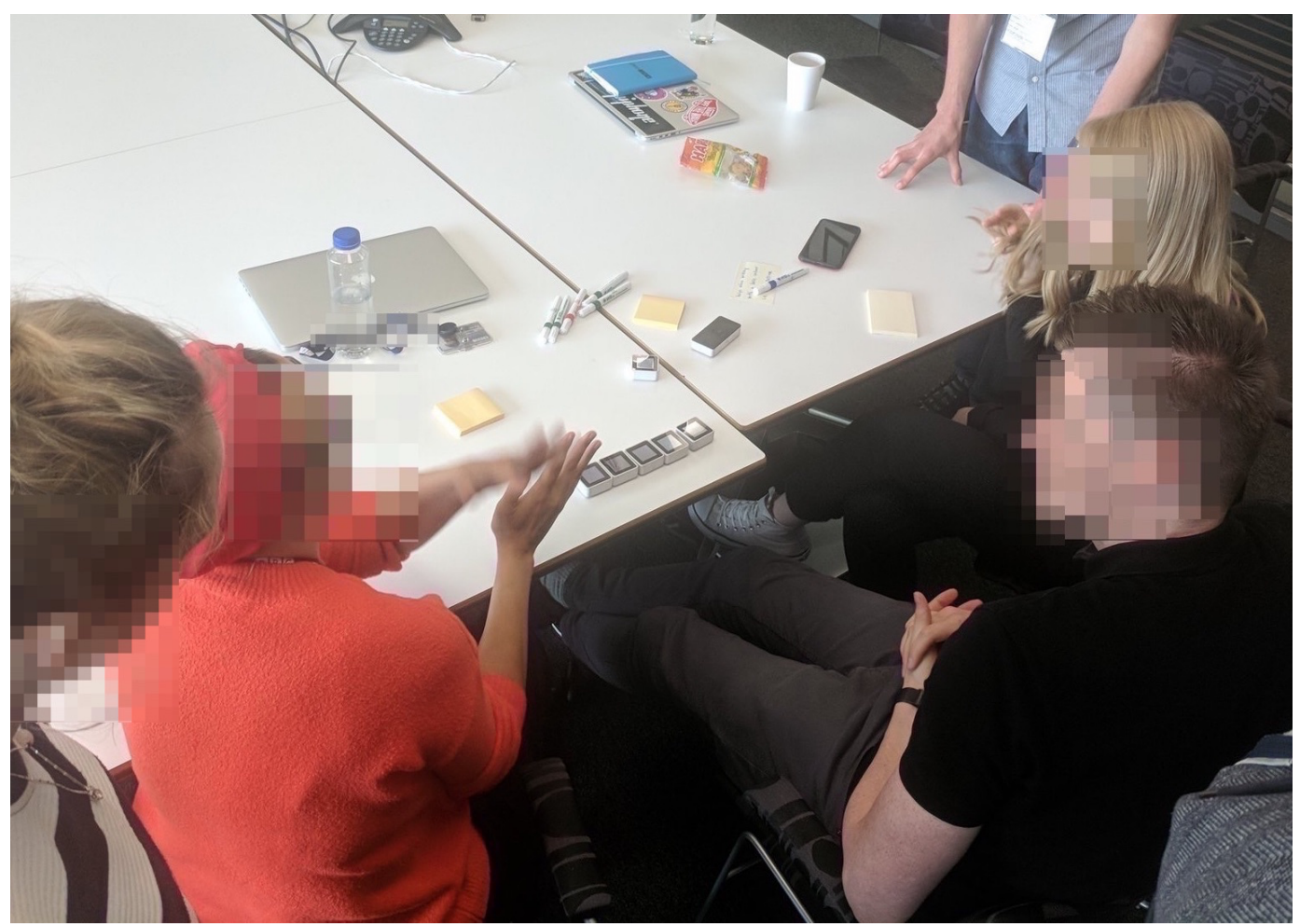

Figure 5.4: Workshop with the BBC UX\&D Designers

meeting. They thought that the tangibility would make the meeting more engaging for them to get involved in the colour selection. However, there were some concerns about screen colour calibration and the screen brightness. This screen issue could create inconstancy between the computer and prototype. They liked the ability to place cubes near each other for colour comparison, but they suggested minimising the bezel. One of the designers even suggested having the colours on all sides, like a dice. They would also like to see previous colours so that they can compare the change when looking for new ones. They also explained how they would like to select a starting colour from their computer. It would allow them to start with an accurate Pantone ${ }^{\circledR}$ reference colour from the client's brand guide. 


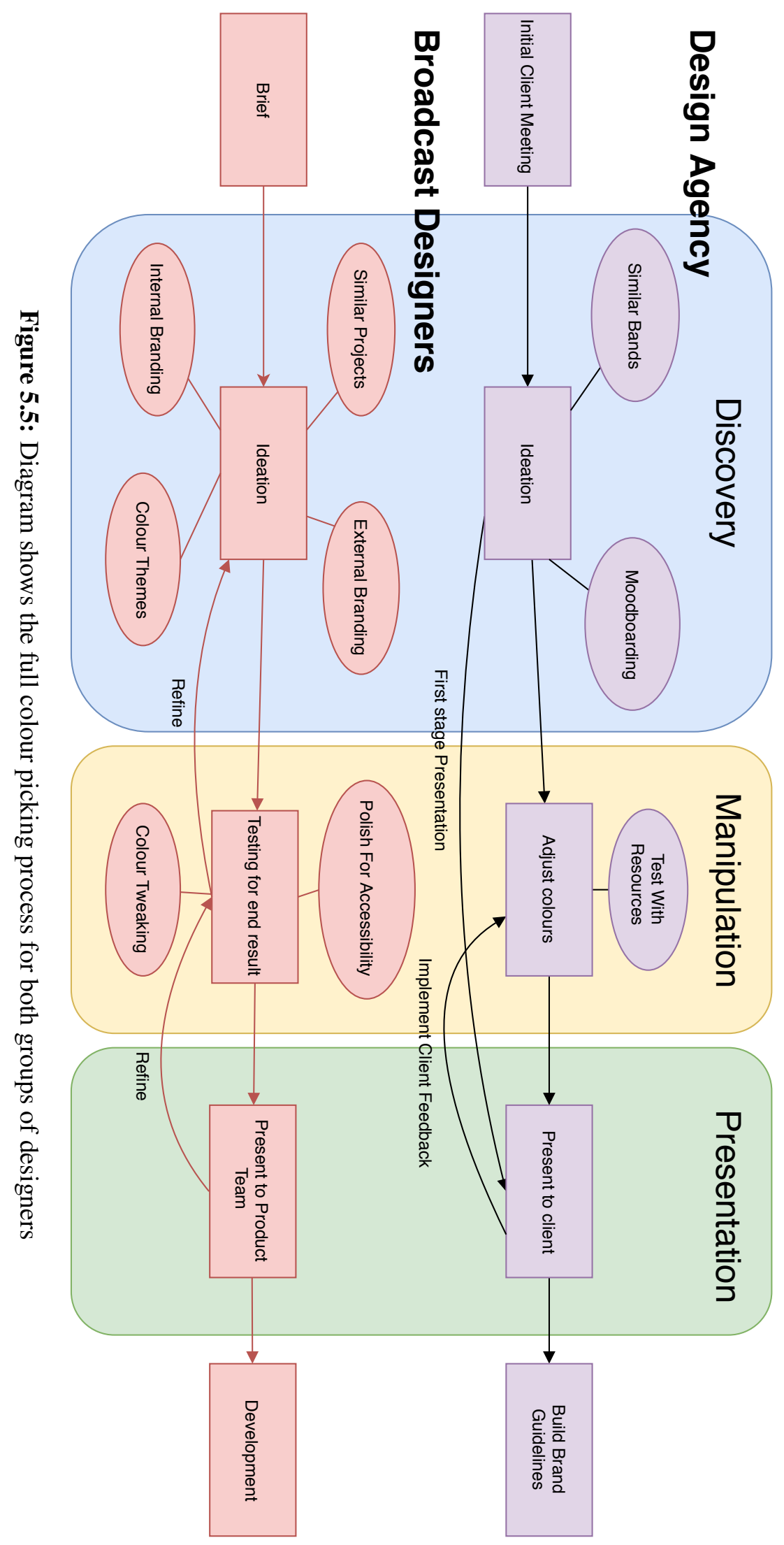




\subsubsection{Broadcaster Designers}

We conducted the second scoping study with designers with designers from BBC $\mathrm{UX} \& \mathrm{D}$, which was carried out with a group of five designers and one creative director (M:3 F:3). First we asked them about their current workflows. In the group discussion, designers reported that it was common for them to use an existing set of internal brand colours. Therefore, their job mainly consists of finding a set of colours that accompany the brand palette. Sometimes, they would need to incorporate an event's brand colours, e.g. The Football World Cup. For example, the BBC Sports needed to incorporate the World Cup colours into a section of their mobile application. In situations where they need to bring in new colours, the designers look at existing images and material with suitable colour schemes, then they tweak them as needed. They often end up only altering the shade and the saturation.

One of the designer's key roles involved data visualisation. She used colours to communicate data and was mindful of colour blindness. She explained that she is not a fan of existing tools for checking colour accessibility but uses them out of necessity, and this was unanimous with the other designers. The use of the Adobe Colour CC tool is common, but one of the designers expressed how they do not always like the colours it recommends, and therefore end up changing them a lot. Another designer disliked it, expressing how it is not always clear why those the colours appear and not others. One of the designers described that finding the right colour was mostly based on instinct. In her own words, she described it as "messing around until they look OK". Part of this process is making boxes of colour in software and then moving them around to compare. Another member of the team works in the area of content discovery which incorporates different products across the organisation. He and his team face the challenge of finding colours that work in an interface with lots of different product colours coming together. All of these have their set brands and rules for colour usage. We also discussed how the different design teams proceed to present and share their work. 
All designers work with the creative director to present colours. Their role is to challenge the colour decision and understand its effect. At this stage, they considered the physiology of colours. One example of these colour conversations given by a designer related to the colour red. An example discussion would be that red conveys is a warning, but is for indicating that a program has gone live. Other aspects could include using colours to highlight or focus users' attention. The designers often deal with noisy environments full of UI elements or videos, so using the right colours helps to limit users' confusion.

After introducing the Siftios to the designers, we observed lots of smiling and laughter. They engaged with the system and seemed to enjoy it, commenting with expressions such as "that's nice" or " that is clever, I want a red one now". When asked to comment they explained how they liked the ability to set the initial colour as most of the designers work in departments where the project has an initial starting colour. One articulated "I work in Sport, where you always need to start with yellow. With this, I will start with that then build around it". Another explained "it can be hard to pick a colour in the initial idea stage; I think this could help".

One thought it was more fun as it is gesture-based and thus more familiar. They stated that "it would be easier to swap colours around for different comparisons". Another designer explained how it would "potentially give an immense understanding of what colours go well together, like a discovery tool”. They also went on to explain how it would give a rewarding sense of finding colours. A different designer commented it would be like having a second opinion on which colour to use. The designer next to him added "plus it is playful", which is one of the broadcaster's objectives in order to increase creativity.

They also spoke about how accessibility is vital to them but usually gets tested in the end. They said that if this system had accessibility testing features built in, there would be potential to make decisions based on accessibility from the start. Features they suggested included: contrast ratio, colour blind glasses mode and highlighting 
parts of the gradient with the most suitable colours. The discussion then led to the creation of cube use case scenario based on the role the swatches should fulfil. For example, allowing the designer to decide if a cube's colour is a text or background. This would be suitable in order to test the contrast between colours.

The group raised several issues. They saw a limitation in the fact that the cubes were activated even though the designers did not directly use them. This factor was mainly due to a lack of sleep mode in the Sifteo cube, and we therefore chose not to turn them off. In order to be usable, the designers stated that they should be able to send their colours to the cubes, for instance, colours taken from a set brand. The fact that our study probe only allowed for a limited "hardcoded" set of colours was a concern. They also suggested the use of a second device to set and get the hex code of colour displayed on the cubes.

\subsection{Workshops Discussion}

Many similarities arose between the colour picking workflows of both groups. They both undertake some form of collecting and colour sampling from items such as photography or existing brand identities before they start manipulating colours. Out of the tools they use, the Adobe Colour CC, a tool for finding complimenting colours, is commonly used by the groups. In both groups, designers also need to present and articulate the rationale behind their colour theory to other designers.

However, only the agency group worked with external clients. This type of work is a crucial limitation as general clients are not colour experts, which refrains them from being engaged with the process. After seeing our example of a tangible tool they thought it would be a good way to for them to engage in the process as they can get the client to play with the colour and explain where the colour is coming from to help support their rationale. It seems that this point could be addressed 
with tangible tools since they saw in our prototype a way for clients to engage in the process by fiddling around with the colours.

Testing colours was another similar element of both processes. In the agency, they test colours in different scenarios, which helps them to have a ready-made brand guidelines document to give to the client. In the broadcasting group, the tests were more focused on accessibility, which involves making sure the contrasts of colours are appropriate for any visual impairments. Accessibility is an essential element for them. They therefore need system accessibility features built into integrating testing from the ground up.

The BBC group, and occasionally the agency group, have to build their design upon existing colour brands. This situation highlights the need for designers to be able to preload or send colours between colour pickers in order to use them in their designs. Finally, both share the same mixing and manipulating colour process when using a tool based on complementing rules. Both teams enjoyed using the Siftio Cubes, highlighting their potential uses for their colour picking.

We received good technical feedback recalling colour clarity, screen resolution and bezel size, which highlighted some of the limitations of using Sifteo cubes. Overall, feedback demonstrated that the use of tangible controls was a viable option. We therefore took into consideration all observations and feedback on our prototypes, to implement a system and map out how a such a system could be used.

\subsection{Design Framework}

After the visit, we mapped out the constraints of the designers' current colouring picking progress. Based on what we found we defined three main categories that define picking the colour. These are Discovering, Manipulating and Presenting and are llustrated in Figure 5.6. 


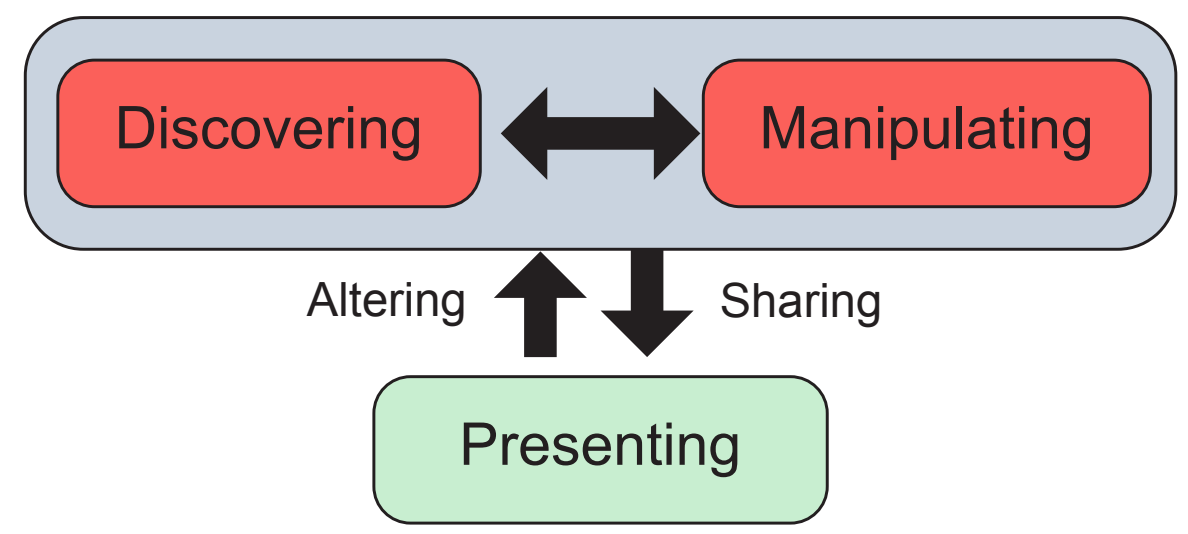

Figure 5.6: Overview of the steps a designer takes using existing tools based on findings

Discovering: Describes the process of looking for colours. The designers use tools like Pinterest to collect colours and organise mood boards. They also use tools like Adobe Colour to find complementing colours and sample different gradients.

Manipulating: Describes the point where the designers need to change a colour manually for individual purposes. This manipulation could be to change a specific colour channel such as hue, saturation or tone. It could be the mixing of colours, for example, they may want to make a red warmer, so they mix it with orange. The steps take place concurrently with discovery. When finding colours, they may not be accurate to the designer's vision. Therefore, they require manipulation.

Presenting: Describes the stage where designers come together to share the colours they have collected to give feedback. After this, they will return to discover new colours or manipulated the current colours. This stage also includes presenting to clients and stakeholders as we saw with the design agency. They will return to make the alterations and then present them again to obtain sign off. In the case of the broadcast designers, we saw a need for testing the 
colours' accessibility and understanding context to be of importance. This process takes place in a feedback loop of meetings with the product managers.

The top suggestion we received during our user research centred around making the colour picking more engaging and even more playful. Suggesting this element could support working with clients and managers to acquire more timely feedback and decisions. We consulted both maps and saw the steps when collecting the mood board materials, then presented the first set of colours, which was followed by adjusting the colours before then returning to the meeting to see if the adjusted colours could be signed off. In our diagram, the first box encapsulates the discovering and manipulating. The colours are shared in the presenting stage to obtain feedback. Then, based on the feedback, the designers return to discovery and manipulation.

We envisioned a system that allows the discovery and manipulation to happen seamlessly creating a more engaging process. This would combine the processes and enable group discovery and manipulation and make the presentation stage more engaging as designers and clients will have instant feedback (see the updated diagram in Figure 5.7). This engagement eliminates constraints that results from separating the presenting and looping back to alter colours. Overall we aim to address the needs of: easier to explain colours and contexts, to obtain client feedback and immediate decisions and to make colour picking more engaging for clients and establish complimenting palettes.

\subsection{DigiSwatch Development}

In order to achieve our goals, we set out to build a tangible interface that resembles the colour swatches seen in the workshop with the designers. These swatches would combine the benefits of tangible accessibility with the digital benefits of colour graphical colour discovery tools. This interface would make them dynamically 


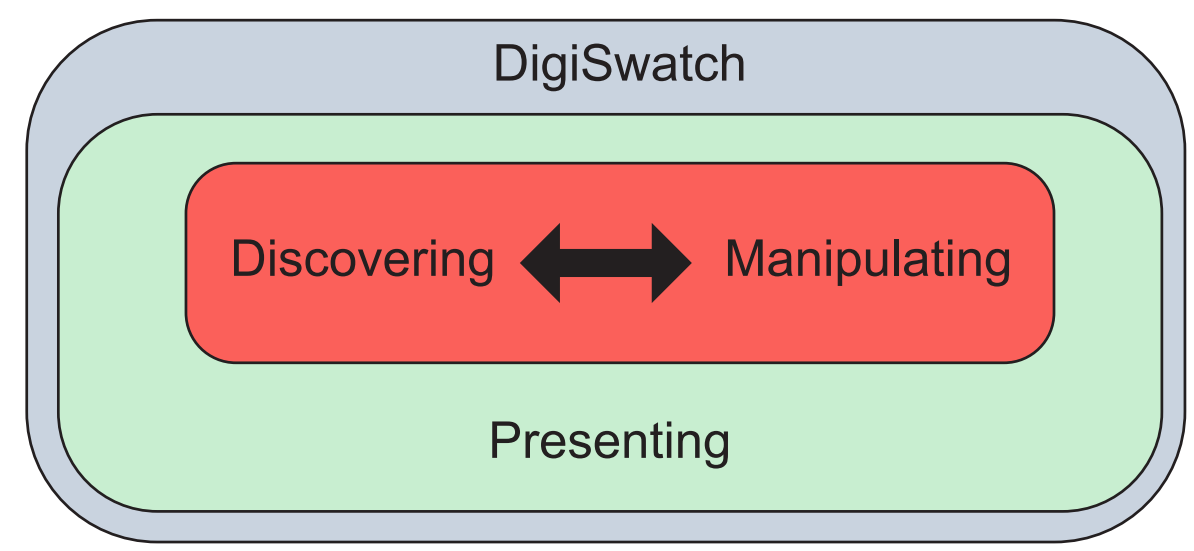

Figure 5.7: Our vision for how tangible tools can combine the elements for a more seamless colour picking process

changeable but movable in physical space. As the designers saw merit in the form factor of the tangible screens. We used this as an inspiration for the new prototype we built with added features implemented from the feedback received. We call this system DigiSwatch (see Figure 5.8).

\subsubsection{Hardware and Architecture}

DigiSwatch is a modular screen-based system which displays digital colours to designers. The system is composed of cubes ${ }^{3}$ Each cube integrates a smartwatch embedding a touch-sensitive display and running an Android OS which can run custom applications. The cubes are $3.5 \mathrm{~cm} \times 4 \mathrm{~cm} \times 2 \mathrm{~cm}$. Encased in each cube side are a lever switch and a magnet. The switch states get processed by a custom program running on an Arduino board placed inside the cube. The Arduino continuously streams the switches states to the watch via Bluetooth.

Each Android application communicates via Wi-Fi with a Nodejs server running on a laptop, whose purpose is to keep track of the current configuration of the cubes. Cubes can be physically connected using the magnets. When connected,

\footnotetext{
${ }^{3}$ Our current implementation contains six cubes. However, there is no theoretical limit to the number of cubes that can be used at the same time.
} 

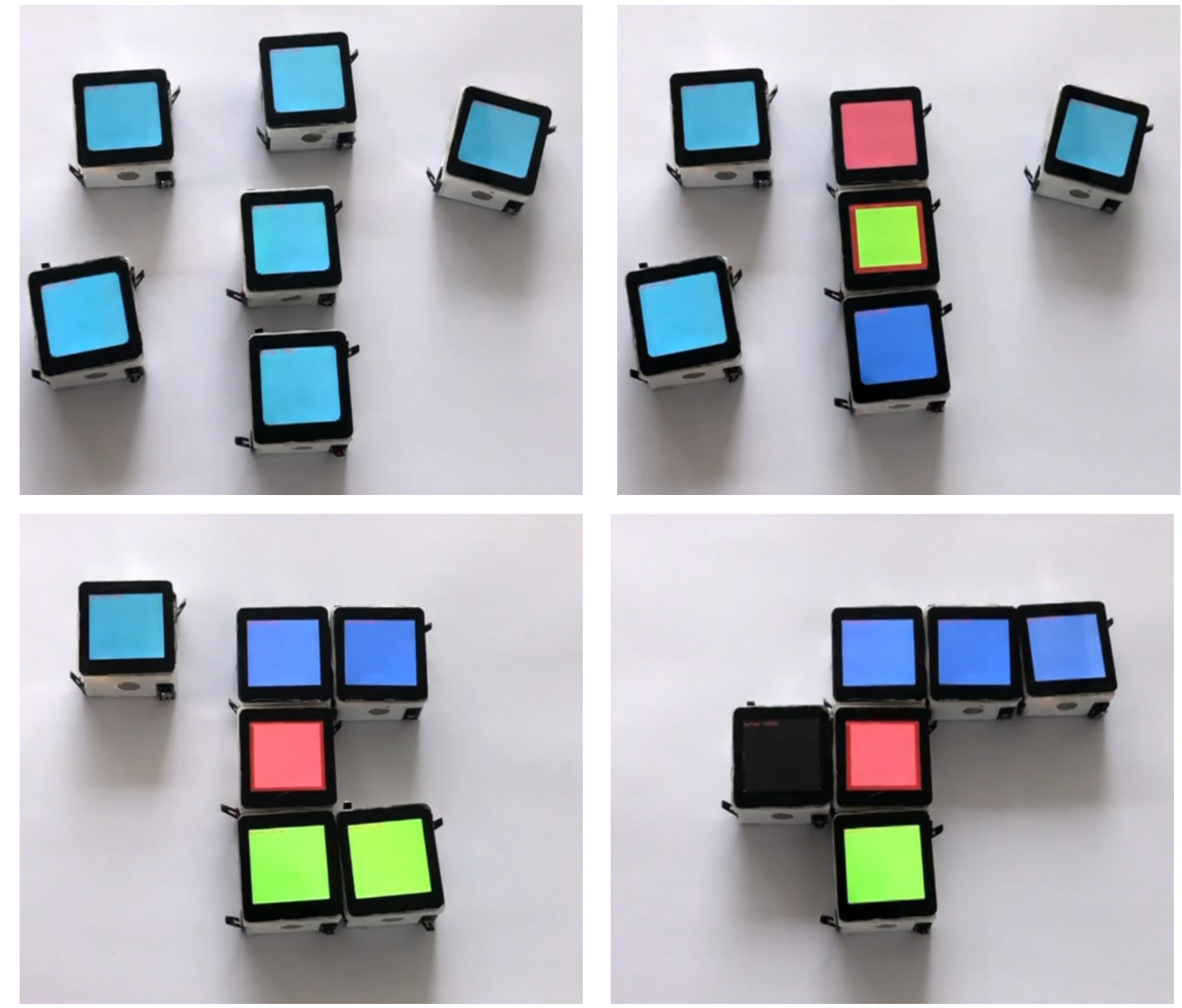

Figure 5.8: DigiSwatch : Figure illustrates how the combining modular screens deliver colour discovery and manipulation in groups of connected cubes

changes of the switch states indicate changes to the cubes' configuration to the server. Cubes also stream their touch events to the server. A C++ colour application running on the laptop interprets all the information gathered by the server and determines the different interactions and colours to display on the different cubes. Once determined, the application updates the display of each cube via the server.

Compared to the tangible probes used in the workshops we added some different hardware to resolve some feedback. We added magnets for a clear side connection and locking interaction, a paired desktop client to retrieve colours for use in design apps, and improved touch interactions and colour display using higher fidelity on 


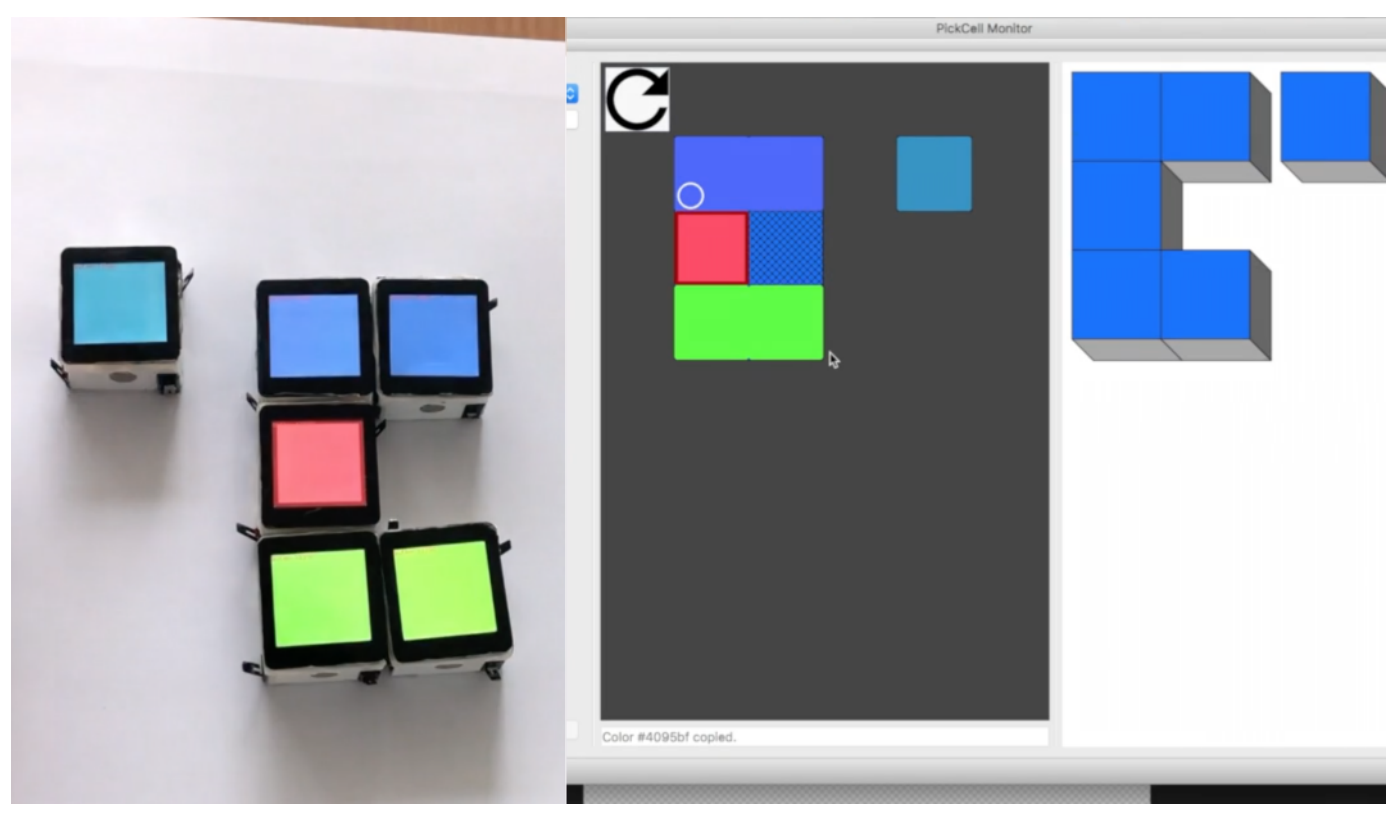

Figure 5.9: DigiSwatch (left) Software (right). The software shows the current configerations of the cubes. It also allows for sampling colours.

screen. For the purpose of our research, we linked the cubes to a laptop, but the cubes can be self-contained (e.g. self discovery when connected to the same Wi-Fi network) making them intrinsically mobile. Instead of our Node.js server, a webapp could be accessed from any laptop/phone/tablet connected to the cubes network to send/retrieve information from the cubes and use it in digital design software.

\subsubsection{Software and Interactions}

Our system enables colour exploration using a tangible colour swatch interface. Each cube represents a colour swatch. By default, a cube will update its colour in function of the current configuration and the colours explicitly set. All cubes are equal and any cube can be set to a default, master or contrast mode via long presses cycling through them (see figure 5.10a).

To set a colour on a cube, the user must change the default mode of the cube to the master mode by long pressing on it. Once the cubes are in master mode, 

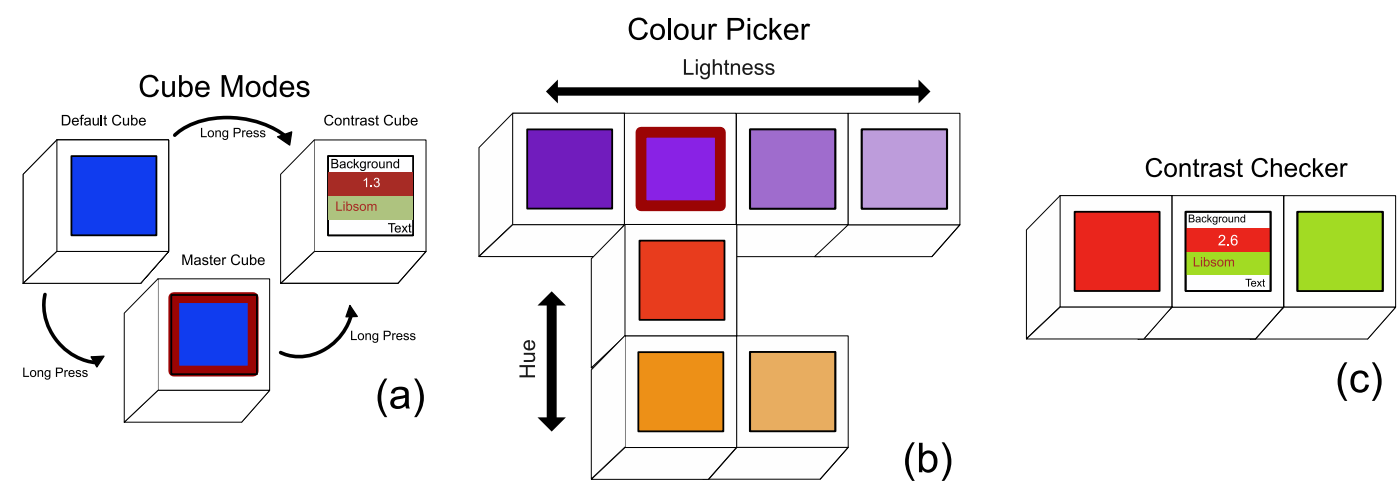

(c)

(b)

Figure 5.10: (a) Switching between different cube modes (default, master and contrast) via long press interactions. (b) Illustration of a colour picking layout. Adding cubes horizontally changes the lightness and vertically changes the hue. Each change is based off the master cube. (c) Contrast checker cube in the centre of two coloured cubes. Output is displayed based on both left and right adjacent cubes.

indicated by a red border (see figure 5.10a). Its colour can be modified using three sliders. Each represent a dimension of the HSL space: dragging leftwards on the upper part of the screen decreases the lightness/limerence value (L of HSL); dragging rightwards on the upper part of the screen increases the lightness/limerence value. Likewise, a horizontal slider on the bottom part of the screen changes the saturation value (S of HSL), and a vertical slider changes the hue value (H of HSL). We chose the sliders to modify the HSL space values as it was highlighted that these were the most commonly modified values by the designers.

Default mode cubes update their colour in function of the master cubes (see Figure 5.10b). Cubes on the left of a master cube will display the master cube colour with a decreased lightness value according to their relative position (i.e. the further away from the master cube, the darker the colour). Likewise, cubes on the right will display the master cube colour with an increased lightness value. If several master cubes are present, the default cubes in between will display a gradient between the set colours. Cubes above and below a master cube will display complementary colours. The displayed colours represent an equal division of the hue wheel starting from the master cube colour. The $\mathrm{S}$ and $\mathrm{L}$ values remain the same as the master cube 
colour. If several master cubes are present, the default cubes in between will display colours with an equal division of the portion of the hue wheel comprised between the master cubes. The $\mathrm{S}$ and $\mathrm{L}$ values of the cube are then linearly interpolated.

The colour of each cube updates starting from the master cubes. Every time a user manually changes a cubes colour or the cube's configuration changes, all the default cubes colour are updated by a single touch on a cube copy of the HEX colour code in the laptop clipboard. If the clipboard contains a HEX colour code (e.g. from Photoshop or another application), a control-click on the virtual representation of a cube sets its colour and automatically changes it to a master cube.

The final feature we implemented explicitly addresses colour accessibility (see Figure 5.10c and 5.11). An element extensively discussed with the broadcast designers was about their need to ensure that colours have the correct contrast for people with visual impairments. The colour contrast feature is activated by setting a cube to contrast mode, and this cube's output will then populate when adding colours on the adjacent left and right sides. From here, any cube added to the right is seen as the background colour and any added to the left is the foreground colour. The centre checker cube then takes each colour value and calculates the contrast. The contrast value is then displayed on the screen. Depending on whether the colour contrast is compliant with W3 accessibility guidelines ${ }^{4}$, we display the contrast value with a green background for compliant, orange for almost compliant, and red for not compliant. We also display a preview of the text with the foreground colour on the background colour as a preview for the designers. This tool allows designers to check colours for accessibility alongside the discovery features.

\footnotetext{
${ }^{4}$ W3 Accessibility Guidelines: https: / /WWW.w3 .org/TR/AERT/
} 


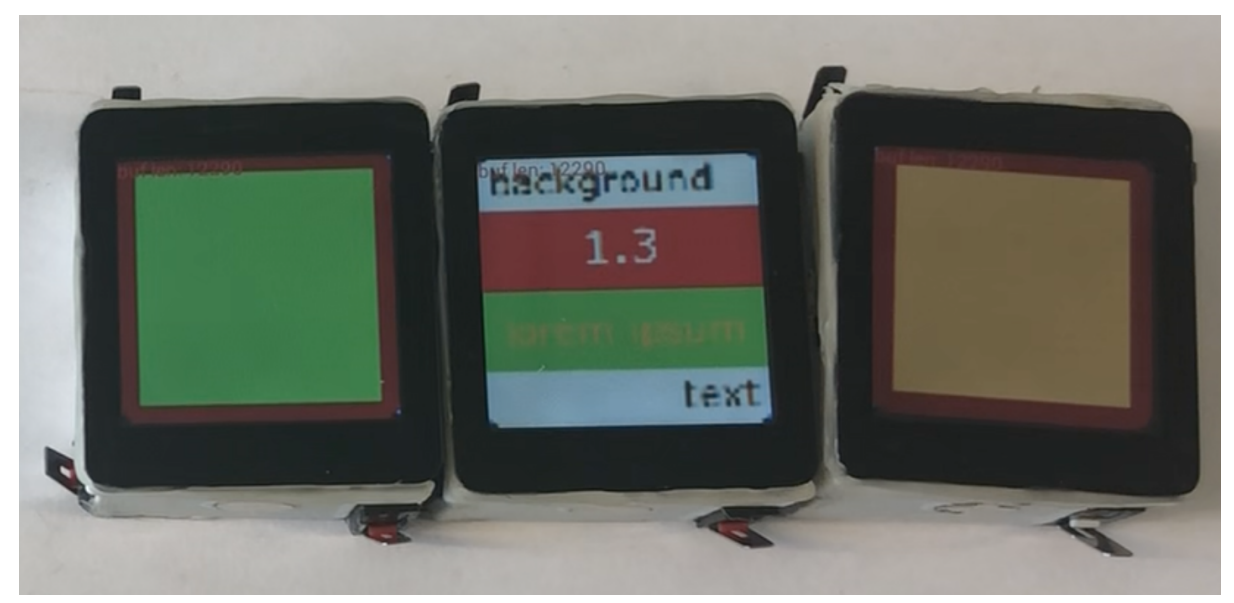

Figure 5.11: Contrast checker cube with colours on the left and right sides. In the centre, a contrast value with a red background for not compliant with colour accessibility standards.

\subsection{Feedback Sessions}

We returned to each design team to demonstrate the prototype and give them an opportunity to use it. We conducted the sessions with designers who had attended the previous workshops and some who had not. We aimed to find out how well the prototype delivers on features discussed in the initial scoping, but also with new designers to gather new feedback not biased from attending the previous workshop. We also aimed to find out how it compares to their current workflows and where they would use it. Finally, we asked if they had any suggestions for improvements or future features that they would like to see.

The sessions were carried out with the designers individually. After a brief interview outlining typical tasks (e.g. exploring complementing colours for a brand/logo/webpages), we presented the participants with the prototype and all its features, followed by a supervised opportunity to become familiar with the prototype. Once familiar, and based on the pre-interview, we asked them to reproduce several of their typical tasks using our device. Each session took 40-60 minutes. During their time using the prototype, we asked them to think aloud. After they had time to explore the prototype fully, we asked follow-up questions, related to the experience, 
where and how they would use it and what improvements they would suggest. As the prototype aimed to support a creative process we took a qualitative approach which valued the subjective thoughts and opinions of the designers. The quality of the hardware was not part of our evaluation; our goal was to record comments on the concept. It was made clear that our hardware was a prototype, and that a final version would have a commercial-product finish.

At the broadcasting organisation, we conducted feedback sessions with seven participants (P1-P7) (M:4 F:3). All were designers working on digital products with one specialising in colour accessibility (P7). At the agency, we conducted feedback sessions with two designers (P8-P9) (M:1 F:1). Each of these designers works with digital marketing materials and brand identities. Of the participants, four had attended the previous workshop (P4-6 and P8). In these feedback sessions we recorded audio and video, which we used for transcription and exploration via thematic analysis to provide the findings presented in the next section.

\subsubsection{User Feedback}

When first playing with the prototype, the participants often commented on how they liked the tangibility of the system. Referring to the side switches, P6 commented "I just really enjoy clicking them together" and "it feels really nice to just browse through the colours." Meanwhile P5 explained how they "really like that it is tactical and I can quickly click and move the colours around" and P4 also really like the feel of the magnets and also added "the size works well, I could imagine using them in a coffee shop ... taking you away from the computer". P1 and P3 each explained that, because of portability, the cubes would be useful for designing in indoors and moving the screen around for testing colours in different lighting.

The participants also explained how it was playful. P1 said, "It's such a nice object to play with" and P7 stated that "it's really nice to have something tangible 
you can play with". Adding to this theme was P6, who explained that it felt entirely personal as it is portable to carry around. P8 said that "it's great that it is small and portable".

When we asked about how the designers would integrate this into the way they currently work, P2 commented how they would use the colour cubes to quickly show others to compare colours with team members and use in the early stages such as workshops. P8 explained how she feels when discovering colours using Pinterest; she is just stealing colours but using this feels more like primary research. P6 said something similar, explaining that she thinks it is suitable for idea generation phases. P1 imagined its uses mainly at the start but still used it throughout. P9 spoke about how he designs a logo in black and white first before adding colour. He explained how he would use this to focus the colour afterwards in order to fill in the logo he has created.

The participants showed how they would start with the brand colours and then build from there. This was a similar process described by P4 who explained: "it would allow me to adjust the palette whenever I need to introduce a new colour". Also referring to the colour contrast checker tool, he explained how he could contrast check as he went along, admitting how it's often left as an afterthought. He also said that it might increase creativity while working. P5 also liked the contrast checker and gave examples of where he needed to use it in his workflows. Also about the contrast checker, P7 said "currently we would have to do that with three different tools" highlighting how it would streamline the process. P7 also said they thought it is excellent for teaching colour theory.

Furthermore, 8/9 of the participants would explain its usefulness for in team workflows. P1 shared with us how he would use it for collaboration in general design-crit sessions as a great way to share ideas. He also explained how it would help to collaborate with non-designers. This idea was also shared with us by P5, P6 and P7, with P7 saying that, "[it is] useful for meetings with stakeholders". P4 also 
mentioned that it would work with non-designers as it is not overwhelming, stating it to be valuable in a multi-discipline team. P6 mentioned that it would get a better response from people and get them to focus. P8 explained how when they design with a client, the client would feel more involved and give them more power over decision making. Finally, not convinced by the group scenario, P3 stated "I would just share colours on a big screen".

When asked about what they liked about the concept, P1 and P9 compared it to Pantone colour swatches and stated that it is nice and intuitive to use. P9 said that they would use it alongside Pantone to quickly reference a colour on printed materials. P4 also mentioned swatches by explaining "What its does perfectly is bridge the gap between looking at Pantone swatches and tweaking colours on your computer." P3 also stated that they imagine them as printed swatches. P2 thought it was 'neat' when seeing the colours update after adjusting the master cube in the chain. They were quick to understand the concept and wanted to get a warmer colour. P4 and P6 were pleased to see the ability to retrieve and send the hex codes to and from the cubes.

We also asked the participants to feedback on any improvements that could be made to support workflows. Of the suggestions made, we saw ones for implementation with the current technology. P1 suggested adding a feature to caption colours alongside the design process. P7 wanted a way to show the text when adjusting the colour after clicking the colours together. Meanwhile P3 thought it would be interesting if it could render patterns and textures in the colours.

Other suggested features would require future hardware solutions. P1 and P6 wanted to be able to scale colours in groups and merge them to be bezel-less. P7 also mentioned getting rid of bezels, but this was because the black outline affected the colour according to colour theory. Linked to the colour grouping, independently $6 / 9$ of the described a system that would link the cubes to colours in a product wireframe to update and test colours in real time. Interestingly, P2 wanted all the sides 
to have a display so that he could quickly reference different colour schemes by rotating the cubes. Combined with this idea, he went on to explain using them as dice for random colour discovery each time a designer rolls the cubes.

P4 mentored a similar thing where changing the orientation would change the colour complementing mode. He also demonstrated using the cubes as a slider based on proximity to nearby cubes to adjust the channels. Another gesture for random colours suggested came from $\mathrm{P} 7$, who demonstrated pulling and taping the cubes to shuffle the colours. Outside the scope of digital design, P3 thought of its use in filmmaking and how filmmakers could make colour storyboards. Finally, $5 / 7$ of the designers wanted a "real-world" colour sampler in the base of the cubes, allowing them to colour pick from a physical object.

Finally, we also took note of some of the criticism and concerns raised about the interface. P6 had concerns about being limited by desk space when using them for personal work and wondered how well it would scale with more people. P2 voiced how it may be hard to convince people who use other tools to switch. P3 did not understand why the cubes need to be connected. P5 explained how he would only use the tool with others and would continue to use digital tools as an individual. P7 thought it was a con because they would need to carry it around as an extra device. P9 stated that it would be good for using with some clients, but he had concerns that less involved clients would see it as devaluing the skills they are paying for because they now need to also find the colours.

\subsection{Discussion and Limitations}

We set out to discover if a tangible medium can benefit interactive digital colour picking. Given this, we spoke with designers to define a framework to conceptualise how to design such a tool for current workflows. This framework outlines discovery, manipulation and presentation. We saw existing tools made the process through 
these stages difficult for designers. We saw that benefits for merging the framework categories into a single interactive system, based on feedback on tangible probes used in workshops with designers. In order to test it, we designed and developed a tangible digital swatch interface then evaluated it with designers.

Our system is influenced by Pantone books and explores an alternative to the potentially costly Pantone based colour design method. In the traditional methods, each colour swatch is set and printed. While this method offers a large choice of quality colour, it suffers from its non-dynamic properties: light naturally fades the colours away and it needs to be changed every two years. In contrast, we exploit the advantage of the digital world: colours remain unchanged on screens, cutting the need for replacement. However, our system constrains the number of swatches designers can use at the same time.

From the evaluation, we saw that our current iteration of the DigiSwatch holds the potential to aid designers in their colour picking process. When evaluated against their current framework, we saw that it mainly supports the discovery and presentation of colours. We outlined the preliminary feedback on colour manipulation and its benefits but also how it can be improved. Many of the designers spoke about its usefulness in the early stages of the process, for instance, when defining new brand colours. Overall, the designers described the tangible aspect of the tool as fun and playful, which could increase their creativity.

Another key theme was collaboration, which is referred to in the discovery and presentation stage. The designers acknowledge that this new tool would be a great way to get a team together to discuss the colours. In the framework, we outlined how tools should support ways of combining colour presentation with discovery and manipulation. Doing so would allow everyone to contribute and make progress together. Another recognised benefit was how they would use the system to involve non-designers, as the system allows for discovery within the constraints of colour 
theory. Plus, they could collaborate and engage with other designers to create a dialogue around why they use that specific colour. This shared experience of picking colours encompasses the three areas of the frameworks and brings to the design an aspect similar to paired programming in software engineering.

We also want to draw attention to findings of the playfulness that came from the tangibility of the system. One participant's remarks suggested that DigiSwatch could aid in designers' general wishes to design away from computers. We also saw mentions that it could enhance creativity and be fun to use because of its tangible elements, for example, clicking the magnets. Another finding associated bringing together the discovery, manipulation and presenting into one system, meaning that all the colours are picked and viewed in the group without going back and forth with GUI tools. This change means that the designers can focus on creating colour schemes together and not individually clicking through menus.

One of the key limitations of the current implementation is manipulation. Many of the designers described the prototype's manipulation controls as intuitive and straightforward, yet they sometimes lacked the colour fidelity with which they were familiar. Despite this, the designers were impressed by how the colours would all update when manipulating a master cube. They also liked that they could simultaneously manipulate colours and dynamically check the contrast value. Overall, we deem manipulation an area with the most exploration ahead. We received useful suggestions from the designers about possible improvement from using the cubes and sliders based on proximity or using the orientations of the cubes to change the mode. We see manipulation as a critical area on which future work can focus to generate different gestures.

We also identify a limitation of the final study in this chapter was our inability to use the prototype in a prolonged design task and therefore missing some further information that could be uncovered from independent use. This limitation came about from a constraint on the amount of time we could spend with the design 
professionals. The designers we worked with were practising full time and often working to deadlines. Also, due to the fidelity of the prototype, we were not able to leave it with them to use in their own time as the set-up in the complex due to the server connections and required the researcher to be present. In future work refining, the prototype to a nearer finished product would go some way to combat these constraints. Future research could leave such a prototype with the designer to experiment in their work practices and report back to the researcher in short meetings. Adopting this method would ensure the designers time is respected and the scenario of the interactions become more realistic.

\subsection{Summary}

This chapter has demonstrated that colour picking for designers is a process that takes much care and attention. Our engagement with teams of designers has highlighted some of the constraints faced when using graphical interfaces for colour picking and demonstrated a map of the process for which technology can support. Using this map, we outlined a framework of the critical elements: discovery, manipulation and presentation.

In testing how we can address these elements, we developed DigiSwatch, a prototype interface that allows individuals and groups to discover and manipulate colours using a modular device for physical interactions. This idea of modular devices allowed us to imitate the interactions seen with the colour swatches with the additional benefits of digital colour pickers. The developed system was made up of screens that were able to sense adjacent colours to provide complementary colour discovery, and each module has a touchscreen for individual colour manipulation.

By designing a colour tool with the framework and obtaining feedback from the 
designers, we saw that it provided a fun and engaging experience with the potential for collaborative work and communication with non-designers and team members. Our finding supports the use of tangible proxies in digital-based processes for playfulness, practicality and reducing the number of external tools needed for design, and the potential to support collaborative workflows across disciplines. Suggestions from the designers point to exploring more diverse physical manipulation techniques.

\subsection{Authors Contributions}

The workshops, user study concept behind the DigiSwatch research was carried out by the author. The implementation of DigiSwatch was supported by a fellow researcher, who used it for a concurrent project, of which, the author also shared a role [22]. This means some technical elements were shared decisions during the building of the prototype. All analysis of the results from designer studies conducted with the DigiSwatch was done entirely by the author. The author planned and ran the study sessions, analysed the results and wrote the paper with feedback from supervisors and other authors.

Although not in the scope of this thesis also of note the authors contributed to the PickCell project. They contributed to the concept and implementation of the Pickcell prototype and design space. They also helped run the workshop sessions and contributed towards the analysis of the results. They also took a role in writing the paper with the other authors, placing a second author. 


\section{Chapter 6}

\section{Discussions, Conclusion and Future}

\section{Work}

In the introduction chapter, we outlined how art and design has seen a great diversity of new digital tools emerge over the last few decades, making it a viable medium for artists and designers to use. The development of these digital tools provide new interaction methods for creatives to express themselves and the digital tools provided hold many new benefits that were not available in traditional methods. As these tools are continually innovating, we considered how to enhance the interactions in this world with novel tools and materials that enable richer tangible interactions with digital art and design.

We highlighted the growing use of tangible interfaces for digital art and design and their use for bringing more realistic experiences to the creative process. To bring about the next wave of innovation, we identified the growing area of deformable interfaces, a field that aimed to address this loss of engagement by combining physical interfaces and actuated change as a medium for interacting with digital worlds.

Therefore, in setting out to explore the world of art and design, we developed deformable interfaces for the many diverse forms of interactions, tools and materials employed by the artists and designers. We studied these elements and learnt the 
importance of their role and functions by working with users. These discussions surfaced information about how they missed the physical interaction they had with their art as the techniques become replaced with modern digital applications.

By learning about physical tools and martial used in these contexts, we have been able to apply these findings to develop new prototypes that aim to merge these two worlds of physical and digital. Throughout this study, we employed the inherent benefits of the deformable interfaces to these scenarios and addressed the design challenges of the art world. The prototypes we developed built upon deformable interface research such as physical actuation, dynamic textures and modular devices. We built and evaluated prototypes with users, and we concluded as to their effectiveness and future potentials. We also highlighted the key contributions they hold for art and design interfaces. This research supports the use of hands-on interactions with such interfaces and a call for similarity to physical counterparts.

Throughout this thesis, we have shown, by how exploring the context of art and design, we can bring about insights for new innovative interfaces. From these insights, we have developed novel forms of interaction that provide a tangible medium for interaction with digital information. In evaluating these prototypes, we show how each chapter has contributed implications for advancing the interfaces for digital art and design. These implications highlight how bringing together tangible rich interactions is a worthwhile endeavour for future advancement of these expressive mediums.

\subsection{Summary of Contributions}

We have explored many research ideas in this thesis around deformable interfaces that drive the development of new art and design interactions. We have presented findings from user research, the devolvement of new prototypes and materials, and 
evaluations of what we developed. The following paragraphs summarises what we did and highlights the significant contributions.

Chapter 1 introduced the growing diversity of tools and materials for digital art and design, outlining a continued innovation in the development of new tangible tools. In order to bring the next wave of innovation in digital art and design tools, our work identifies the potential uses of deformable interfaces that bring about richer interactions. Deformable interfaces allow us to maintain the dynamic benefits of graphical interfaces with the added physicality of tangible interfaces. Moving on to Chapter 2 we reviewed the related literature to identify the gaps to explore in our research. The gaps highlighted the limited research conducted into the use of deformable interfaces in an art and design context. As this gap was relatively underexplored, we saw the opportunity to use art and design as our context to design and develop new deformable interfaces.

In Chapter 3, we explored the different physical art tools needed to build a prototype with actuated controls. This prototype demonstrated how deformable interfaces could be leveraged to form representations of different art tools. The prototype was capable of switching between tangible pots and nibs, so that the user could pick colours from the pots and nib sizes. The controls employed used force sensing to detect interaction, allowing for dipping and squeezing gestures. Participants found using the prototype a fun and enjoyable experience but reported that it did not quite deliver the accuracy of the GUI comparison.

Following the feedback given in the study, we then built a tangible slider with a shape-changing cursor. This cursor would deform in the hands of the user as they slid through selections, allowing for discrete values to communicate a change of shape in the user's hand. This prototype also showed user experience benefits over standard interaction modalities, outperforming GUI outputs by being significantly better for eyes-free control. 
In Chapter 4 we focused our research on painters, moving our research beyond mimicking pot and nibs to realistic paint textures. We gathered findings from studying how painters worked to inform the building of a unique texture-based deformable. In doing this research, we found out about the role textures played in creating artistic works, which are often missed when working in digital mediums.

We designed and developed a texture actuated system that used hydrogel to simulate the mixing of paints on the back of a mobile device. This prototype showed us how we could simulate soft materials and use temperature controlled actuation to enable gestures such as stirring and drawing paints. We also demonstrated how the use of textures on the back of the device enabled the gels to be used in conjunction with the graphical output. Feedback from the artists showed how it was similar to oil paints and the gestures used were comparable to interacting with paints on a palette. In a controlled user study, we confirmed its similarity to oil paints with blind texture tests, which demonstrated how we can simulate the feeling of realworld soft art materials in digital interfaces.

In Chapter 5, we continued with the theme of colour mixing by focusing on digital designers and how they pick a colour for graphic design projects. In this chapter, we presented understandings about how designers work together to make colour schemes. We gathered information about the challenges they face when colour picking and we fielded their suggestions for new tools.

We used the findings to map out a framework that shows how a deformable modular screen could aid these solutions. Using this, we built DigitSwatch, a modular colour swatch system that allows individuals or groups to discover, manipulate and present digital colour. During a feedback session, designers revealed that DigiSwatch addressed the constant by reducing the number of tools needed as well as providing a fun, engaging experience and potential for collaboration. 


\subsection{Discussion}

Throughout this thesis, we have offered contributions to the development of tangible art and design interfaces. Our work can inform future research via the considerations of new deformable elements in the prototypes demonstrated. So, like in the work before us, researchers can harness the designs of our prototypes to inform how they build the next wave of interfaces.

Our work also describes how the art and design context informed the development of these prototypes, yielding findings from the experiences of artists and designers when using their existing tools. Furthermore, some elements of the thesis hold contributions to the field of deformable interfaces, as we have shown new use case and deployment form factors.

\subsubsection{Chosen Context}

The context of art and design has proven an excellent space to study the uses of deformable interfaces. When we look back over the physical tools that artists and designers studied in this thesis already use we can see the physical form enables expression. Whether it was the grasp of the tools, mixing of paints or sharing swatches in a design meeting it?s it all in physical space. Yet the digital world, from a certain perspective, can constrict this expression as it is difficult to emulate such experiences on screens. Therefore using the advantages of deformable interface?s ability to merge the digital and the physical gives such a researcher a great testbed to find new forms of physically expressive activities that deformable could emulate.

This thesis had focused on using deformable interfaces to bring elements of realism to digital art and design experiences. However, because this thesis was predicated on whether using art and design as a diver for developing new deformable 
interfaces there is some case where this may have narrowed focus on other possibles, either using pure graphic approaches, or enhancing peripheral devices on the market, such as styluses or other TUI approaches. These are all of the course areas that have significant bodies of research, as seen in Chapter 2, but some of the user research we present in this thesis could go some way to seed these approaches with new inspiration.

Furthermore, our chosen context has allowed us to offer tools and materials to be used in the future work outside art and design. Rather than offering a fundamental change to the way artists work we aimed to used how the work to inspire deformable interfaces that then enhance then experiences. Throughout the thesis we never focused on changing their process only enhancing the interactions with a deformable innovation. By understanding the users processes first, we were able to bring in tools that support the process, rather than create a fundamental shift to what the user is trying to achieve. We think this has allowed the work to both offer new innovation for art and design, while also offering the HCI community more generalised interaction methods with deformable interfaces. We discuss these later in the chapter in Section 6.2.3.

\subsubsection{Future Art and Design Interfaces}

We have shown through the research presented in this thesis how to develop richer types of tangible interactions for future digital art and design interfaces. These interfaces address the physical contexts of art and design such as tools, paints and swatches. For each area, we developed a prototype that employed elements of actuated controls, dynamic textures or modular devices. Insights from these developed interfaces can be used to support the development of future digital art and design interfaces with richer interactivity. 
In Chapter 3, we showed how to use physical actuation to switch between controls that take the form of tools seen in traditional art. We showed how areas could submerge to make pots for picking colours or emerge for shaping nib sizes. Future interfaces might consider the use of actuated controls to add tangible elements for enhancing digital tools, such as graphics tablets. This addition would upgrade such tools beyond their existing flat screen formats, as there would be an added dimension of physicality. It would also take advantage of actuation, allowing them to maintain versatility, as tools will be able to change their physical form on demand. Doing so may provide a more enjoyable user experiences, and based on our evaluations, providing that the work is done, the make the interactions are more accurate, like that of a touchscreen.

Our exploration using a deformable slider may offer one possible way to add this accuracy to the sensels missed in the force-based interaction. We demonstrated a way of providing in hand feedback of shape-changing on a slider thumb. Our early results showed that there was some viability over traditional mediums such as sound, vibration and outperformed GUI feedback. This potentially contributes a new modality for feedback on a sensel-based interface with an additional layer of feedback tacking changes. Within the focus of this thesis, such size change could be mapped to nib and brush sizes when an artist uses digital art software. This might improve the issues of accuracy discovered in the Chapter 3's first prototype.

In Chapter 4, we demonstrated the use of hydrogel for paint simulation in digital art interfaces and hows its dynamic properties allowed it to support a set of gestures associated with mixing real paints. These types of dynamic materials can be employed as added layers to an interface and provide textured sensations. This layering will make using the interface more realistic and engaging based on its demonstrated ability to represent soft materials like oil paint, and how the temperature actuated change provides gestures for mixing paint. We also showed it as a back of the device attachment, an element that suggests the pairing of textures with 
graphical output for harmony between digital art and physical interface. Feedback shows how these elements made for realistic interactions and how future developments can draw on the use of textures as a sensual layer between the interface and the artist. More speculatively we suggested that the use of textures would make digital painting more expressive and creative.

Finally, Chapter 5 integrated the use of modular device concepts with digital colour picking; this contributed a digital colour swatch system named DigiSwatches. As we see, when the art studio moves towards more portable form factors (e.g. iPad Pro and Apple Pencil combinations), the possible concepts for phones and tablets to break apart into modular elements enters the realm of art and design. We showed how a prototype device capable of modularity and dynamic output provides the ability for designers to browse digital colours like analogue swatches. The prototype also combined tools previously separated in existing interfaces, which combined dynamic colour exploration with manipulation and contrast inspection. Designers highlighted that this type of tool makes for a more playful and engaging experience and speculate its potential for more collaborative design meetings. Therefore, our research suggests its viability as a future colour picking interface or as a modular component of an existing workstation.

Overall, each of the thesis chapters have illustrated the types of rich interactions that can inform future art and design interfaces when employing aspects of deformable interfaces. We have contributed technical prototypes that provide developers with a look into the future of what tools can become, providing them with new considerations such as adding actuation to controls, the use of textures to add layers of physical sensations, and how to modularise screens for physical browsing and sharing colours. These are all possibilities for what digital art interactions can become when researching richer technologies and building with consideration for the physical elements of art and design practices. It demonstrates the values of hands-on interfaces and how they can be a more playful and engaging experience. 


\subsubsection{Implications for Deformable Interfaces}

Although rooted in the context of art and design, our work also holds some implication for the use of future deformable interfaces. Some of the elements of the new prototypes we build hold novel findings that contribute to this broader field of research for both extending technical knowledge and demonstrating their use in context.

The context of art and design has proven an excellent space to study the uses of deformable interfaces. When we look back over the physical tools that artists and designers studied in this thesis already use we can see the physical form enables expression. Whether it was the grasp of the tools, mixing of paints or sharing swatches in a design meeting it?s it all in physical space. Yet the digital world, from a certain perspective, can constrict this expression as it is difficult to emulate such experiences on screens. Therefore using the advantages of deformable interface?s ability to merge the digital and the physical gives the researcher a great test bed to find new forms of physically expressive activities that deformable could emulate. We encourage not only more deformable research to be done in the context of art and design but other contexts with large amounts of physical expression, as it offers an opportunity to challenge what is possible and seek out new materials and technical implementation.

We have demonstrated a device capable of using physical actuation to change its form with the addition of force-based sensing for interaction. This device employed emerging and submerging sensel elements to represent realistic tools seen in past work, but the use of force-based control added a new layer of interaction for the sensel extending the input possibilities. The feedback on the sensel-based prototype lead us to make a slider capable of using physical actuation of a cursor in a user's hand to provide a haptic output on physical sliders. This in-hand deformation mixed with the use of the slider on its track, brings about a new type of concurrent input and shape-changing output extending the fidelity haptic feedback 
to include physical actuation. The study showed good evidence for being better than GUI output and comparable to sound and vibration modalities. This findings can inform future deformable work with actuated interfaces using sensels with a mix of interaction modalities.

A key challenge seen in the deformable prototypes shown through Chapters 3 and 4 was the actuation time and deciding where it may hinder the user in future. While the device offers benefits for user experience there?s always going to be a time lag when the devices deform to show their new forms. For our first painting palette, deformable users needed to wait for the servo motors the most in order to use the different controls. Then when using the slider, although this actuated in transit was still not as instant and its comparison outputs, such as sound or light. In a GUI both these controls would have instantly changed and therefore of other that some experience benefits the actuation in prolonged could offer a significant time loss. Then in Chapter 4, the user needs to wait before the temperature is reached and the gel the mix viscosity. However, in this scenario, the time taken to change is part of the mixing experience and more akin to paint in reality. Therefore, the gel action offered a more unique experience much further removed from using a GUI. As the context we explored was art and design there?s strong augment that physical experience plays a role in creative expression. But as this work is generalised to other contexts and deployed in future interfaces, designers will need to consider when the benefits of experiences using a deformable benefit the context over more responsive GUIs.

We also contribute new perspectives for using the dynamic textures. In Chapter 4, our work has extended knowledge on the use of hydrogel, showing its use as a stiffness actuated gel to simulate soft materials on mobile devices. The use of this enables touching gestures akin to touching liquid-like materials in the real world. We showed how using this on the back-of-device scenario enabled it to pair the physical textures with digital output. This chemical is a new possibility that 
is still under exploration as it could be used for many other things in the field of human-computer interaction.

For example much of what we have done to simulate the tools and materials for the art and design world could be used in other contexts where there needs to be a degree of realism for the user. Firstly, video games would be an excellent context for the tools presented to be adapted to as video game designers go to great lengths to simulate a realistic experience for the player, much as we have done for an artist. Taking the interaction techniques presented in this thesis and using them for video games and virtual worlds could make these experiences much more immersive for the players.

Furthermore, another example of contexts with the need for simulations is medical or military training. Both these contexts incur high risk if not adequately trained for correctly but then equally are difficult to simulate the same high-risk environment in a digital context to democratise allowing new people to enter the profession. Materials such as the hydrogel offer a starting point for making sensations of fake human tissue that could be paired with digital output of sounds and images of hospitals and battlefields. Having such tools in a form factor as easy to distribute as computers and tablets would offer more opportunity to people looking to enter the profession.

We have also extended the knowledge of shape-changing interaction devices with the use of modular screens. This work showed how spatial deformable devices could support the physical browsing of digital information across a breakable and re-configurable set of screens. This possibility demonstrated how shape-change and gestures dictated how and which type of information was shown and what functions were accessed. Furthermore, we can already begin to see the broader implications of using a modular approach for mobile devices and mapping pre-existing work to design space for the HCI community. For instance, the PickCells project [22], which we led with collaborators, has shared a hardware development process with 
DigiSwatches. The project outlines the broader implications of modular screens and the design they potentially bring to the deformable screen field. Our contextdriven approach in this thesis surfaced good evidence for the professional user's needs in such an approach to interfaces.

\subsection{Limitations and Future Work}

Throughout this thesis, we have presented research that offers advances for the field of art and design interfaces. From gathering an understanding of the creative domain, we have developed prototypes with physical actuation, texture, and modularity. We have highlighted how the concepts might inform the development of these future interfaces. Based on our contributions, we outline some of the current limitations of the research with and some future work we deem to be potential next steps. These contributions have implications for digital art and design interfaces and some suggestions to the broader field of deformable interfaces.

In identifying the scope of this thesis, we limited our study of art and design to the tools, paints and swatches in the physical world. This scope gave us clear challenges for which to design our prototypes and are each are unexplored fields when considering new interfaces. Our work has shown how the study of each of these areas can produce richer interactions for artists and designers. Hence, future work should consider other key processes and build tools as we have to broaden the research of using deformable interfaces for art and design.

In Chapter 3, we showed a tool that could transform the physical shape into paint pot colour controls and emerged nib controls. In order to detect the interactions, we used force sensors and foam to cover them for a limited textured sensation. This sensing technique was new to sensel-based interactions but was possibly a limited precision. Also, unlike the later slider prototype or the hydrogel, it was unable to change and hold its form to communicate the state the user left. 
Furthermore, materials such as hydrogel (which is explored in Chapter 4) have the potential to enhance what is capable of sensel-based actuation in deformable controls. Sensels are a suitable physical analogue to the digital pixel and a future device can bring together soft materials like hydrogel with high fidelity sensel interaction for a vision of pots in screens with liquid feeling of paints. Yet, it still requires a significant challenge to use these types of materials in a sensel actuated display, as moving soft materials offers more variables and therefore a challenge to control an outcome. In turn, this limits our work to study physical actuators and texture independently.

The hydrogel we used to make paint textures on the back of mobile devices itself has limitations around freedom of placement on the device. In future, it would be ideal to have the gel on the front of the screens to achieve greater parity with what is happening on the screen and the texture changes taking place. To achieve this parity, we would need future work to advance the chemistry to keep the gel transparent when fully actuated. Placing the gel on the front of the screen would also need significant engineering effort because the touchscreen would now need to heat and cool the gel actively.

One possible direction for solving screen obscurity and increased fidelity could include using high-powered LEDs to heat specific areas of the gel. This use of LED would increase the fidelity and could act as a high power display to shine colours beyond the gel. The questions then would remind us: how do we cool the gel after it's heated? In order to achieve the mixing interaction, the LEDs would be hot and would cool more slowly than the existing technique. Currently, to the best of our knowledge, Peltiers remain the most effective solution to this issue. Future work could explore alternative cooling methods, bringing them to the fidelity achieved on the mobile scale.

Our final suggestion for further studies with hydrogel would be studying its use with virtual and augmented reality. Doing so many surpass the need for the gel to 
be placed over the screen as the graphics would be overlaid in front of the user's field of view by a device such as an Oculus Rift ${ }^{1}$ or Microsoft Hololens ${ }^{2}$. Such technology would then allow for a whole manner of textures, so long as a user can perceive them in relation to what they see in the virtual world. This relationship would open a new challenge such as tracking the change in the gel to update the display and then maintaining synchronisation. Art is no stranger to virtual reality so exploring the use of textures may further enhance these new technologies, as we showed with mobile devices.

The modular screen system used in Chapter 5 that made up DigiSwatch is currently able to detect each other's presence based on adjacent magnetic attachment. This method gives the formation of the shape of the screen the primary control over the displayed information on each of the screens. The discussion with the users and their suggestions provide future directions to add motion or even proximity detection as an added interactions for control of content. Adding in these controls to modular screens could also offer future enhancements of physical manipulations of the data they display.

Furthermore, we suggest not only giving the user modular controls, but also the system. Currently, only the user can re-configure the screens and then the displays are updated based on the configurations made. Future work could also explore giving this control to the system, so that it might dynamically reconfigure without the user's input. This use could open up the opportunity for the system to suggest changes to the user, such as moving a module to a different adjacent colour or suggesting a better contrast. Doing this work would require an engineering solution for modules to move independently and research to find a balance of user controls and system autonomy.

Our work has focused on gathering requirements and building initial prototypes

\footnotetext{
${ }^{1}$ Oculus Rift: https: //WwW. oculus. com

${ }^{2}$ Microsoft Hololens: https: //WWW . microsoft. com/en-us/hololens
} 
in order to research early feedback and test the feasibility of the prototypes. Future work should look to developing a more robust version of the developed prototypes in order to integrate them into the workflow of artists and designers. Doing this type of study would help to gather data on their long-term effect on artists and designers. Doing so will allow us to see if there is a significant improvement to their workflow. In order to achieve this vision, more expertise from the engineering field could overcome some of the limitations UX designers face when trying to build these complex systems.

Finally, we end on a discussion with lessons for future HCI researchers in the space of deformable interfaces. One lesson we learnt was making use of research collaborations across the academic communities. During Chapter 5 we drew on expertise from chemists, and by doing so enhanced the prototype we wanted to build giving us new opportunities we would not have been able to reach if we only used skills of computer science. Therefore based on this experience, it is recommended to seek knowledge and support from other research disciplines as more collaborations would help HCI researchers achieve unique interaction techniques.

Building upon this, we encourage researchers of deformable interfaces to stretch out of their fields and explore the context for the deformable they develop. For us, the use of art and design offered diverse opportunities for discovering new forms of shape-changing interfaces with each one relating to a real-world interaction it can map to in the digital. As the field deformable interface matures using a context to inform new possibilities or allow existing methods to map to use-cases, such as actuation techniques show in Chapter 3.

Furthermore, this could be done via either bottom-up where the users and the context directly inform what is built or with top-down questions where developed interfaces are taken into a context and tested for their relevance. Using a mix of the two would serve to maintain the imagination of the research with a tech top-down, while the bottom-up approach would serve as an inspiration to those looking for 
something user lead. We think both of these types of approaches will help mature the field and make it more relevant to the wider context of HCI as research can be better mapped to interactions with existing methods as an alternative to GUIs. It would help move deformable interfaces close to the bridge between academia and industry, aiding engineers and developers see relevant contexts for deformable and where they can start to roll them out to everyday users.

A final note to future $\mathrm{PhD}$ researchers entering the field of deformable interface research. Throughout the process of researching the work that appears in this thesis, the more we left the confines of our lab the more inspirations we gathered. Deformable interfaces are physical things and are part of our world and therefore we encourage others to explore that world. Along the way, you will find users to learn from like the artist you have seen in our research. You will meet people that can help you achieve things beyond bit and bytes as the chemists that showed us how to create the gel screen that feels like paint. We encourage any $\mathrm{PhD}$ researchers entering deformable research to take their ideas gathered from user-centred-design and similar approaches and share it with other disciplines so that might just make the previous reserved for the digital happen in the physical.

\subsection{Concluding Remarks}

As the market leaders in the field of interaction design continue to develop the next wave of art and design products, we still only see consideration for static tangibles, such as styles or tablets. Yet, as this thesis has observed, when we investigate the physical art world, there are many tools and materials that have yet to be presented in digital worlds. To consider bringing such interactions to the digital world, we explored using the new deformable interfaces capable of providing such dynamic richness. We have proposed our vision to unlock these new possibilities using such 
technology as actuated interfaces, dynamic textures and modular displays. We encourage others to join in pursuing this vision and bringing artist and designers closer to their work in more engaging interactions.

Our research had already begun to demonstrate this first step into such a future. It has shown us how to harness the current state of deformable interfaces for tools, paint and swatches. Beyond the progress presented in this thesis, future technology will continue to give researchers richer interactions to explore. The continuation of this work will give engineers and developers an abundance of possibilities built for digital creatives, aiding the world of art and design for all to enjoy. 


\title{
Appendix A
}

\section{Contributing Publications}

Here we list the work from the thesis that was published in peer-reviewed international conferences.

(P1) Cameron Steer, Jennifer Pearson, Simon Robinson, and Matt Jones. Deformable paint palette: Actuated force controls for digital painting. In Proceedings of the 2017 CHI Conference Extended Abstracts on Human Factors in Computing Systems, CHI EA '17, pages 2936-2943, New York, NY, USA, 2017. ACM.

\begin{abstract}
This work-in-progress presents early findings on using deformable interfaces with force input to enhance interactions with digital painting applications. Our prototype uses deformation to submerge controls to represent paint pots, and emerge controls to represent nibs. These controls can then be pressed or squeezed to change the colour or size used for painting. Our preliminary study evaluates the prototype against a tablet. Early findings show participants were quicker and more accurate using the tablet, and found mapping of their input force to response unclear when using the prototype. Despite this, participants enjoyed using the deformable interface, stating that tangibility was useful for the use experience. We report these results and offer suggestions for improvement of the deformable interface.
\end{abstract}


Author's contribution The concept and implementation behind this research was mine. I planned and ran the study sessions, analysed the results and wrote the paper with feedback from other authors.

(P2) Cameron Steer. Designing mobile deformable controls for creation of digital art. In Proceedings of the 19th International Conference on HumanComputer Interaction with Mobile Devices and Services. New York, NY, USA, 2017. ACM.

\begin{abstract}
My research explores the design and development of deformable controls and shape displays for mobile devices. My significant work so far has been set in the context of tools for creating digital art. I have been studying how we might design and develop deformable interfaces that support interaction with digital art applications. Along with how to bring elements of realistic painting to the digital experience through physical mobile controls. Part of this is designing and prototyping physical hardware interfaces and then evaluating them using HCI research methods in user studies and interviews. This extended abstract outlines my research aims, progress so far, and my future work and direction.
\end{abstract}

Author's contribution This Doctoral Consortium extended abstract was presented in MobileHCI 2017 DC. Here session I participated in a group consortium and displayed a poster based on the paper. The concepts and writing of the paper were done by me, with feedback and advice from colleagues and supervisors.

(P3) Cameron Steer, Simon Robinson, Jennifer Pearson, Deepak Sahoo, Ian Mabbett, and Matt Jones. A liquid tangible display for mobile colour mixing. In To appear in the proceedings of the 20th International Conference on HumanComputer Interaction with Mobile Devices and Services, New York, NY, USA, 2018. ACM. 
Abstract. Digital painting is an increasingly popular medium of expression for many artists, yet when compared to its traditional equivalents of physical brushes and viscous paint it lacks a dimension of tangibility. We conducted observations and interviews with physical and digital artists, which gave us a strong understanding of the types of interactions used to create both physical and digital art, and the important role tangibility plays within these experiences. From this, we developed a unique liquid-like tangible display for mobile, digital colour mixing. Using a chemical hydrogel that changes its viscosity depending on temperature, we are able to create some resemblances to the feeling of mixing paint with a finger. This paper documents the information gathered from working with artists, how this process informed the development of a mobile painting attachment, and an exploration of its capabilities. After returning with our prototype, we found that it provided artists with sensations of oil and acrylic paint mixing and also successfully mimicked how paints are laid out on a paint palette.

Author's contribution The concept and implementation behind this research was mine. Support was provided by Swansea Chemistry Department to source materials and demonstrate how to safely prepare hydrogel. I planned and ran the study sessions, analysed the results and wrote the paper with feedback from supervisors and other authors. Deepak Sahoo as an expert on using new materials in HCI and Ian Mabbett who help with sourcing the Hydrogel.

(P4) Alix Goguey, Cameron Steer, Andrés Lucero, Laurence Nigay, Deepak Sahoo, Céline Coutrix, Anne Roudaut, Sriram Subramanian, Yutaka Tokuda, Timothy Neate, Jennifer Pearson, Simon Robinson, and Matt Jones. Pick-cells: A physically reconfigurable cell-composed touchscreen. In CHI Conference on Human Factors in Computing Systems Proceedings, CHI '19, pages 273:1273:14, New York, NY, USA, 2019. ACM.

Abstract. Touchscreens are the predominant medium for interactions with 
digital services; however, their current fixed form factor narrows the scope for rich physical interactions by limiting interaction possibilities to a single, planar surface. In this paper we introduce the concept of PickCells, a fully re- configurable device concept composed of cells, that breaks the mould of rigid screens and explores a modular system that affords rich sets of tangible interactions and novel across- device relationships. Through a series of co-design activities - involving HCI experts and potential end-users of such systems - we synthesised a design space aimed at inspiring future research, giving researchers and designers a framework in which to explore modular screen interactions. The design space we propose unifies existing works on modular touch surfaces under a general framework and broadens horizons by opening up unexplored spaces providing new interaction possibilities. In this paper, we present the Pick-Cells concept, a design space of modular touch surfaces, and propose a toolkit for quick scenario prototyping.

Author's contribution I contributed to the concept and implementation of the Pickcell prototype and design space. I helped run the workshop sessions and contributed towards the analysis of the results. I also took a small role in writing the paper with the other authors. 


\section{Bibliography}

[1] Ahmed Al Maimani and Anne Roudaut. Frozen suit: Designing a changeable stiffness suit and its application to haptic games. In Proceedings of the 2017 CHI Conference on Human Factors in Computing Systems, CHI '17, pages 2440-2448, New York, NY, USA, 2017. ACM.

[2] Jason Alexander, John Hardy, and Stephen Wattam. Characterising the physicality of everyday buttons. In Proceedings of the Ninth ACM International Conference on Interactive Tabletops and Surfaces, ITS '14, pages 205-208, New York, NY, USA, 2014. ACM.

[3] Jason Alexander, Anne Roudaut, Jurgen Steimle, Kasper Hornbæk, Miguel Bruns Alonso, Sean Follmer, and Timothy Merritt. Grand challenges in shape-changing interface research. In Proceedings of the 2018 CHI Conference on Human Factors in Computing Systems, CHI '18, pages 299:1299:14, New York, NY, USA, 2018. ACM.

[4] Bill Baxter, Vincent Scheib, Ming C. Lin, and Dinesh Manocha. Dab: Interactive haptic painting with 3d virtual brushes. In ACM SIGGRAPH 2005 Courses, SIGGRAPH '05, New York, NY, USA, 2005. ACM.

[5] Joanna Berzowska and Marcelo Coelho. Kukkia and vilkas: Kinetic electronic garments. In Proceedings of the Ninth IEEE International Symposium on Wearable Computers, ISWC '05, pages 82-85, Washington, DC, USA, 2005. IEEE Computer Society. 
[6] Paul Cairns and Anna L Cox. Research methods for human-computer interaction, volume 12. Cambridge University Press Cambridge, 2008.

[7] Stuart K. Card, Jock D. Mackinlay, and George G. Robertson. A morphological analysis of the design space of input devices. ACM Trans. Inf. Syst., 9(2):99-122, 1991.

[8] Angela Chang and Conor O'Sullivan. Audio-haptic feedback in mobile phones. In CHI '05 Extended Abstracts on Human Factors in Computing Systems, CHI EA '05, pages 1264-1267, New York, NY, USA, 2005. ACM.

[9] Jean Chevalier and Alain Gheerbrant. A dictionary of symbols. Blackwell Oxford, 1994.

[10] Koeun Choi, Hyunjoo Song, Kyle Koh, Jinwook Bok, and Jinwook Seo. Peek-a-view: Smartphone cover interaction for multi-tasking. In Proceedings of the 2016 CHI Conference on Human Factors in Computing Systems, CHI '16, pages 4658-4662, New York, NY, USA, 2016. ACM.

[11] Loraine Clarke and Eva Hornecker. Designing and studying a multimodal painting installation in a cultural centre for children. In Fourth International Workshop on Physicality, pages 28-31, 2012.

[12] Marcelo Coelho, Hiroshi Ishii, and Pattie Maes. Surflex: A programmable surface for the design of tangible interfaces. In CHI'08 Extended Abstracts on Human Factors in Computing Systems, CHI EA '08, pages 3429-3434, New York, NY, USA, 2008. ACM.

[13] Ashley Colley, Aki Leinonen, Meri-Tuulia Forsman, and Jonna Häkkilä. Ems painter: Co-creating visual art using electrical muscle stimulation. In Proceedings of the Twelfth International Conference on Tangible, Embedded, and Embodied Interaction, TEI '18, pages 266-270, New York, NY, USA, 2018. ACM. 
[14] Patricia I. Cornelio Martinez, Emanuela Maggioni, Kasper Hornbæk, Marianna Obrist, and Sriram Subramanian. Beyond the libet clock: Modality variants for agency measurements. In Proceedings of the 2018 CHI Conference on Human Factors in Computing Systems, CHI '18, pages 541:1541:14, New York, NY, USA, 2018. ACM.

[15] Christian Corsten, Christian Cherek, Thorsten Karrer, and Jan Borchers. Hapticase: Back-of-device tactile landmarks for eyes-free absolute indirect touch. In Proceedings of the 33rd Annual ACM Conference on Human Factors in Computing Systems, CHI '15, pages 2171-2180, New York, NY, USA, 2015. ACM.

[16] Christian Corsten, Bjoern Daehlmann, Simon Voelker, and Jan Borchers. Backxpress: Using back-of-device finger pressure to augment touchscreen input on smartphones. In Proceedings of the $2017 \mathrm{CHI}$ Conference on $\mathrm{Hu}$ man Factors in Computing Systems, CHI '17, pages 4654-4666, New York, NY, USA, 2017. ACM.

[17] Panteleimon Dimitriadis and Jason Alexander. Evaluating the effectiveness of physical shape-change for in-pocket mobile device notifications. In Proceedings of the SIGCHI Conference on Human Factors in Computing Systems, CHI '14, pages 2589-2592, New York, NY, USA, 2014. ACM.

[18] Tom Djajadiningrat, Stephan Wensveen, Joep Frens, and Kees Overbeeke. Tangible products: redressing the balance between appearance and action. Personal and Ubiquitous Computing, 8(5):294-309, 2004.

[19] Aluna Everitt and Jason Alexander. Polysurface: A design approach for rapid prototyping of shape-changing displays using semi-solid surfaces. In Proceedings of the 2017 Conference on Designing Interactive Systems, DIS '17, pages 1283-1294, New York, NY, USA, 2017. ACM. 
[20] Aluna Everitt, Faisal Taher, and Jason Alexander. Shapecanvas: An exploration of shape-changing content generation by members of the public. In Proceedings of the 2016 CHI Conference on Human Factors in Computing Systems, CHI'16, pages 2778-2782, New York, NY, USA, 2016. ACM.

[21] Sean Follmer, Daniel Leithinger, Alex Olwal, Akimitsu Hogge, and Hiroshi Ishii. inform: Dynamic physical affordances and constraints through shape and object actuation. In Proceedings of the 26th Annual ACM Symposium on User Interface Software and Technology, UIST '13, pages 417-426, New York, NY, USA, 2013. ACM.

[22] Alix Goguey, Cameron Steer, Andrés Lucero, Laurence Nigay, Deepak Sahoo, Céline Coutrix, Anne Roudaut, Sriram Subramanian, Yutaka Tokuda, Timothy Neate, Jennifer Pearson, Simon Robinson, and Matt Jones. Pickcells: A physically reconfigurable cell-composed touchscreen. In CHI Conference on Human Factors in Computing Systems Proceedings, CHI '19, pages 273:1-273:14, New York, NY, USA, 2019. ACM.

[23] Antonio Gomes, Andrea Nesbitt, and Roel Vertegaal. Morephone: A study of actuated shape deformations for flexible thin-film smartphone notifications. In Proceedings of the SIGCHI Conference on Human Factors in Computing Systems, CHI '13, pages 583-592, New York, NY, USA, 2013. ACM.

[24] Antonio Gomes, Calvin Rubens, Sean Braley, and Roel Vertegaal. Bitdrones: Towards using $3 \mathrm{~d}$ nanocopter displays as interactive self-levitating programmable matter. In Proceedings of the $2016 \mathrm{CHI}$ Conference on $\mathrm{Hu}$ man Factors in Computing Systems, CHI '16, pages 770-780, New York, NY, USA, 2016. ACM.

[25] Daniel Groeger, Elena Chong Loo, and Jurgen Steimle. Hotflex: Post-print customization of $3 \mathrm{~d}$ prints using embedded state change. In Proceedings of the 2016 CHI Conference on Human Factors in Computing Systems, CHI 
'16, pages 420-432, New York, NY, USA, 2016. ACM.

[26] Erik Gronvall, Sofie Kinch, Marianne Graves Petersen, and Majken K. Rasmussen. Causing commotion with a shape-changing bench: Experiencing shape-changing interfaces in use. In Proceedings of the SIGCHI Conference on Human Factors in Computing Systems, CHI '14, pages 2559-2568, New York, NY, USA, 2014. ACM.

[27] John Hardy, Christian Weichel, Faisal Taher, John Vidler, and Jason Alexander. Shapeclip: Towards rapid prototyping with shape-changing displays for designers. In Proceedings of the 33rd Annual ACM Conference on Human Factors in Computing Systems, CHI '15, pages 19-28, New York, NY, USA, 2015. ACM.

[28] Chris Harrison and Scott E. Hudson. Providing dynamically changeable physical buttons on a visual display. In Proceedings of the SIGCHI Conference on Human Factors in Computing Systems, CHI '09, pages 299-308, New York, NY, USA, 2009. ACM.

[29] Nikolaj Haulrik, Rasmus M. Petersen, and Timothy Merritt. Cadlens: Haptic feedback for navigating in 3d environments. In Proceedings of the 2017 ACM Conference Companion Publication on Designing Interactive Systems, DIS '17 Companion, pages 127-131, New York, NY, USA, 2017. ACM.

[30] Liang He, Gierad Laput, Eric Brockmeyer, and Jon E. Froehlich. Squeezapulse: Adding interactive input to fabricated objects using corrugated tubes and air pulses. In Proceedings of the Eleventh International Conference on Tangible, Embedded, and Embodied Interaction, TEI '17, pages 341-350, New York, NY, USA, 2017. ACM.

[31] Fabian Hemmert, Susann Hamann, Matthias Löwe, Josefine Zeipelt, and Gesche Joost. Shape-changing mobiles: Tapering in two-dimensional deformational displays in mobile phones. In CHI' 10 Extended Abstracts on 
Human Factors in Computing Systems, CHI EA '10, pages 3075-3080, New York, NY, USA, 2010. ACM.

[32] Seongkook Heo and Geehyuk Lee. Force gestures: Augmented touch screen gestures using normal and tangential force. In CHI'11 Extended Abstracts on Human Factors in Computing Systems, CHI EA '11, pages 1909-1914, New York, NY, USA, 2011. ACM.

[33] Seongkook Heo and Geehyuk Lee. Indirect shear force estimation for multipoint shear force operations. In Proceedings of the SIGCHI Conference on Human Factors in Computing Systems, CHI '13, pages 281-284, New York, NY, USA, 2013. ACM.

[34] Yuki Hirobe, Takumi Yoshida, Shinobu Kuroki, Kouta Minamizawa, Katsunari Sato, and Susumu Tachi. Colorful touch palette. In ACM SIGGRAPH 2010 Emerging Technologies, SIGGRAPH '10, pages 10:1-10:1, New York, NY, USA, 2010. ACM.

[35] Eve Hoggan and Stephen A. Brewster. Crosstrainer: Testing the use of multimodal interfaces in situ. In Proceedings of the SIGCHI Conference on Human Factors in Computing Systems, CHI '10, pages 333-342, New York, NY, USA, 2010. ACM.

[36] Eve Hoggan, Andrew Crossan, Stephen A. Brewster, and Topi Kaaresoja. Audio or tactile feedback: Which modality when? In Proceedings of the SIGCHI Conference on Human Factors in Computing Systems, CHI '09, pages 2253-2256, New York, NY, USA, 2009. ACM.

[37] David Holman and Roel Vertegaal. Organic user interfaces: Designing computers in any way, shape, or form. Commun. ACM, 51(6):48-55, 2008.

[38] Eva Hornecker and Jacob Buur. Getting a grip on tangible interaction: A framework on physical space and social interaction. In Proceedings of the 
SIGCHI Conference on Human Factors in Computing Systems, CHI '06, pages 437-446, New York, NY, USA, 2006. ACM.

[39] Caroline Hummels, Kees C. J. Overbeeke, and Sietske Klooster. Move to get moved: a search for methods, tools and knowledge to design for expressive and rich movement-based interaction. Personal and Ubiquitous Computing, 11(8):677-690, 2007.

[40] Sungjae Hwang, Myungwook Ahn, and Kwang-yun Wohn. Maggetz: Customizable passive tangible controllers on and around conventional mobile devices. In Proceedings of the 26th Annual ACM Symposium on User Interface Software and Technology, UIST '13, pages 411-416, New York, NY, USA, 2013. ACM.

[41] Hiroshi Ishii. The tangible user interface and its evolution. Commun. ACM, 51(6):32-36, 2008.

[42] Hiroshi Ishii, Dávid Lakatos, Leonardo Bonanni, and Jean-Baptiste Labrune. Radical atoms: Beyond tangible bits, toward transformable materials. interactions, 19(1):38-51, 2012.

[43] Hiroshi Ishii, Daniel Leithinger, Sean Follmer, Amit Zoran, Philipp Schoessler, and Jared Counts. Transform: Embodiment of radical atoms at milano design week. In Proceedings of the 33rd Annual ACM Conference Extended Abstracts on Human Factors in Computing Systems, CHI EA '15, pages 687-694, New York, NY, USA, 2015. ACM.

[44] Hiroshi Ishii, Sandia Ren, and Phil Frei. Pinwheels: Visualizing information flow in an architectural space. In CHI 'O1 Extended Abstracts on Human Factors in Computing Systems, CHI EA '01, pages 111-112, New York, NY, USA, 2001. ACM.

[45] Hiroshi Ishii and Brygg Ullmer. Tangible bits: Towards seamless interfaces 
between people, bits and atoms. In Proceedings of the ACM SIGCHI Conference on Human Factors in Computing Systems, CHI '97, pages 234-241, New York, NY, USA, 1997. ACM.

[46] Ghita Jalal, Nolwenn Maudet, and Wendy E. Mackay. Color portraits: From color picking to interacting with color. In Proceedings of the 33rd Annual ACM Conference on Human Factors in Computing Systems, CHI '15, pages 4207-4216, New York, NY, USA, 2015. ACM.

[47] Sungjune Jang, Lawrence H. Kim, Kesler Tanner, Hiroshi Ishii, and Sean Follmer. Haptic edge display for mobile tactile interaction. In Proceedings of the 2016 CHI Conference on Human Factors in Computing Systems, CHI '16, pages 3706-3716, New York, NY, USA, 2016. ACM.

[48] Yvonne Jansen, Pierre Dragicevic, and Jean-Daniel Fekete. Tangible remote controllers for wall-size displays. In Proceedings of the SIGCHI Conference on Human Factors in Computing Systems, CHI '12, pages 2865-2874, New York, NY, USA, 2012. ACM.

[49] Yvonne Jansen, Thorsten Karrer, and Jan Borchers. Mudpad: A tactile memory game. In ACM International Conference on Interactive Tabletops and Surfaces, ITS '10, pages 306-306, New York, NY, USA, 2010. ACM.

[50] Yvonne Jansen, Thorsten Karrer, and Jan Borchers. Mudpad: Tactile feedback and haptic texture overlay for touch surfaces. In ACM International Conference on Interactive Tabletops and Surfaces, ITS '10, pages 11-14, New York, NY, USA, 2010. ACM.

[51] Hsin-Liu (Cindy) Kao, Miren Bamforth, David Kim, and Chris Schmandt. Skinmorph: Texture-tunable on-skin interface through thin, programmable gel. In Proceedings of the 2018 ACM International Symposium on Wearable Computers, ISWC '18, pages 196-203, New York, NY, USA, 2018. ACM. 
[52] Daniel F. Keefe, Daniel Acevedo Feliz, Tomer Moscovich, David H. Laidlaw, and Joseph J. LaViola, Jr. Cavepainting: A fully immersive 3d artistic medium and interactive experience. In Proceedings of the 2001 Symposium on Interactive 3D Graphics, I3D '01, pages 85-93, New York, NY, USA, 2001. ACM.

[53] Mohammadreza Khalilbeigi, Roman Lissermann, Wolfgang Kleine, and Jurgen Steimle. Foldme: Interacting with double-sided foldable displays. In Proceedings of the Sixth International Conference on Tangible, Embedded and Embodied Interaction, TEI '12, pages 33-40, New York, NY, USA, 2012. ACM.

[54] Hyunyoung Kim, Céline Coutrix, and Anne Roudaut. Knobslider: Design of a shape-changing device grounded in users' needs. In Actes De La 28IÈMe ConfÉRence Francophone Sur L'Interaction Homme-Machine, IHM '16, pages 91-102, New York, NY, USA, 2016. ACM.

[55] Seoktae Kim, Hyunjung Kim, Boram Lee, Tek-Jin Nam, and Woohun Lee. Inflatable mouse: Volume-adjustable mouse with air-pressure-sensitive input and haptic feedback. In Proceedings of the SIGCHI Conference on Human Factors in Computing Systems, CHI '08, pages 211-224, New York, NY, USA, 2008. ACM.

[56] David Kirk, Abigail Sellen, Stuart Taylor, Nicolas Villar, and Shahram Izadi. Putting the physical into the digital: Issues in designing hybrid interactive surfaces. In Proceedings of the 23rd British HCI Group Annual Conference on People and Computers: Celebrating People and Technology, BCS-HCI '09, pages 35-44, Swinton, UK, UK, 2009. British Computer Society.

[57] J. Knapp, J. Zeratsky, and B. Kowitz. Sprint: How to Solve Big Problems and Test New Ideas in Just Five Days. Simon \& Schuster, 2016. 
[58] Mami Kosaka and Kaori Fujinami. Unicrepaint: Digital painting through physical objects for unique creative experiences. In Proceedings of the TEI '16: Tenth International Conference on Tangible, Embedded, and Embodied Interaction, TEI'16, pages 475-481, New York, NY, USA, 2016. ACM.

[59] Matthijs Kwak and Joep W. Frens. From movement to mechanism: Exploring expressive movement qualities in shape-change. In Proceedings of the Ninth International Conference on Tangible, Embedded, and Embodied Interaction, TEI '15, pages 461-464, New York, NY, USA, 2015. ACM.

[60] Gierad Laput, Eric Brockmeyer, Scott E. Hudson, and Chris Harrison. Acoustruments: Passive, acoustically-driven, interactive controls for handheld devices. In Proceedings of the 33rd Annual ACM Conference on Human Factors in Computing Systems, CHI '15, pages 2161-2170, New York, NY, USA, 2015. ACM.

[61] Jonathan Lazar, Jinjuan Heidi Feng, and Harry Hochheiser. Research methods in human-computer interaction. Morgan Kaufmann, 2017.

[62] Mathieu Le Goc, Lawrence H. Kim, Ali Parsaei, Jean-Daniel Fekete, Pierre Dragicevic, and Sean Follmer. Zooids: Building blocks for swarm user interfaces. In Proceedings of the 29th Annual Symposium on User Interface Software and Technology, UIST '16, pages 97-109, New York, NY, USA, 2016. ACM.

[63] Bhoram Lee, Hyunjeong Lee, Soo-Chul Lim, Hyungkew Lee, Seungju Han, and Joonah Park. Evaluation of human tangential force input performance. In Proceedings of the SIGCHI Conference on Human Factors in Computing Systems, CHI '12, pages 3121-3130, New York, NY, USA, 2012. ACM.

[64] Daniel Leithinger, Sean Follmer, Alex Olwal, and Hiroshi Ishii. Physical telepresence: Shape capture and display for embodied, computer-mediated remote collaboration. In Proceedings of the 27th Annual ACM Symposium 
on User Interface Software and Technology, UIST '14, pages 461-470, New York, NY, USA, 2014. ACM.

[65] Daniel Leithinger and Hiroshi Ishii. Relief: A scalable actuated shape display. In Proceedings of the Fourth International Conference on Tangible, Embedded, and Embodied Interaction, TEI '10, pages 221-222, New York, NY, USA, 2010. ACM.

[66] Xin Liu and Jiawei Gu. Flexstroke: A flexible, deformable brush-tip with dynamic stiffness for digital input. In Proceedings of the 8th International Conference on Tangible, Embedded and Embodied Interaction, TEI '14, pages 39-40, New York, NY, USA, 2013. ACM.

[67] Andrés Lucero. Framing, aligning, paradoxing, abstracting, and directing: How design mood boards work. In Proceedings of the Designing Interactive Systems Conference, DIS '12, pages 438-447, New York, NY, USA, 2012. ACM.

[68] Andrés Lucero. Funky-design-spaces: Interactive environments for creativity inspired by observing designers making mood boards. In Julio Abascal, Simone Barbosa, Mirko Fetter, Tom Gross, Philippe Palanque, and Marco Winckler, editors, Human-Computer Interaction - INTERACT 2015, pages 474-492, Cham, 2015. Springer International Publishing.

[69] David Merrill, Emily Sun, and Jeevan Kalanithi. Sifteo cubes. In CHI ' 12 Extended Abstracts on Human Factors in Computing Systems, CHI EA '12, pages 1015-1018, New York, NY, USA, 2012. ACM.

[70] Timothy Robert Merritt, Mie Nørgaard, Christian Laursen, Majken Kirkegård Rasmussen, and Marianne Graves Petersen. Imagined physics: Exploring examples of shape-changing interfaces. In Cognitive Robotics, pages 89-111. CRC Press, 2015. 
[71] G. Michelitsch, J. Williams, M. Osen, B. Jimenez, and S. Rapp. Haptic chameleon: A new concept of shape-changing user interface controls with force feedback. In CHI 'O4 Extended Abstracts on Human Factors in Computing Systems, CHI EA ’04, pages 1305-1308, New York, NY, USA, 2004. ACM.

[72] Margaret R. Minsky. Manipulating simulated objects with real-world gestures using a force and position sensitive screen. SIGGRAPH Comput. Graph., 18(3):195-203, 1984.

[73] Viktor Miruchna, Robert Walter, David Lindlbauer, Maren Lehmann, Regine von Klitzing, and Jorg Muller. Geltouch: Localized tactile feedback through thin, programmable gel. In Proceedings of the 28th Annual ACM Symposium on User Interface Software \&\#38; Technology, UIST '15, pages 3-10, New York, NY, USA, 2015. ACM.

[74] Tamotsu Murakami, Kazuhiko Hayashi, Kazuhiro Oikawa, and Naomasa Nakajima. Do-it: Deformable objects as input tools. In Conference Companion on Human Factors in Computing Systems, CHI '95, pages 87-88, New York, NY, USA, 1995. ACM.

[75] Ken Nakagaki, Artem Dementyev, Sean Follmer, Joseph A. Paradiso, and Hiroshi Ishii. Chainform: A linear integrated modular hardware system for shape changing interfaces. In Proceedings of the 29th Annual Symposium on User Interface Software and Technology, UIST '16, pages 87-96, New York, NY, USA, 2016. ACM.

[76] Ken Nakagaki, Luke Vink, Jared Counts, Daniel Windham, Daniel Leithinger, Sean Follmer, and Hiroshi Ishii. Materiable: Rendering dynamic material properties in response to direct physical touch with shape changing interfaces. In Proceedings of the 2016 CHI Conference on Human Factors in Computing Systems, CHI '16, pages 2764-2772, New York, NY, USA, 
2016. ACM.

[77] Yusuke Nakagawa, Akiya Kamimura, and Yoichiro Kawaguchi. Mimictile: A variable stiffness deformable user interface for mobile devices. In Proceedings of the SIGCHI Conference on Human Factors in Computing Systems, CHI '12, pages 745-748, New York, NY, USA, 2012. ACM.

[78] Ryuma Niiyama, Xu Sun, Lining Yao, Hiroshi Ishii, Daniela Rus, and Sangbae Kim. Sticky actuator: Free-form planar actuators for animated objects. In Proceedings of the Ninth International Conference on Tangible, Embedded, and Embodied Interaction, TEI '15, pages 77-84, New York, NY, USA, 2015. ACM.

[79] Mie Nørgaard, Tim Merritt, Majken Kirkegaard Rasmussen, and Marianne Graves Petersen. Exploring the design space of shape-changing objects: Imagined physics. In Proceedings of the 6th International Conference on Designing Pleasurable Products and Interfaces, DPPI '13, pages 251260, New York, NY, USA, 2013. ACM.

[80] Masaru Ohkubo, Shuhei Umezu, and Takuya Nojima. Come alive! augmented mobile interaction with smart hair. In Proceedings of the 7th Augmented Human International Conference 2016, AH '16, pages 32:1-32:4, New York, NY, USA, 2016. ACM.

[81] Simon Olberding, Michael Wessely, and Jurgen Steimle. Printscreen: Fabricating highly customizable thin-film touch-displays. In Proceedings of the 27th Annual ACM Symposium on User Interface Software and Technology, UIST'14, pages 281-290, New York, NY, USA, 2014. ACM.

[82] Themis Omirou, Asier Marzo, Sue Ann Seah, and Sriram Subramanian. Levipath: Modular acoustic levitation for 3d path visualisations. In Proceedings of the 33rd Annual ACM Conference on Human Factors in Computing Systems, CHI '15, pages 309-312, New York, NY, USA, 2015. ACM. 
[83] Mai Otsuki, Kenji Sugihara, Asako Kimura, Fumihisa Shibata, and Hideyuki Tamura. Mai painting brush: An interactive device that realizes the feeling of real painting. In Proceedings of the 23nd Annual ACM Symposium on User Interface Software and Technology, UIST '10, pages 97-100, New York, NY, USA, 2010. ACM.

[84] Jifei Ou, Mélina Skouras, Nikolaos Vlavianos, Felix Heibeck, Chin-Yi Cheng, Jannik Peters, and Hiroshi Ishii. aeromorph - heat-sealing inflatable shape-change materials for interaction design. In Proceedings of the 29th Annual Symposium on User Interface Software and Technology, UIST '16, pages 121-132, New York, NY, USA, 2016. ACM.

[85] Hanbit Park and Minsoo Hahn. Mix brush: An interactive digital painting tool with shaking interaction and color feedback. In 3rd International Conference on Information Sciences and Interaction Sciences (ICIS), pages 46-49. IEEE, 2010.

[86] Joohee Park, Young-Woo Park, and Tek-Jin Nam. Wrigglo: Shape-changing peripheral for interpersonal mobile communication. In $\mathrm{CHI}$ ' 14 Extended Abstracts on Human Factors in Computing Systems, CHI EA '14, pages 189190, New York, NY, USA, 2014. ACM.

[87] Young-Woo Park, Joohee Park, and Tek-Jin Nam. The trial of bendi in a coffeehouse: Use of a shape-changing device for a tactile-visual phone conversation. In Proceedings of the 33rd Annual ACM Conference on Human Factors in Computing Systems, CHI '15, pages 2181-2190, New York, NY, USA, 2015. ACM.

[88] Amanda Parkes and Hiroshi Ishii. Bosu: a physical programmable design tool for transformability with soft mechanics. In Proceedings of the 8th ACM Conference on Designing Interactive Systems, pages 189-198. ACM, 2010. 
[89] Esben W. Pedersen, Sriram Subramanian, and Kasper Hornbæk. Is my phone alive?: A large-scale study of shape change in handheld devices using videos. In Proceedings of the SIGCHI Conference on Human Factors in Computing Systems, CHI '14, pages 2579-2588, New York, NY, USA, 2014. ACM.

[90] Ivan Poupyrev and Shigeaki Maruyama. Tactile interfaces for small touch screens. In Proceedings of the 16th Annual ACM Symposium on User Interface Software and Technology, UIST ’03, pages 217-220, New York, NY, USA, 2003. ACM.

[91] Ivan Poupyrev, Tatsushi Nashida, Shigeaki Maruyama, Jun Rekimoto, and Yasufumi Yamaji. Lumen: Interactive visual and shape display for calm computing. In ACM SIGGRAPH 2004 Emerging Technologies, SIGGRAPH '04, pages 17-, New York, NY, USA, 2004. ACM.

[92] Ivan Poupyrev, Tatsushi Nashida, and Makoto Okabe. Actuation and tangible user interfaces: The vaucanson duck, robots, and shape displays. In Proceedings of the 1st International Conference on Tangible and Embedded Interaction, TEI '07, pages 205-212, New York, NY, USA, 2007. ACM.

[93] Majken K. Rasmussen, Esben W. Pedersen, Marianne G. Petersen, and Kasper Hornbæk. Shape-changing interfaces: A review of the design space and open research questions. In Proceedings of the SIGCHI Conference on Human Factors in Computing Systems, CHI '12, pages 735-744, New York, NY, USA, 2012. ACM.

[94] Christian Rendl, David Kim, Patrick Parzer, Sean Fanello, Martin Zirkl, Gregor Scheipl, Michael Haller, and Shahram Izadi. Flexcase: Enhancing mobile interaction with a flexible sensing and display cover. In Proceedings of the 2016 CHI Conference on Human Factors in Computing Systems, CHI '16, pages 5138-5150, New York, NY, USA, 2016. ACM. 
[95] Simon Robinson, Céline Coutrix, Jennifer Pearson, Juan Rosso, Matheus Fernandes Torquato, Laurence Nigay, and Matt Jones. Emergeables: Deformable displays for continuous eyes-free mobile interaction. In CHI'16: 2016 CHI Conference on Human Factors in Computing Systems Proceedings, New York, NY, USA, 2016. ACM.

[96] Ilya Rosenberg and Ken Perlin. The unmousepad: An interpolating multitouch force-sensing input pad. ACM Trans. Graph., 28(3):65:1-65:9, 2009.

[97] Juan Rosso, Céline Coutrix, Matt Jones, and Laurence Nigay. Impact of mobile tangible slider design and its reachability on pointing performance. In Actes De La 28IÈMe ConfÉRence Francophone Sur L'Interaction HommeMachine, IHM '16, pages 70-78, New York, NY, USA, 2016. ACM.

[98] Juan Rosso, Céline Coutrix, Matt Jones, and Laurence Nigay. Simulating an extendable tangible slider for eyes-free one-handed interaction on mobile devices. In Proceedings of the 2018 International Conference on Advanced Visual Interfaces, AVI '18, pages 16:1-16:9, New York, NY, USA, 2018. ACM.

[99] Anne Roudaut, Abhijit Karnik, Markus Lochtefeld, and Sriram Subramanian. Morphees: Toward high shape resolution in self-actuated flexible mobile devices. In Proceedings of the SIGCHI Conference on Human Factors in Computing Systems, CHI '13, pages 593-602, New York, NY, USA, 2013. ACM.

[100] Anne Roudaut, Diana Krusteva, Mike McCoy, Abhijit Karnik, Karthik Ramani, and Sriram Subramanian. Cubimorph: designing modular interactive devices. In 2016 IEEE International Conference on Robotics and Automation (ICRA), pages 3339-3345. IEEE, 2016.

[101] Kimiko Ryokai, Stefan Marti, and Hiroshi Ishii. I/o brush: Beyond static collages. In CHI '07 Extended Abstracts on Human Factors in Computing 
Systems, CHI EA '07, pages 1995-2000, New York, NY, USA, 2007. ACM.

[102] Deepak Ranjan Sahoo, Kasper Hornbæk, and Sriram Subramanian. Tablehop: An actuated fabric display using transparent electrodes. In Proceedings of the 2016 CHI Conference on Human Factors in Computing Systems, CHI '16, pages 3767-3780, New York, NY, USA, 2016. ACM.

[103] Deepak Ranjan Sahoo, Timothy Neate, Yutaka Tokuda, Jennifer Pearson, Simon Robinson, Sriram Subramanian, and Matt Jones. Tangible drops: A visio-tactile display using actuated liquid-metal droplets. In Proceedings of the 2018 CHI Conference on Human Factors in Computing Systems, CHI '18, pages 177:1-177:14, New York, NY, USA, 2018. ACM.

[104] Jurgen Scheible and Timo Ojala. Mobispray: Mobile phone as virtual spray can for painting big anytime anywhere on anything. In ACM SIGGRAPH 2009 Art Gallery, SIGGRAPH ’09, pages 5:1-5:10, New York, NY, USA, 2009. ACM.

[105] Carsten Schwesig, Ivan Poupyrev, and Eijiro Mori. Gummi: A bendable computer. In Proceedings of the SIGCHI Conference on Human Factors in Computing Systems, CHI '04, pages 263-270, New York, NY, USA, 2004. ACM.

[106] Marcos Serrano, Anne Roudaut, and Pourang Irani. Investigating text legibility on non-rectangular displays. In Proceedings of the 2016 CHI Conference on Human Factors in Computing Systems, CHI '16, pages 498-508, New York, NY, USA, 2016. ACM.

[107] Orit Shaer, Eva Hornecker, et al. Tangible user interfaces: past, present, and future directions. Foundations and Trends® in Human-Computer Interaction, 3(1-2):4-137, 2010.

[108] Ben Shneiderman. Creativity support tools: Accelerating discovery and innovation. Commun. ACM, 50(12):20-32, December 2007. 
[109] Cameron Steer, Jennifer Pearson, Simon Robinson, and Matt Jones. Deformable paint palette: Actuated force controls for digital painting. In Proceedings of the 2017 CHI Conference Extended Abstracts on Human Factors in Computing Systems, CHI EA '17, pages 2936-2943, New York, NY, USA, 2017. ACM.

[110] Cameron Steer, Simon Robinson, Jen Pearson, Deepak Sahoo, Ian Mabbett, and Matt Jones. A liquid tangible display for mobile colour mixing. In To appear in the proceedings of the 20th International Conference on HumanComputer Interaction with Mobile Devices and Services, New York, NY, USA, 2018. ACM.

[111] Paul Strohmeier, Jesse Burstyn, Juan Pablo Carrascal, Vincent Levesque, and Roel Vertegaal. Reflex: A flexible smartphone with active haptic feedback for bend input. In Proceedings of the TEI '16: Tenth International Conference on Tangible, Embedded, and Embodied Interaction, TEI '16, pages 185-192, New York, NY, USA, 2016. ACM.

[112] Miriam Sturdee and Jason Alexander. Analysis and classification of shapechanging interfaces for design and application-based research. ACM Computing Surveys (CSUR), 51(1):2, 2018.

[113] Miriam Sturdee, Paul Coulton, and Jason Alexander. Using design fiction to inform shape-changing interface design and use. The Design Journal, 20(sup1):S4146-S4157, 2017.

[114] Miriam Sturdee, John Hardy, Nick Dunn, and Jason Alexander. A public ideation of shape-changing applications. In Proceedings of the 2015 International Conference on Interactive Tabletops \& Surfaces, pages 219-228. ACM, 2015.

[115] Kenji Sugihara, Mai Otsuki, Asako Kimura, Fumihisa Shibata, and Hideyuki Tamura. Mai painting brush++: Augmenting the feeling of painting with new 
visual and tactile feedback mechanisms. In Proceedings of the 24th Annual ACM Symposium Adjunct on User Interface Software and Technology, UIST '11 Adjunct, pages 13-14, New York, NY, USA, 2011. ACM.

[116] Ivan E. Sutherland. Sketch pad a man-machine graphical communication system. In Proceedings of the SHARE Design Automation Workshop, DAC '64, pages 6.329-6.346, New York, NY, USA, 1964. ACM.

[117] Faisal Taher, Jason Alexander, John Hardy, and Eduardo Velloso. An empirical characterization of touch-gesture input-force on mobile devices. In Proceedings of the Ninth ACM International Conference on Interactive Tabletops and Surfaces, ITS '14, pages 195-204, New York, NY, USA, 2014. ACM.

[118] Faisal Taher, John Hardy, Abhijit Karnik, Christian Weichel, Yvonne Jansen, Kasper Hornbæk, and Jason Alexander. Exploring interactions with physically dynamic bar charts. In Proceedings of the 33rd Annual ACM Conference on Human Factors in Computing Systems, CHI '15, pages 3237-3246, New York, NY, USA, 2015. ACM.

[119] Faisal Taher, Yvonne Jansen, Jonathan Woodruff, John Hardy, Kasper Hornbæk, and Jason Alexander. Investigating the use of a dynamic physical bar chart for data exploration and presentation. IEEE transactions on visualization and computer graphics, 23(1):451-460, 2017.

[120] Faisal Taher, John Vidler, and Jason Alexander. A characterization of actuation techniques for generating movement in shape-changing interfaces. International Journal of Human-Computer Interaction, 33(5):385-398, 2017.

[121] Haodan Tan, John Tiab, Selma Šabanović, and Kasper Hornbæk. Happy moves, sad grooves: Using theories of biological motion and affect to design shape-changing interfaces. In Proceedings of the 2016 ACM Conference on Designing Interactive Systems, pages 1282-1293. ACM, 2016. 
[122] Atau Tanaka and Adam Parkinson. Haptic wave: A cross-modal interface for visually impaired audio producers. In Proceedings of the 2016 CHI Conference on Human Factors in Computing Systems, CHI '16, pages 2150-2161, New York, NY, USA, 2016. ACM.

[123] Seppe Terryn, Joost Brancart, Dirk Lefeber, Guy Van Assche, and Bram Vanderborght. Self-healing soft pneumatic robots. Sci. Robot., 2(9), 2017.

[124] John Tiab, Sebastian Boring, Paul Strohmeier, Anders Markussen, Jason Alexander, and Kasper Hornbæk. Tiltstacks: Composing shape-changing interfaces using tilting and stacking of modules. In Proceedings of the 2018 International Conference on Advanced Visual Interfaces, AVI '18, pages 44:144:5, New York, NY, USA, 2018. ACM.

[125] John Tiab and Kasper Hornbæk. Understanding affordance, system state, and feedback in shape-changing buttons. In Proceedings of the $2016 \mathrm{CHI}$ Conference on Human Factors in Computing Systems, CHI '16, pages 2752 2763, New York, NY, USA, 2016. ACM.

[126] Garreth W. Tigwell, David R. Flatla, and Neil D. Archibald. Ace: A colour palette design tool for balancing aesthetics and accessibility. ACM Trans. Access. Comput., 9(2):5:1-5:32, 2017.

[127] Giovanni Maria Troiano, Esben Warming Pedersen, and Kasper Hornbæk. User-defined gestures for elastic, deformable displays. In Proceedings of the 2014 international working conference on advanced visual interfaces, pages 1-8. ACM, 2014.

[128] Theophanis Tsandilas, Magdalini Grammatikou, and Stéphane Huot. Bricosketch: Mixing paper and computer drawing tools in professional illustration. In Proceedings of the 2015 International Conference on Interactive Tabletops \& Surfaces, ITS '15, pages 127-136, New York, NY, USA, 2015. ACM. 
[129] Jessica Tsimeris, Colin Dedman, Michael Broughton, and Tom Gedeon. Forceform: A dynamically deformable interactive surface. In Proceedings of the 2013 ACM International Conference on Interactive Tabletops and Surfaces, ITS '13, pages 175-178, New York, NY, USA, 2013. ACM.

[130] Jessica Tsimeris, Duncan Stevenson, Tom Gedeon, and Matt Adcock. Using forceform, a dynamically deformable interactive surface, for palpation simulation in medical scenarios. In Proceedings of the Second International Workshop on Smart Material Interfaces: Another Step to a Material Future, SMI '13, pages 19-22, New York, NY, USA, 2013. ACM.

[131] Philip Tuddenham, David Kirk, and Shahram Izadi. Graspables revisited: multi-touch vs. tangible input for tabletop displays in acquisition and manipulation tasks. In Proceedings of the SIGCHI Conference on Human Factors in Computing Systems, pages 2223-2232. ACM, 2010.

[132] Peter Vandoren, Luc Claesen, Tom Van Laerhoven, Johannes Taelman, Chris Raymaekers, Eddy Flerackers, and Frank Van Reeth. Fluidpaint: An interactive digital painting system using real wet brushes. In Proceedings of the ACM International Conference on Interactive Tabletops and Surfaces, ITS '09, pages 53-56, New York, NY, USA, 2009. ACM.

[133] Peter Vandoren, Tom Van Laerhoven, Luc Claesen, Johannes Taelman, Fabian Di Fiore, Frank Van Reeth, and Eddy Flerackers. Dip - it: Digital infrared painting on an interactive table. In CHI '08 Extended Abstracts on Human Factors in Computing Systems, CHI EA ’08, pages 2901-2906, New York, NY, USA, 2008. ACM.

[134] Marynel Vázquez, Eric Brockmeyer, Ruta Desai, Chris Harrison, and Scott E. Hudson. 3d printing pneumatic device controls with variable activation force capabilities. In Proceedings of the 33rd Annual ACM Conference on Human Factors in Computing Systems, CHI '15, pages 1295-1304, New 
York, NY, USA, 2015. ACM.

[135] Luke Vink, Viirj Kan, Ken Nakagaki, Daniel Leithinger, Sean Follmer, Philipp Schoessler, Amit Zoran, and Hiroshi Ishii. Transform as adaptive and dynamic furniture. In Proceedings of the 33rd Annual ACM Conference Extended Abstracts on Human Factors in Computing Systems, CHI EA '15, pages 183-183, New York, NY, USA, 2015. ACM.

[136] Chihiro Watanabe, Alvaro Cassinelli, Yoshihiro Watanabe, and Masatoshi Ishikawa. Generic method for crafting deformable interfaces to physically augment smartphones. In CHI '14 Extended Abstracts on Human Factors in Computing Systems, CHI EA '14, pages 1309-1314, New York, NY, USA, 2014. ACM.

[137] Christian Weichel, Jason Alexander, and John Hardy. Shape display shader language (sdsl): A new programming model for shape changing displays. In Proceedings of the 33rd Annual ACM Conference Extended Abstracts on Human Factors in Computing Systems, pages 1121-1126. ACM, 2015.

[138] Martin Weigel, Tong Lu, Gilles Bailly, Antti Oulasvirta, Carmel Majidi, and Jurgen Steimle. Iskin: flexible, stretchable and visually customizable onbody touch sensors for mobile computing. In Proceedings of the 33rd Annual ACM Conference on Human Factors in Computing Systems, pages 29913000. ACM, 2015.

[139] Pierre Wellner. Interacting with paper on the digitaldesk. Commun. ACM, 36(7):87-96, 1993.

[140] John Williamson, Roderick Murray-Smith, and Stephen Hughes. Shoogle: Excitatory multimodal interaction on mobile devices. In Proceedings of the SIGCHI Conference on Human Factors in Computing Systems, CHI '07, pages 121-124, New York, NY, USA, 2007. ACM. 
[141] Micah Yairi. Optically clear film for tactile interfaces. In Society of PhotoOptical Instrumentation Engineers (SPIE) Conference Series, volume 9949, 2016.

[142] Lining Yao, Ryuma Niiyama, Jifei Ou, Sean Follmer, Clark Della Silva, and Hiroshi Ishii. Pneui: Pneumatically actuated soft composite materials for shape changing interfaces. In Proceedings of the 26th Annual ACM Symposium on User Interface Software and Technology, UIST '13, pages 13-22, New York, NY, USA, 2013. ACM.

[143] Neng-Hao Yu, Sung-Sheng Tsai, I-Chun Hsiao, Dian-Je Tsai, Meng-Han Lee, Mike Y. Chen, and Yi-Ping Hung. Clip-on gadgets: Expanding multitouch interaction area with unpowered tactile controls. In Proceedings of the 24th Annual ACM Symposium on User Interface Software and Technology, UIST'11, pages 367-372, New York, NY, USA, 2011. ACM.

[144] Oren Zuckerman and Ayelet Gal-Oz. To tui or not to tui: Evaluating performance and preference in tangible vs. graphical user interfaces. International Journal of Human-Computer Studies, 71(7-8):803-820, 2013. 